\title{
Matching mechanical cardiac support and the cardiovascular system
}

Citation for published version (APA):

Reesink, K. D. (2005). Matching mechanical cardiac support and the cardiovascular system. [Doctoral Thesis, Maastricht University]. Maastricht University. https://doi.org/10.26481/dis.20051213kr

Document status and date:

Published: 01/01/2005

DOI:

10.26481/dis.20051213kr

Document Version:

Publisher's PDF, also known as Version of record

\section{Please check the document version of this publication:}

- A submitted manuscript is the version of the article upon submission and before peer-review. There can be important differences between the submitted version and the official published version of record.

People interested in the research are advised to contact the author for the final version of the publication, or visit the DOI to the publisher's website.

- The final author version and the galley proof are versions of the publication after peer review.

- The final published version features the final layout of the paper including the volume, issue and page numbers.

Link to publication

\footnotetext{
General rights rights.

- You may freely distribute the URL identifying the publication in the public portal. please follow below link for the End User Agreement:

www.umlib.nl/taverne-license

Take down policy

If you believe that this document breaches copyright please contact us at:

repository@maastrichtuniversity.nl

providing details and we will investigate your claim.
}

Copyright and moral rights for the publications made accessible in the public portal are retained by the authors and/or other copyright owners and it is a condition of accessing publications that users recognise and abide by the legal requirements associated with these

- Users may download and print one copy of any publication from the public portal for the purpose of private study or research.

- You may not further distribute the material or use it for any profit-making activity or commercial gain

If the publication is distributed under the terms of Article $25 \mathrm{fa}$ of the Dutch Copyright Act, indicated by the "Taverne" license above, 


\section{Matching \\ Mechanical Cardiac Support and the Cardiovascular System}


2005 K.D. Reesink, Maastricht

ISBN 90-6464-225-7

Printed by: Grafisch Bedrijf Ponsen \& Looijen B.V., Wageningen

M.C. Escher's "Waterfall" $(2005$ The M.C. Escher Company B.V., Baarn, Holland. All rights reserved. 


\section{Matching \\ Mechanical Cardiac Support and the Cardiovascular System}

\section{PROEFSCHRIFT}

ter verkrijging van de graad van doctor aan de Universiteit Maastricht, op gezag van de Rector Magnificus, Prof, mr. G.P.M.F. Mols volgens het besluit van het College van Decanen, in het openbaar te verdedigen op dinsdag 13 december 2005 om 16.00 uur.

door

Koen Danï̈l Reesink 
Promotor:

Prof. dr. J.G. Maessen

\section{Co-promotor:}

Dr. ir. F.H. van der Veen

\section{Beoordelingscommissie:}

Prof. dr. A.P.M. Gorgels (voorzitter)

Prof, dr. is, M,G]. Arts

Prof. dr. B. Meyns (Katholieke Universiteit Leuven, B)

Prof. dr. MJ. Post

Prof. dr. ir. F.N. van de Vosse (Technische Universiteit Eindhoven)

Financial support by the Netherlands Heart Foundation, and the Stichting Hartsvrienden RESCAR for the publication of this thesis is gratefully acknowledged.

Additional support was granted by Arrow International. 
Leven is een opdracht van jezelf aan jezelf, Omwille van jezelf en dóór jezelf 


\section{Contents}

Chapter 1 General introduction 9

1.1 Heart failure 11

1.2 Mechanical support devices 13

1.3 Application of mechanical support 18

1.4 Measurement of physiologic function 22

1.5 On the contents 23

Chapter 2 Feasibility study of a fiber-optic system for invasive blood pressure measurements

Chapter 3 Synchronously counterpulsating extracorporeal life support enhances myocardial working conditions. regardless of systemic perfusion pressure

Chapter 4 The acute effect of intra-aortic balloon counterpulsation during extracorporeal life support

Chapter 5 Physiologic insensitive left ventricular assist predisposes right-sided circulatory failure: A pilot simulation and validation study

Chapter 6 Miniature intracardiac assist device provides more effective cardiac unloading and circulatory support during severe left heart failure than intra-aortic balloon pumping

Chapter 7 Circulatory instability due to left ventricular assist: device suction: Cause and remedy 
Chapter 8 General discussion

8.1 Heart failure: remodeling and reverse remodeling $\quad 158$

8.2 The young student 161

8.3 Hemodynamic collapse due to LVAD suction $\quad 162$

8.4 Optimization of mechanical support 164

8.5 Characteristics of the conceptual model 165

8.6 Comments 169

8.7 Future work 169

$\begin{array}{ll}8.8 \text { Conclusion } & 170\end{array}$

$\begin{array}{ll}\text { Summary } & 173\end{array}$

$\begin{array}{ll}\text { Samenvatting } & 175\end{array}$

$\begin{array}{ll}\text { Dankwoord } & 179\end{array}$

$\begin{array}{ll}\text { Curriculum Vitae } & 181\end{array}$

$\begin{array}{lr}\text { Publications } & 182\end{array}$ 
General introduction 19

\section{Chapter 1}

General introduction 
A student consults bis general practitioner. Four days earlier be felt be was devaloping flat, but presently be is short of breath and suffers froms extreme fatigute. Two days later matters are exen worse and the GP sends bine to a local bospital because of suspected beart failure. No clear diagnosis can be made, so be is transferred to an academic bospital. Upon presentation the young man is acute ill: vemperature is 39.7 degrees Celsius, beart rate is 160 beats per minute, blood pressure is $60 \mathrm{~mm} \mathrm{Hg}$, and be is panting beavily. The patient is catbeterized, diagnosed with acute beart failure, and put on inotropic support. The family and patient are being asked whether they would consent mechanical circulatory support or beart transplantation if necessary. They agree. The patient's benwodynawic condition worsens quickly despite maximum pharmacological support. The doctors decide to go for assist device implantation at once.

In the operating room, an emergency beart-lung macbine is inserted wia the patient's femoral vessels under local anesthesia. Later, under general anesthesic an artificial pumping chamber is implanted which pumps the blood from the lungs towards the aorta. The beart-lung machine can now be disconnected, because the left wentricular assist device (LVAD) provides sufficient blood flow. Laboratory analyses provide no clear cause for the young man's pump failure. The patient is referred to the cardiac intensive care unit.

Eleven days after the operation (POD 11) the contractile function of the beart is assessed by $2 D$ imaging: left ventricular ejection fraction is only 14\%. After sixteen days, no improvement at all is seen. Unfortwnately, no donor hean has yet become available. On the thirty-ninth $P O D$, ejection fraction bas improved up to $28 \%$. The doctors decide to pull the patient from the transplantation waiting list. Progressively, cardiac function innproves and on POD 67 the patient shows a completely normalized hemodynamic status without any pharmacological or mechanical support. Three days later the device is explanted. Ten days after device removal the patient returms bome and after wo months rebabilitation the student is able to resume bis studies at the wiversity'. 
The clinical case described above had a favotable outcome, which is being ascribed to the use of mechanical circulatory support (MCS) and its properties. A number of issues should be looked at more closely.

Firstly, an assist device was employed to cope with the critical condition of the patient and to buy time until a donor heart would become available (bridging to transplantation). However, mechanical support had a beneficial effect on recovery, so ultimately, the patient was bridged to recovery. Secondly, the cause of heart failure was unknown before mechanical support was instituted. The indication for assist device use appears to be difficult to be defined. Thirdly, during the extended period of support, the reserve capacity of the native heart was assessed by a comprehensive monitoring protocol. These measurements provided the necessary information on the patient's changing cardiac function, and thus enabled explantation of the assist device.

In the following three paragraphs, the use of mechanical circulatory support in heart failure patients is given a background by addressing who is in need of support, which devices are available, and how support is applied.

\subsection{Heart failure}

The most common forms of heart failure in western society are ischemic and idiopathic cardiomyopathy ${ }^{1-6}$. In ischemic disease the function of the myocardium is impaired by an imbalance in oxygen supply and demand, while the latter term describes heart failure of unknown etiology 7.8 . Mechanical problems such as valvular insufficiency and septal defects may directly cause pump failure, but more often they induce heart failure because their existence requires extraordinary adaptations from the heart and circulatory system ${ }^{8.11}$. The process by which the heart adapts to thythmic, metabolic, or mechanical demands is called cardiac remodeling. Essentially, adaptation as such is a desirable process as it copes with both systemic and environmental changes ${ }^{12-17}$.

As long as a failing heart can sustain life, we speak of compensatory heart failure. Decompensation starts when the heart's pumping capacity declines further and becomes life-threatening. In heart failure, further 
distinction is made between acute and chronic failure. The reversibility of the disease is the most important issue when therapy is considered. Unfortunately, acute and chronic failure are not straightforwardly associated with reversible and irteversible disease processes ${ }^{3,4,18-22}$.

\section{Ischemic beant disease}

Acute myocardial infarction and chronic coronary artery disease are the prevalent forms of ischemic heart disease. The contractile function of ischemic myocardium is decreased because of an imbalance in oxygen demand and supply. This loss of function may be due to present hypoperfusion, or may be due to past ischemic events. The latter suggests that the myocardium has become either necrotic, or that it has adapted its internal processes in order to avoid permanent destruction, which is called myocardial stunning and/or hibernation. Myocardium that is stunned or hibernating is considered viable tissue that may retain its contractile function, if conditions are created that enable endogenous or exogenous repair ${ }^{4,21}$.

\section{Treatment of beart failure}

Pharmacological therapy is the first option of treatment for the preservation of viable myocardium and the restoration of cardiac function. This approach appears successful in patients with minor to moderate heart failure ${ }^{3,42023}$, but in case of severe heart failure, a combination with the more aggressive measures provided by interventional cardiology and cardiac surgery is required. The number of treatment options offered by interventional cardiology is increasing rapidly. Percutaneous interventions (PCI) such as coronary angioplasty and coronary stenting are used routinely $4,20,23,24$. Cardiac surgical options include valve replacement, coronary artery bypass grafting, passive cardiac constraint implantation, and surgical ventricular restoration 16,25-28. For end-stage heart failure, cardiac transplantation has become the most promising solution. However, less than five percent of the transplant candidates can be treated, because the incidence of severe cardiovascular disease remains ever increasing and the amount of donor organs lag behind persistently ${ }^{18,29,30}$. 
When the cardiologist's approach remains insufficient and the patient is ineligible for surgical treatment or untesponsive to it, the only option left is mechanical circulatory support (MCS). MCS is a means to either assist the heart in its function or to take over the task of perfusing the body altogether. In this way, life is sustained and time is bought to enable intervention, whether it is pharmacological, minimally invasive, or surgical. MCS is a broad term covering the use and management of a wide range of pump systems (Table 1.1): from the heart-lung machine (HLM), which features maximum cardiopulmonary bypass, to the intra-aortic balloon pump (IABP), the least invasive cardiac assist pump ${ }^{24,31,32}$. In between these MCS extremes, the ventricular assist device (VAD) and extracorporeal life support (ECLS) circuit offer the intermediate options. In the last three decades, considerable technological and scientific progress has been made in this field. In the next paragraph the development and specific characteristics of these assist options will be reviewed.

\subsection{Mechanical support devices}

The first clinical use of mechanical circulatory support was reported in 1953, when Gibbon employed a cardiopulmonary bypass setup for the treatment of pulmonary embolism. Interestingly, even before the first human heart transplantation had taken place, experience with artificial hearts in an experimental context had already been obtained. In 1957, both Kolff and Akutsu replaced the native heart of a dog with a total artificial heart (TAH); the animal managed to stay alive for 90 minutes. Ten years later, the first human heart transplantation took place. Apparently, this advancement encouraged surgeons with an artificial organ program to proceed with human cardiac replacement. Already in 1969, Cooley and his team implanted a TAH in a patient. The patient survived for 64 hours and died of multi organ failure. Governmental grant programs provided the financial backup for artificial heart research and development from the mid 60 s to the late 80 s. Encouraging results were obtained in 1982, when patient Barney Clark survived for 112 days on the Jarvik-7 total artificial heart ${ }^{2}$. Nonetheless, the total atrificial heart and the associated class of patients 


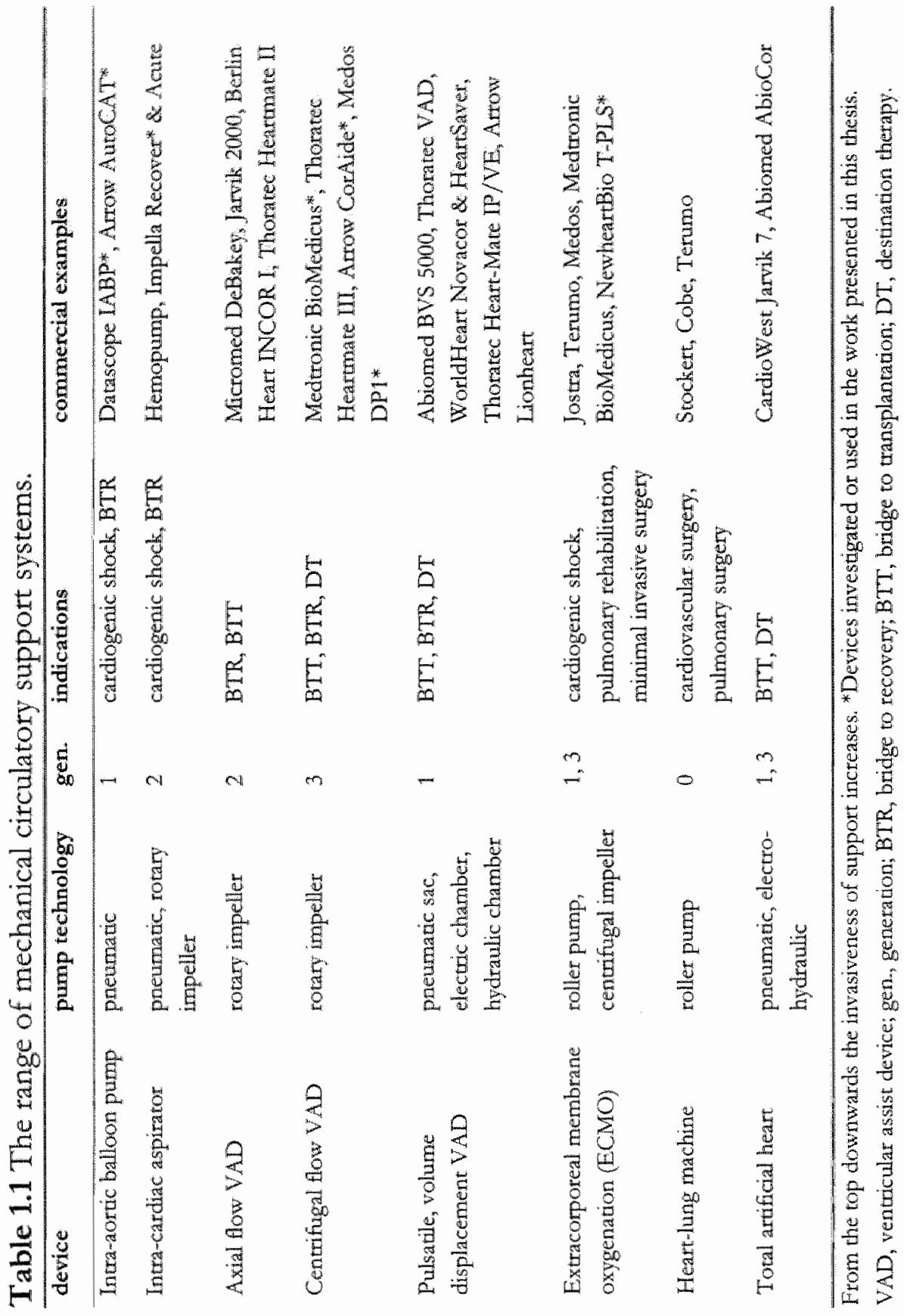


posed enormous problems both clinically and ethically. Therefore, the major funding programs were stopped in 1988 and were redirected towards the further development of the ventricular assist device (VAD) ${ }^{29.33}$. The progress in this field has ultimately led to the frequent use of several types of VAD in the 90s, and the official approval of some of the devices for the specific use as a bridging modality. Recently, the benefit of LVAD support over the medical treatment of end-stage heart failure patients NYHA class III/IV) was shown in the REMATCH trial ${ }^{2}$. Current developments lead towards the use of MCS as a destination therapy (DT) ${ }^{2,33-36}$.

\section{Technology}

The artificial hearts that have been developed over the last 40 years provide an interesting recapitulation of technological progress. The stringent safety and reliability requirements and the simultaneous necessity for flexibility, pose great challenges for the design of MCS. Table 1.1 summarizes the range of MCS systems available today in the order of their invasiveness. A chronological indication of the first design and clinical appearance is given by a generation tag for each device. Generation 'zero' (1950-60) relied on the technique of displacing blood volume in a tube by compression, which is still a clinical standard today (roller pump). The next generation (1960-80; gen. 1) is also comprised of volume displacement pumps, but these designs come closest to replicas of the natural heart. Later generations of blood pumps are optimized for efficiency, reliability, and longevity (1980-2000; gen. 2 and 3 ).

Historically, artificial hearts were designed to mimic the much valued pulsating action of the native heart. These devices employ a piston actuated pressure chamber which passively or actively fills with blood through an inflow valve, and ejects the same amount through an outflow valve ${ }^{18,31-33,37,38}$. This first generation of artificial hearts thus provided a pulsatile flow, at the expense of a fairly complex pump design. Some of these pump systems were and are still powered by robust drive units (Fig. 1.1). The mechanical life expectancy of these pumps was limited because of mechanical wear and biocompatibility problems such as valve leakage, thrombus formation, device pocket and driveline infections ${ }^{18,32,39,40}$. 


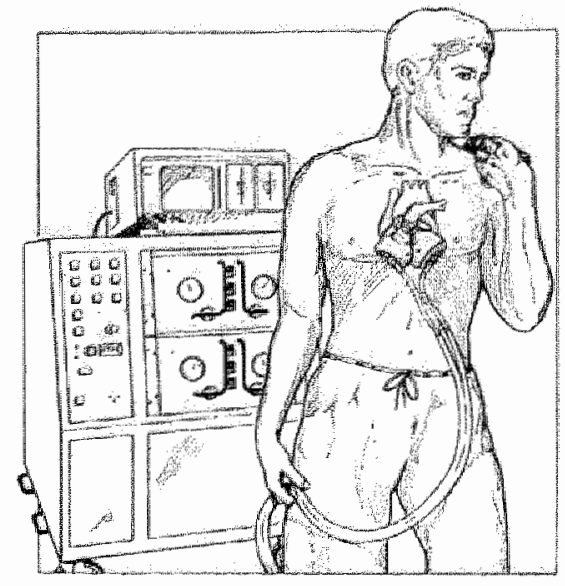

Figure 1.1 A Jarvik-7 total artificial heart (orig. 1970) recipient. The two chambers are driven from a pneumatic console via percutaneous drivelines. In early days safety and reliability requirements often called for a bullky and hardly mobille console.

Because the clinical applications and indications shifted gradually, pumps that were not capable of producing the entire cardiac output themselves gained interest. In stead of taking over the full cardiac capacity, the major goal of support was to assist the heart in its function. Therefore, the alleged necessity for pulsating pumps became less of an issue, and high-tech rotary fuel pump designs from aerospace engineering were adopted. These pumps are smaller, lighter, more reliable and cheaper than the first generation devices. Axial flow technology features high flows against relatively low opposing pressures. A radial (centrifugal) pump design enables the production of higher drive pressures but has therefore a limited flow capacity. In the latest rotary blood pump designs mechanical wear has been brought to a minimum by incorporating only one moving part and sophisticated bearing technologies such as magnetic levitation (Fig. 1.2) $14,32,35,36,38,41-46$ 


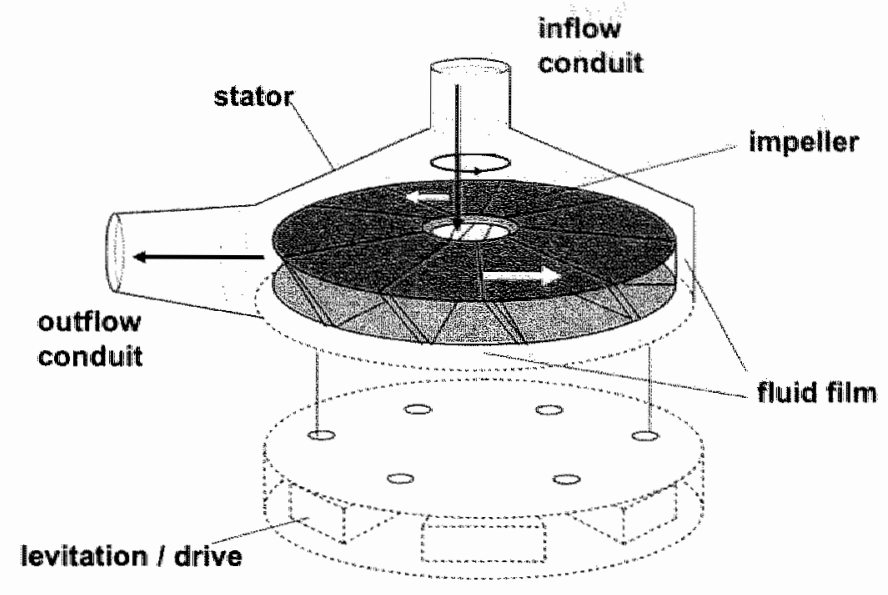

Figure 1.2 Illustration of the construction of a magnetically levitated centrifugal pump.

A crucial aspect of MCS development is the implementation of the power source. In the $70 \mathrm{~s}$, nuclear power was considered the ultimate solution to provide the patient untethered ambulatory mobility. In reality, the freedom of movement was limited, and psychological and safety issues halted the further development. Power transfer is less of an issue with the temporary use of assist devices, so bedridden or ambulatory hospitalized patients receive their power supply from pneumatic and electric drive units, via percutaneous tubes or cables (Fig. 1.3). With long term support, the drivelines have to be disposed of to reduce the susceptibility to infections. Inductive transcutaneous energy transfer (TET) is successfully applied in a number of commercial assist devices ${ }^{33,39,47}$. The control and monitoring signals towards and coming from the assist device can also be accommodated by wireless interfacing. The energy associated with the data transfer is small compared to the hydrodynamic power requirement of blood pumps which is in the order of 10 Watts.

Today, the functional interaction of mechanical support devices with the native circulation is incteasingly important, because 
biocompatibility problems such as thromboembolism, infection, and hemolysis have been addressed with considerable result in the last 30 years.

\subsection{Application of mechanical circulatory support}

Indications and limitations

As pointed out before, a broad range of indications for the use of MCS exist. Ventricular assist devices bridge patients to heart transplantation (BTI), to recovery (BTR), or they constitute a permanent option, which is termed destination therapy ${ }^{30,48}$.

With BTT use, not only time is bought till a donor heart becomes available, but the ineligibility for heart transplantation of patients with severe pulmonary hypertension may be reversed as well ${ }^{14,49,50}$. The

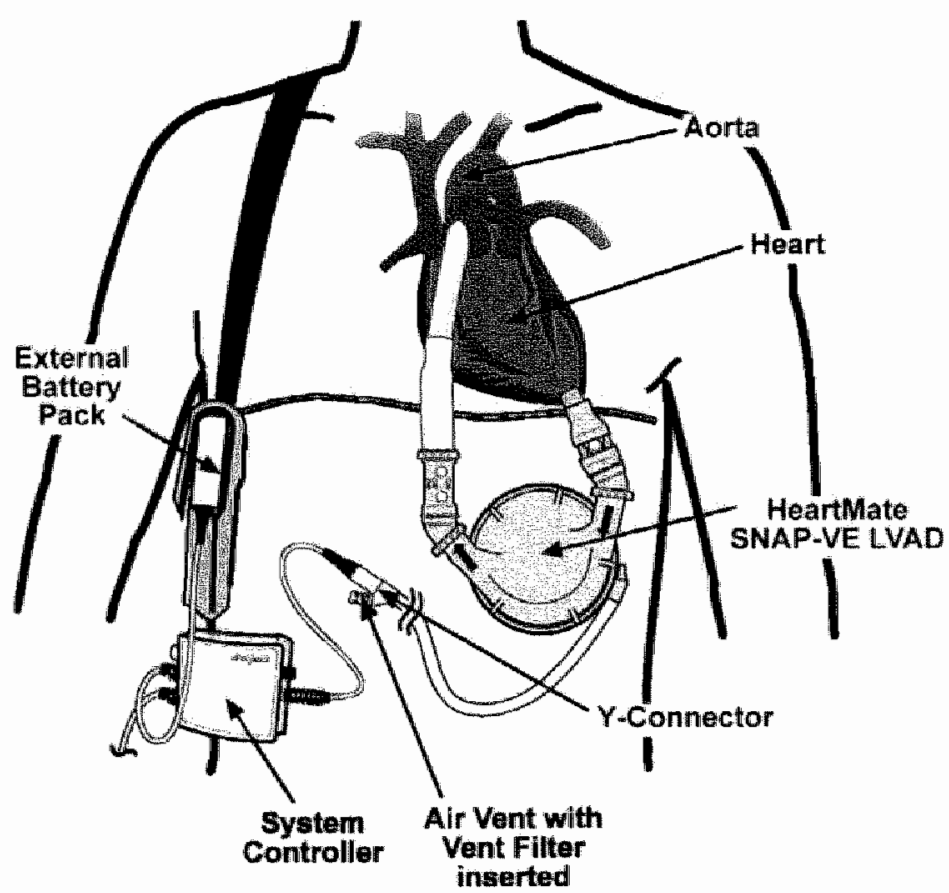

Figure 1.3 Typical arrangement of an implantable left ventricular assist device (LVAD) for ambulatory patients. The example shows the HeartMate I, an electrically driven pulsatile LVAD. 
continuous pulmonary venous unloading provided by a $L V A D$, appears effective in facilitating reverse remodeling of the stiffened pulmonary vasculat bed. In shock patients, the regression of acute pulmonary edema through left ventricular unloading breaks the vicious cycle which would otherwise lead to death quickly. The functional improvement of end-organs prior to transplant through the use of MCS has been shown to increase heart transplantation success rates $4,17,51,52$.

Initially, BTR use was not considered until encouraging experiences of cardiac recovery on the device were obtained $10,34,40,44$. In a number of LVAD recipients, device related complications, such as stroke and malfunction, urged premature discontinuation. Remarkably, some of these previously damaged hearts showed good and stable function after device explantation. Ventricular assist devices have been used for the treatment of acute myocarditis, dilated cardiomyopathy, and postcardiotomy heart failure $15,35,39,44,49,53$. Mechanical unloading therapy may require support periods of a week up to four years. However, the application of medium to long term support is extremely costly and subject to significant morbidity ${ }^{29,31,54}$.

Originally designed for the treatment of neonatal and pediatric pulmonary failure, extracorporeal membrane oxygenation (ECMO) has found its application in adult cardiopulmonary resuscitation as well. A number of expert centers have treated a considerable amount of patients successfully, but the mortality rates remain greater than 60 percent ${ }^{22,39,55,56}$. Compared to VAD support, adult ECMO, also termed extracorporeal life support (ECLS), is potentially more powerful. Severe pump failure, especially the longer standing, elicits the deterioration of other vital systemic functions such as the cerebral, renal, and hepatic. The extended cardiopulmonary bypass capacity of ECLS makes it suitable to prevent this development of multi-organ failure (MOF) which is mostly fatal. Inherently, ECLS is also the most complex form of MCS because of the multitude of aims associated with MOF and cardiopulmonary resuscitation. Due to its invasiveness a host of complications are involved such as bleeding, thromboembolism, infection, and hemolysis. Because of the abovementioned reasons ECLS is considered suitable for short to midterm bridging applications only $22,55,56$. 
From a pragmatic viewpoint, the use of MCS for tecovery purposes (BTR) is desirable but also challenging. The remainder of this thesis is dedicated to this aspect of mechanical support therapy.

\section{Adjustment and control}

The design and execution of a protocol for the management MCS of varies with each institution, and depends mainly on clinical experience ${ }^{34,39,40,55-57}$. In general, the support period may be subdivided into three stages: initiation, maintenance, and weaning. The initial period is often characterized by the application of maximum support: i.e. the assist device runs at full capacity within certain safety limits. Hereby, the heart and associated circulatory systems are fully unloaded to enable them to recuperate. In the next stage, support should be continued to maintain favorable conditions for recovery. The crux, however, is that the longer mechanical support is provided, the higher morbidity will be. On the other hand, premature discontinuation may limit efficacy and cause relapse. The weaning stage involves a similar conflict. When support is discontinued promptly, the heart has to respond accordingly but it may not be capable to do so because of its past inactivity due to unloading. Thus, a more gradual and well-controlled process of weaning is mandatory. The statement 'weaning starts the moment the pump is switched on' refers to the obvious desire to use the rigors of MCS only to the extent of necessity, and puts the monitoring of recovery forward as the crucial aspect of therapy.

Successful weaning strategies all rely on the frequent evaluation of cardiac function by e.g. echocardiography and pulmonary catheterization $15,34,3,40,55.58$. The first step in the assessment of native cardiac function, is a conservative increase in cardiac workload which is produced by either reducing the bypass flow or by dyssynchronizing the assist system ${ }^{40,57}$. If good results are obtained, cardiac loading is increased by prolonging the unsupported period. The next step involves mobilization of the patient and moderate exercise. The difference in exercise capacity with and without device support informs the clinician on the progress of recovery. When the patient has made sufficient progress, the device is explanted and a cardiac 
rehabilitation program is initiated $5,1 \%, 34$. Obviously, the markers for full recovery are manifold and not only include cardiopulmonary indices.

\section{Control engineering}

The insensitivity of mechanical blood pumps to physiological demand has been addressed by the control engineering society. Many different physiologic feedback control designs have been proposed to warrant the safety and efficacy of artificial hearts. The multitude of control objectives for total artificial heart (TAI) controllers has led to designs that incorporate an adaptive and model based topology ${ }^{59-62}$. The associated requirement for on-line system identification techniques has produced a substantial knowledge base for cardiovascular modeling, computer simulation, and experimental validation ${ }^{63.66}$. Unfortunately, scientific papers that detail the clinical performance of these control schemes are scarce ${ }^{59}$. With ventricular assist devices, the approach to control design has been more pragmatic. The characteristic property of the native ventricle, i.e. to adjust its output to preload, is designated as the primary control aim. A good preload response should automatically satisfy the required pump output and avoid problems with suction. Under pathological conditions, however, automatic adjustment could be ineffective or even dangerous without the expert judgment of the clinician. Various circulatory models have been developed for the study of device-circulation interactions and for the purpose of feedback control design ${ }^{67.71}$. The design and optimization of a physiologic control algorithm is an iterative process, which requires a flexible, durable, and reproducible testing environment. Animal testing and clinical evaluations are limited in this respect. Unfortunately, model validation studies are not abundant ${ }^{70}$.

\section{The necessity for information}

The decision to use MCS in a patient is a difficult one. First of all, the benefits should outweigh the drawbacks of support, but this is never a straightforward issue. There is little time left for discussion when a patient goes into severe shock, but when the first dangers have been averted, the question whether the patient will fully recuperate or not is pertinent. 
However, once life support has been statted, the decision to stop (or how to stop) may be difficult. The primary issue here is that of prognosis: If one can predict, from measurements, that a patient will or will not recover over time, the decision to maintain or discontinue life support may be less critical. One could argue that the heart of the weaning problem is the availability of information on which the decision is based. To stress the relevance of measuring physiologic function, a brief theoretical perspective is given below.

\subsection{Measurement of physiologic function}

Only a limited evaluation of cardiac function can be made in the assisted circulation because the heart is unloaded and performs little work. A change in external conditions is necessary to obtain more informative data. In Figure 1.4, such a change in demand is shown along the curve denoted by 'health'. When we compare the two linear relationships, it is evident that during illness, expense is greater than in the healthy condition for the same performance. With health efficiency is higher than during illness: A higher

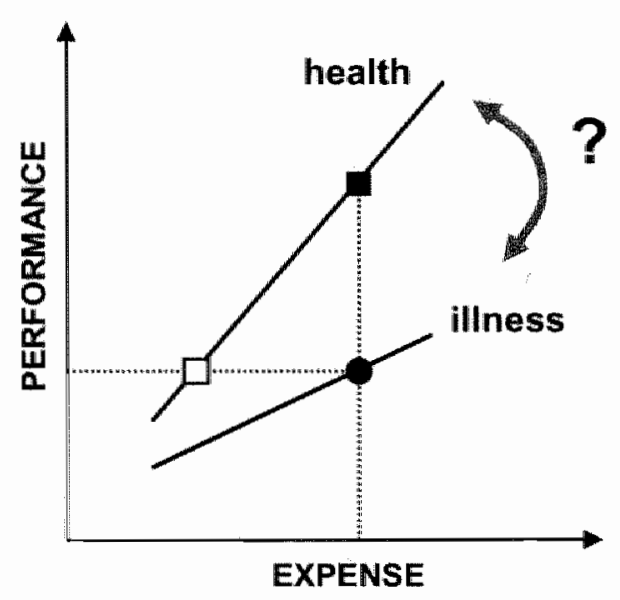

Figure 1.4 Graphical representation of health and illness translated in input-output relationships. The diagram illustrates the disadvantageous condition of illness in terms of performance level versus energy expense. 
performance can be obtained with a similar amount of consumed energy. The dynamic evaluation of two or more physiological variables enables the calculation of intrinsic properties such as cardiac contractility, reserve capacity, etc. In the example shown below, this intrinsic capacity is the amount of work performed per unit of consumed energy, which is an inputoutput relationship. During MCS, functional evaluations can be made by transiently disturbing pump speed or flow.

The linear interpretation of physiological measurement above, fails to address an important issue: the development of a disease, as well as the recovery process, is a continuous process. In Figure 1.4, however, a discontinuity exists between both linear curves. Furthermore, a causal relationship between loading and its role in the change of physiological function is missing ${ }^{12.72}$. In Chapter 8 , the graphical analysis is developed further and will be applied to the processes of heart failure development, cardiac remodeling and reverse remodeling, and therapy. The role of mechanical circulatory support as a bridge to recovery and the process of weaning will be addressed specifically.

\subsection{On the contents}

This thesis contains six papers that cover a range of aspects of mechanical circulatory support and hemodynamic measurement. The first paper (Chapter 2) describes the preclinical evaluation of a prototype pressure sensor developed for diagnostic and device triggering purposes. Chapters 3 and 4 address the functional interactions between an ECLS circuit and the native heart. In both papers the use of counterpulsation as a means to optimize cardiac conditions during support is evaluated. The last part of the thesis is dedicated to the interaction of a continuous flow LVAD with the left ventricle and the circulation in general. Chapter 5 describes the design and validation of a computer simulation model. In Chapter 6 an intracardiac axial flow device is compared to its clinical peer: the intra-aortic balloon pump. In the last paper, the effect of LVAD suction on the pulmonary circulation is investigated. The general discussion (Chapter 8) is dedicated to integrate the findings presented in the individual papers. 


\section{References}

1 Holman WL, Bourge RC, Kirklin JK. Case report: circulatory support for seventy drys with tesolution of acute heart failure. I Thorac Cardiovasc Surg 1991; $102: 932-934$

2 Rose EA, Gelins AC, Moskowitz AJ, et al. Long-Term Use of a Left Ventricular Assist Device for End-Stage Heart Failure. N Engl J Med 2001; 345:1435-1443

3 Fonatow GC. Managing the patient with diabetes mellitus and heart failure: issues and considerations. Am J Med 2004; 116 Suppl 5A:76S-88S

4 Hollenberg SM, Kavinsky CJ, Parrillo JE. Cardiogenic shock. Ann Intern Med $1999 ; 131: 47-59$

5 Pozehl B, Duncan K, Krueger $S$, et al. Adjunctive effects of exercise training in heart failure patients teceiving maximum pharmacologic therapy. Prog Cardiovasc Nurs 2003; 18:177-183

6 Ho KK, Pinsky JL, Kannel WB, et al. The epidemiology of heart failure: the Framingham Study. J Am Coll Cardiol 1993; 22:6A-13A

7 Abraham WT, Fisher WG, Smith $A L$, et al. Cardiac resynchronization in chronic heart failure. N Engl J Med 2002; 346:1845-1853

8 Lauer MS, Iarson MG, Evans JC, et al. Association of left ventricular dilatation and hypertrophy with chronotropic incompetence in the Framingham Heart Study. Am Heart J 1999; 137:903-909

$9 \quad$ Kaye DM, Byrne M, Alferness C, et al. Feasibility and short-term efficacy of percutaneous mitral annular reduction for the therapy of heart failure-induced mitral regurgitation. Circulation 2003; 108:1795-1797

10 Braun J, Bax JJ, Versteegh MI, et al. Preoperative left ventricular dimensions predict reverse remodeling following restrictive mitral annuloplasty in ischemic mitral regurgitation. Eur ] Cardiothorac Surg 2005; 27:847-853

11 Dekker AL, Barenbrug PJ, van der Veen FH, et al. Pressure-volume loops in patients with antic stenosis. J Heart Valve Dis 2003; 12:325 332

12 Bateson G. The tole of somatic change in evolution. Steps to an ecology of mind. New York: Ballantine Books, 2000; 533

13 Gordon NF, Gulanick M, Costa F, et al. Physical Acrivity and Exercise Recommendations for Stroke Survivors: An American Heart Association Scientific Statement From the Council on Clinical Cardiology, Subcommittee on Exercise, Cardiac Rehabilitation, and Prevention; the Council on Cardiovascular Nursing; the Council on Nutrition, Physical Activity, and Metabolism; and the Stroke Council. Circulation 2004; 109:2031-2041

14 Salzberg SP, Lachat ML, von Harbou K, et al. Normalization of high pulmonary vascular resistance with LVAD support in heart transplantation candidates. European Journal of Cardio-Thoracic Surgery 2005; 27:222-225 
15 Hetzer $\mathbb{R}$, Muller J, Weng $\mathrm{Y}$, et ai. Cardinc recovery in dilated cardiomyopathy by unloading with a left ventricular assist device. Ann Thorac Surg 1999; 68:742749

16 Dor V. Surgical remodeling of left ventricle Surg Clin North Am 2004; 84:27-43

17 Guyton A, Hall J, eds. Textbook of medical physiology. 9 ed. Philadelphia, $P$ A: W.B. Saunders Company; 1996

18 Procedings. of The fifth international conference on circulatory suppots dentices for severe heart failure. 2000, New York. In: Pennington D, ed. Supplement to "The Annals of Thonacic Surgery, Vol. 71 (Supplement 3), March. New York: Elsevier Science Inc, 2001; S55-222

19 Hetzer R, Muller $J H_{3}$ Weng $\mathrm{Y}_{\mathrm{mg}}$, et al. Bridging-tomrecovery. The Annals of Thoracic Surgery 2001; 71:S109-S113

20 Kloner RA, Rezkalla SH. Cardiac protection during acute myocardial infarction: Where do we stand in 2004? Joumal of the American College of Cardiology 2004; 44:276-286

21 Mangano DT. Myocardial stunning: an overwiew. J Card Surg 1993; 8:204-213

22 Pagani FD, Aaronson KD, Swaniker F, et al. The use of extracorporeal life support in adult patients with primary cardiac failure as a bridge to implantable left ventricular assist device. The Annals of Thonacic Surgery 2001; 71:\$77-\$81.

23 Giugliano RP, Sabatine MS, Gibson CM, et al. Combined assessment of thrombolysis in myocardial infarction tlow grade, myocardial perfusion grade, and ST-segment resolution to evaluate epicardial and myocardial reperfusion. Am J Cardiol 2004; 93:1362-1367, A1365-1366

24 Weber $K T^{*}$, Janicki JS. Inttaaortic balloon counterpulsation. A review of physiological principles, clinical results, and device safety. Ann Thorac Surg 1974; $17: 602-636$

25 Batista RJ, Santos JL, Takeshita N, et al. Partial left ventriculectomy to improve left ventricular function in end-stage heart discase. J Card Surg 1996; 11:96-97; discussion 98

26 Harada $H$, Honma $Y$, Hachiro $Y$, et al. Batista operation with aortic valve replacement for valvular cardiomyopathy. Ann Thorac Cardiowasc Surg 2003; 9:138-141

27 Schreuder J], Steendijk P, van der Veen FH, et al. Acure and short-term effects of partial left ventriculectomy in dilated cardiomyopathy: assessment by pressurevolume loops. J Am Coll Cardiol 2000; 36:2104-2114

28 Bainbridge D, Martin I, Cheng D. Off pump coronary artery bypass graft surgery versus conventional coronary artery bypass graft surgery: a systematic teview of the literature. Semin Cardiothorac Vase Anesth 2005; 9:105-111

29 Marshall E. A space age vision advances in the clinic. Science 2002; 295:10001001 
30 Helman DN, Rose EA. History of mechanical circulatory support. Prog Cardiovase Dis 2000; $43: 1-4$

31 Chait $\mathrm{H}$, Willams J. Mechanical circulatory suppott. Postoperative management of the cardiac surgical patient. see MSc thesis: $\mathrm{xxxx}, \mathrm{xxxx} ; 357-408$

32 Goldstein D, Oz M, eds. Cardiac assist devices. 1 ed. Amonk, NY: Futura Publishing, 2000

33 Dowling RD, Gray LA, Jr., Etoch SW, et al. The AbioCor implantable replacement heart. Ann Thorac Surg 2003; 75:\$93-99

34. Yacoub MH, Tansley $P$, Birks EJ, et al. A novel combination therapy to reverse end-stage heart failure. Transplant Proc 2001; 33:2762-2764

35 Westaby $S$, Banning $A P_{\text {\& }}$ Saito $S$, et al. Circulatory support for long-term trearment of heart failute: experience with an intraventricular continuous flow purmp. Circulation 2002; 105:2588-2591

36 Clark RE, Zafirelis Z. Future devices and directions. Prog Cardiovasc Dis 2000; 43:95-100

37 Dowling RD, Etoch SW. Clinically available extracorporeal assist devices. Prog Cardiovase Dis 2000; 43:27-36

38 McCarthy PM, Hoerchet $K$. Clinically available intracorporeal left ventricular assist devices. Prog Cardiovasc Dis 2000; 43:37-46

39 El-Banayosy $A$, Korfer $R$, Arusoglu $L$, et al. Device and patient management in a bridge-to-transplant setting. Ann Thorac Surg 2001; 71:S98-102; discussion S114105

40 Muller ], Wallukat $G$, Weng $Y G$, et al. Weaning from mechanical cardiac support in patients with idiopathic dilated cardiomyopathy. Circulation 1997; 96:542-549

41 Reul HM, Akdis M. Blood pumps for circulatory support. Perfusion 2000; 15:295311

42 Mahmood AK, Courtney JM, Akdis M, et al. Critical review of curpent left ventricular assist devices. Perfusion 2000; 15:399.420

43 Wieselthaler GM, Schima H, Dworschak M, et al. First experiences with outpatient care of parients with implanted axial flow pumps. Artif Organs 2001; $25: 331-335$

44. Thohan V, Stetson S], Nagueh SF, et al. Cellulat and Hemodynamics Responses of Failing Myocardium to Continuous Flow Mechanical Circulatory Support Using the DeBakey-Noon Left Ventricular Assist Device: a Comparative Analysis With Pulsatile-Type Devices. J Heart Lung Transplant 2005; 24:566-575

45. Khanwilkar $P$, Olsen $D$, Bearnson $G$, et al. Using hybrid magnetic bearings to completely suspend the impeller of a ventricular assist device. Artif Organs 1996; $20,597 \times 604$ 
Hetzer $\mathrm{R}$, Weng $\mathrm{Y}$, Potapov $\mathrm{EV}$, et al. First experiences with a novel magnetically suspended axial flow left wentricular assist device. Eur] Cardiothonac Surg 2004; 25:964-970

7 Puers $R$, Vandevoorde $G$. Recent progress on transcutaneous energy transfer for total artificiall heart systems. Artif Organs 2001; 25:400-405

8 Gracin N, Johnson MR, Spokas D, et al. The use of APACHE II scores to select candidates for left ventricular assist dewice placement. Acute Physiology and Chronic Health Evaluation. J Heart Lung Transplant 1998; 17:1017-1023 Roell W, Goedje $\mathrm{O}$, Vetter $\mathrm{HO}$, et al. Improvement of heart-, lung-, and liverperformance during mechanical circulatory support by the Novacor-system. Eur. I Cardiothorac Sutg 1997; 11:1045-1051

Martin J, Siegenthaler MP, Friesewinkel $O$, et al. Implantable left ventricular assist device for treatment of pulmonary hypertension in candidates for orthotopic heart transplantation-a preliminary study. Eur J Cardiothorac Surg 2004; 25:971977

1 Reesink KD, Dekker AL, Van Ommen V, et al. Miniature intracardiac assist device provides more effective cardiac unloading and circulatory support during severe left heart failure than intraaortic balloon pumping. Chest 2004; $126: 896$ 902

2 Smalling RW, Chen P, Buja LM. A 56-year-old man with acute-onset pulmonary edema and shock. Circulation 1994; 89:2921-2927

3 Terracciano $\mathrm{CM}$, Hardy J, Birks EJ, et al. Clinical recovery from end-stage heart failure using left-ventricular assist device and pharmacological therapy correlates with increased sarcoplasmic reticulum calcium content but not with tegression of cellular hypertrophy. Circulation 2004; 109:2263-2265

4 Lee WC, Chavez YE, Baket $\mathbb{T}$, et al. Economic burden of heart failure: a summary of recent literature. Heart Lung 2004; 33:362-371

5 Bartlett RH, Roloff DW, Custer JR, et al. Extracorporeal life support: the University of Michigan experience. Jama 2000;283:904-908

6 Doll N, Kiaii B, Borger M, et al. Five-Year results of 219 consecutive patients treated with extracorporeal membrane oxygenation for refractory postoperative cardiogenic shock. The Annals of Thoracic Surgery 2004; 77:151-157

7 Colson P, Ryckwaert F, Saussine M, et al. Monitoring weaning from BIVAD Thoratec with peak oxygen consumption. Ann Thorac Surg 2004; 77:1808-1810

8 Hon $\mathbb{J K}$, Yacoub MH. Btidge to recovery with the use of left ventricular assist device and clenbuterol. Ann Thorac Surg 2003; 75:S36-41

9 Kim HC, Khanwilkar PS, Bearnson GB, et al. Development of a microcontrollerbased automatic control system for the electrohydraulic total artificial heart. IEEE Trans Biomed Eng 1997; 44:77-89 
Kitamura $\mathbb{I}$, Matsuda $\mathbb{K}_{\text {, }}$ Akashi $H$. Adaptive control technique for artificial hearts. IEEE Trans Biomed Eng 1986; 33:839-844

61 Martin JF, Schneider AM, Quinn ML, et al. Improved safery and efficacy in adaptive control of arterial blood pressure through the use of a supervisor. IEEE Trans Biomed Eng 1992; 39:381-388

62 McInnis BC, Guo Z-W, Lu PC, et al. Adaptive control of left ventricular bypass assist devices. TEEE Transactions on Automatic Control 1985; AC-30;322-329

63 Rucht TL Btown R.H, Jeutter $\mathrm{DC}_{\mathrm{p}}$ et all. Identification algorithm for systemic arterial parameters with application to total artificial heart control. Ann Biomed Eng 1993; 21:221-236

64 Sun Y, Beshara M, Lucariello RJ, et all A comprehensive model for right-left heart interaction under the influence of pericardium and baroreflex. Am J Physiol 1997; 272-H1499-1515

65 Werner J, Bohringer D, Hexamer M. Simulation and prediction of cardiotherapeutical phenomena from a pulsatile model coupled to the Guyton circulatory model. IEEE Trans Biomed Eng 2002; 49:430-439

66 Becker $K$, Rau $G$, Kaesmacher $H$, et al. Fuzzy logic approaches to intelligent alarms. TEEE Engineering in Medicine and Biology 1994:710-716

67 Wu Y, Alaire PE, Tao G, et al. A bridge from short-term to long-term left ventricular assist device-experimental verification of a physiological controller. Artif Organs 2004; 28:927-932

68 Vollkron $M$, Schima H, Huber L, et al. Development of a suction detection system for axial blood pumps. Artif Organs 2004; 28:709-716

69 Schima $\mathrm{H}$, Vollkron $\mathrm{M}$, Boehm H, et al. Weaning of rotary blood pump recipients after myocardial recovery: a computer study of changes in cardiac energetics. I Thorac Cardiovasc Surg 2004; 127:1743-1750 Ohuchi K, Kikugawa D, Takahashi K, et al. Control strategy for rotary blood pumps. Artif Organs 2001; 25:366-370

71 De Lazzati C, Darowski M, Ferrari $G$, et al. Computer simulation of haemodymamic parameters changes with left wentricle assist device and mechanical ventilation. Comput Biol Med 2000; 30:55-69

72 Shavit $A$, Gutfinger $C$. Heat engines and the second law of thermodynamics. Thermodynamics. From concepts to applications. London: Prentice Hall, 1995; 108-125 


\section{Chapter 2}

\section{Feasibility study of a fiber-optic system for invasive blood pressure measurements}

\section{Published as:}

Reesink KD, van det Nagel T, Bovelander J, Jansen JR, van der Veen EJ, Schreuder J]. Feasibility study of a fiber-optic system for inwasive blood pressute measurements. Catheter Cardiowasc Interv 2002; 57:272-276 
30 Chapter 2

\section{ABSTRACT}

\section{Backeground}

A comparative study was conducted to evaluate the feasibility of a fiber optic sensor in invasive blood pressure measurements.

\section{Metbods}

Static and wide-bandwidth stimuli were offered to the fiber optic, Millar, Baxter and Sentron devices to measure static transfer function and transient response. Animal experiments focused on offset-drift, dynamic accuracy and electromagnetic sensitivity.

\section{Results}

Compared to the Millar, Sentron and Baxter devices the fiber optic sensor had a near identical static transfer function. Gain and offset errors were $<3.4 \%$ and $<0.25 \%$, respectively. Hysteresis nonlinearity was $<0.1 \%$. The dynamic accuracy of the fiber optic system matched that of the Millar and Sentron systems. Time delay was $<1 \mathrm{~ms}$. Maximum rate-of-change was $>30,000 \mathrm{mmHg} / \mathrm{s}$ and bandwidth was $0-150 \mathrm{~Hz}$ minimum. Offset-drift was $0.6 \pm 0.03 \mathrm{mmHg}$. Application of diathermy highlighted the fiber optic sensor's excellent electromagnetic disturbance rejection.

\section{Conclusion}

The fiber optic system appears to be a reliable, high-fidelity pressure monitor in demanding clinical environments. 


\section{INTRODUCTION}

Improvements in fiber optic pressure sensor technology have led to the development of a system that employs optical spectrum modulation and fiber optic data transmission!. The fiber optic pressure sensor (FOS) has reached the standards of efficient and economic manufacture recently and might become an alternative product in the field of high-fidelity catheter tip pressure sensors. Pressure sensing catheters are used in cardiology, radiology, cardiac and general surgery, critical care applications, biomedical research and pre-clinical device studies ${ }^{2-4}$.

To evaluate the feasibility of the FOS device, a comparative study was conducted. Two different types of tests were carried out. An in vitro bench test was designed, focusing on the static and dynamic technical properties ${ }^{4}$. In addition, in vivo tests were carried out in animal experiments to simulate clinical conditions. The collected data was used to examine the technical properties in the clinical environment, like static and dynamic accuracy and electromagnetic disturbance sensitivity.

\section{METHODS}

\section{Materials}

The following equipment and test devices were associated with the study. Blood pressure measurement systems (BPMS): 1) RJC ILED-Microshift catheter tip FOS stylet 40EC with model 440 processing unit (RJC Enterprises, Woodinville, WA, USA), 2) Millar Mikro-Tip stylet catheter tip sensor SPC-320 with bridge amplifier (Millar Instr., Houston, TX, USA), 3) Sentron catheter tip sensor with 811-000/A processing unit (CD Leycom, Zoetermeer, The Netherlands), 4) Baxter Uniflow external pressure transducer with bridge amplifier (Baxter Healthcare Ltd.). "T'est signal generator equipment: Bio-Tek Model 601A blood pressure systems calibrator (Bio-Tek Instr., Winooski, VT, USA); $50 \mathrm{MHz}$ function generator; $100 \mathrm{MHz}$ dual-channel digitising oscilloscope; calibration mercury manometer (Hugo Sachs Elektronik, Hugstetten/Breisgau, 
Germany). All pressure signals were digitised at a sampling rate (SR) of 250 samples/s or 1000 samples/s using a Keithley/Metrabyte DAS16G A/Dmodule (Keithley Instr., Cleveland, Ohio, USA).

\section{In vitro}

The three catheter tip pressure sensors were introduced in the calibrator cavity through short, low compliance extension pieces that employ Luerlocks and rubber sealing. The external pressure transducer was not used in conjunction with a fluid filled catheter lumen as usual, but directly connected to the calibrator's pressure dome. The cavity was filled and flushed with an (incompressible) $0.9 \% \mathrm{NaCl}$ saline solution. The function generator drove the calibrator's electrical input. The reference mercury manometer facilitated calibration.

The calibrator's electrical input was driven by a triangle waveform, varying the cavity pressure along a range of -50 to $+180 \mathrm{mmHg}$ (span $=$ $230 \mathrm{mmHg}$ ), to obtain the static transfer function of the BPMS. A triangle drive signal period $T_{d}=1 \mathrm{~s}$ was selected, to provide an appropriate stimulus speed for quasi-static measurement. The digital acquisition resolution was: [range span] $/\left(1 / 2 \cdot T_{d} \cdot S R\right)=230 /(1 / 2 \cdot 1 \cdot 250)=1.84 \mathrm{mmHg}$. Between +180 and $+240 \mathrm{mmHg}$ pressures were measured in steps of $20 \mathrm{mmHg}$ using the mercury manometer. Linear regression fit expressions of the form $k \cdot x+m$ with correlation coefficients $\mathrm{R}^{2}$ were determined using the TRENDLINE option in MS Excel.

The transient response of the BPMS was evaluated by driving the calibrator with a step-up step-down pulse signal (from 40 to 100 back to 40 $\mathrm{mmH} \cdot \mathrm{g}$ ). The pulse repetition period $\left(\mathrm{T}_{2}\right)$ was set to $100 \mathrm{~ms}$ and an electrical slope transition time $\left(\tau_{\text {slopu }}\right)$ of $50 \mathrm{~ns}$ was selected. A sampling rate of 1,000 samples/s was selected to obtain a resolution of $1 \mathrm{~ms}$. The following parameters and features were processed from the recorded waveforms: time delay, rise and fall times, percentage overshoot, oscillation frequency and hysteresis. 


\section{In vivo}

The in vivo protocol was conducted in two goats (mass 55 and $72 \mathrm{~kg}$ ). The experiments were performed according to the "Guide for the Care and Use of Laboratory Animals" published by the US National Institutes of Health [NIH publication 86-23, Revised 1985] and approved by the Animal Ethical Committee of the University of Maastricht. The animals were anaesthetised with Nesdonal, $\mathrm{O}_{2}: \mathrm{N}_{2} \mathrm{O}=1: 2$, halothane: $1.0-2.5 \%$ and were ventilated at a respiratory rate of $15 \mathrm{~min}^{-1}$ with tidal volumes adjusted to keep expiratory $\mathrm{CO}_{2}$ between 4 and $5 \%$. The animals received buprenorfine preand peroperative intramuscular, a bolus of $10,000 \mathrm{IU}$ of heparin iv and 5,000 IU of heparin per $500 \mathrm{ml}$ Ringer's lactate iv for anti-coagulation.

The Sentron catheter was introduced via a carotid artery sheath and advanced into the aortic arch at a $20-40 \mathrm{~mm}$ distance from the proximal part of the descending aorta. A diagnostic catheter was introduced via a femoral artery sheath and then placed in the descending aorta, with the tip spaced 30-50 $\mathrm{mm}$ from the aortic arch. The Millar and FOS stylets were inserted through the diagnostic catheter's internal lumen and the sensors were positioned close to the tip. Catheter manipulation and alignment were assisted by X-ray imaging. The data acquisition sampling rate was set to 250 $\mathrm{Hz}$.

Dynamic properties: 'To study the dynamic signal response of the BPMS in vivo, aortic blood pressure recordings were made. Particular attention was paid to waveform representation quality (time-delay, rate-ofchange). Offset-drift: During the two animal experiments pre and post experiment calibrations focused on offset stability over a time interval of more than two hours. EM disturbance sensitivity: To investigate the behaviour of the pressure sensors and the connected processing equipment in an electromagnetically hostile environment, a basic surgery procedure was carried out. Diathermy incisions and coagulation were applied to thorax tissue, while recording the signals produced by the sensors. 


\section{RESULTS}

The results are given as mean $\pm \mathrm{SD}$ (group; four devices).

\section{In vitro}

Static transfer function data are presented in Table 2.1. The number of data points used in the linear regression fit expression was 125. Fit coefficients were $k=0.9848 \pm 0.015$ and $m=-0.258 \pm 1.58 \mathrm{mmHg}$. All correlation coefficients $\mathrm{R}^{2}$, but one, were greater than 0.9995 . The smaller correlation found with the FOS data $\left(R^{2}=0.9989\right)$ was related to the non-linear clipping behaviour at the negative range extreme. Linear regression analysis of the FOS data on a restricted range from -20 to $+180 \mathrm{mmHg}$ yielded $k=$ $0.9698,|\Delta k|=3.1 \%$ and $m=0.087 \mathrm{mmHg},|\Delta m|=0.038 \%$ and $\mathrm{R}^{2}=$ 0.9997 . So, $15 \%$ of the gain error is related to non-linear clipping in the negative range $(<-20 \mathrm{mmHg})$. Figure 2.1 depicts the FOS system's transfer

Table 2.1 Static transfer function data

\begin{tabular}{|c|c|c|c|c|c|}
\hline Device & $k+m(\mathrm{~mm} / \mathrm{Hg})$ & $R^{2}$ & $\begin{array}{c}|\Delta k| \\
(\%)\end{array}$ & $|\Delta m|(\%)$ & $\begin{array}{c}\Delta a_{n-p} \\
(m m H g)\end{array}$ \\
\hline Millat & $0.9812 \cdot x+1.04$ & 0.9999 & 1.9 & 0.452 & $<$ resolution \\
\hline Sentron & $1.001 \cdot x-2.54$ & 0.9996 & 0.10 & 1.11 & $<$ resolution \\
\hline FOS & $0.9657 x+0.469$ & 0.9989 & 3.4 & 0.204 & $<$ resolution \\
\hline Baxter & $0.9917 \cdot x+0$ & 1.0000 & 0.83 & 0 & $<$ resolution \\
\hline $\begin{array}{l}\text { k:x } x= \\
\text { mmHg), } k \\
\text { (deviation } \\
\Delta a_{\mathrm{w}-\mathrm{p}}=\text { hys }\end{array}$ & $\begin{array}{l}\text { ain, regression fi } \\
\text { 1), }|\Delta m|=\mathrm{abs} \\
\text { sis. }\end{array}$ & $\begin{array}{l}\text { pressio } \\
\text { correl } \\
\text { offset }\end{array}$ & $\begin{array}{l}=\text { inp } \\
\text { oeffic } \\
\text { (tatio }\end{array}$ & $\begin{array}{l}\text { essure; ra } \\
|\Delta k|=\text { : } \\
\text { eviation } f\end{array}$ & $\begin{array}{l}-50 \text { to }+180 \\
\text { hute gain error } \\
0 \text { to full scale, }\end{array}$ \\
\hline
\end{tabular}


function and linear regression approximation build from the data of one run $(-50$ to $+180 \mathrm{mmHg} ; 170$ data points). The additional mensurements in the range from +180 to $+240 \mathrm{mmHg}$ ( $\mathrm{n}=2,4$ data points), measured under genuine static conditions, implicated a straight forward extrapolation. The FOS system's static transfer was approximately linear from -40 to $+240 \mathrm{mmHg}$. Possible hysteresis could not be quantified in the static transfer function data. Only small hysteresis nonlinearity was found in the transient response data: $\Delta a_{a-p}=0.017 \pm 0.031 \%(n=2)$.

Transient response data are presented in Table 2.2. Time delay was determined as the number of samples recorded from the stimulus onset until some change in the measured response occurred: $t_{d}=1.5 \pm 1 \mathrm{~ms}$. The Sentron system's additional delay of $2 \mathrm{~ms}$ is a property of its electrical isolation circuitry. Rise and fall times were determined counting the number of samples from response change onset until the steady-state value was first

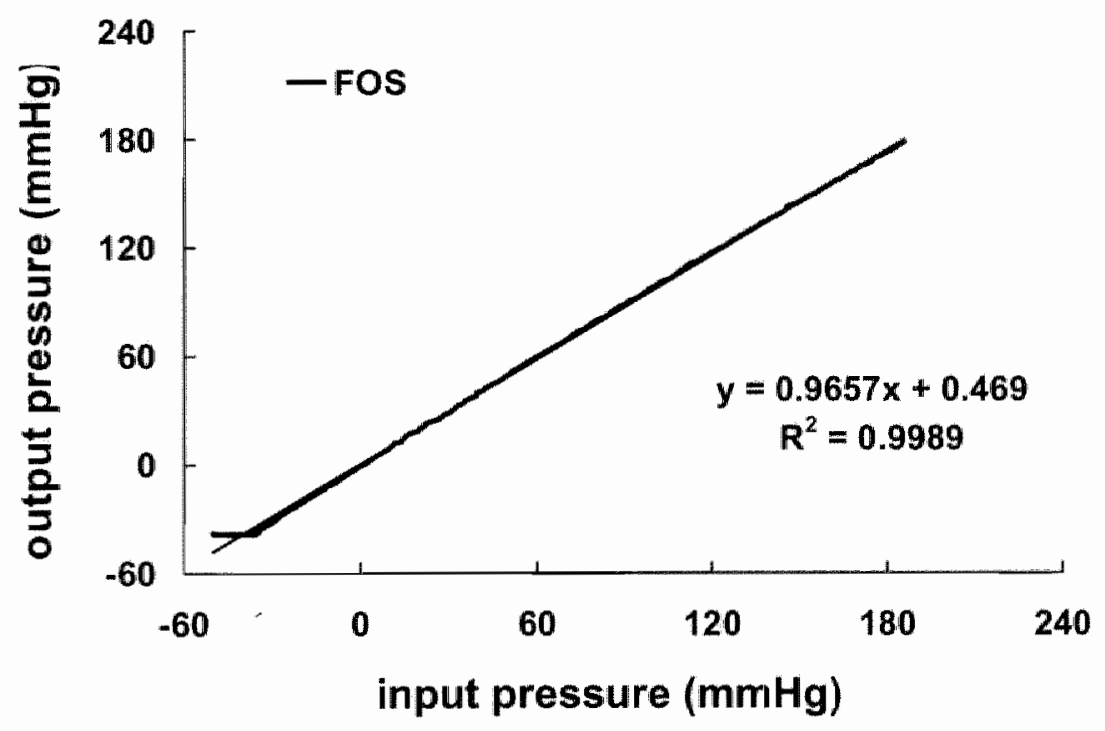

Figure 2.1 Static transfer function and linear regression fit. 
361 Chapter 2

Table 2.2 Transient response data

\begin{tabular}{|c|c|c|c|c|c|}
\hline Device & $\mathrm{d}(\mathrm{ms})$ & $\tau_{i}=\tau_{r}(\mathrm{~ms})$ & P.O. $(\%)$ & $\Delta \mathrm{at}_{\mathrm{a}-\mathrm{p}}(\mathrm{m} \times \mathrm{m} \mathrm{Hg})$ & $\Delta \mathrm{x}_{\mathrm{a}-\mathrm{p}}(\%)$ \\
\hline Millat & 1 & 2 & 59 & 0.13 & 0.057 \\
\hline Sentron & 3 & 3 & 52 & 0.0045 & 0.0020 \\
\hline $\mathrm{FOS}$ & 1 & 2 & 50 & 0.036 & 0.017 \\
\hline Baxter & 1 & 2 & 59 & 0.052 & 0.023 \\
\hline
\end{tabular}

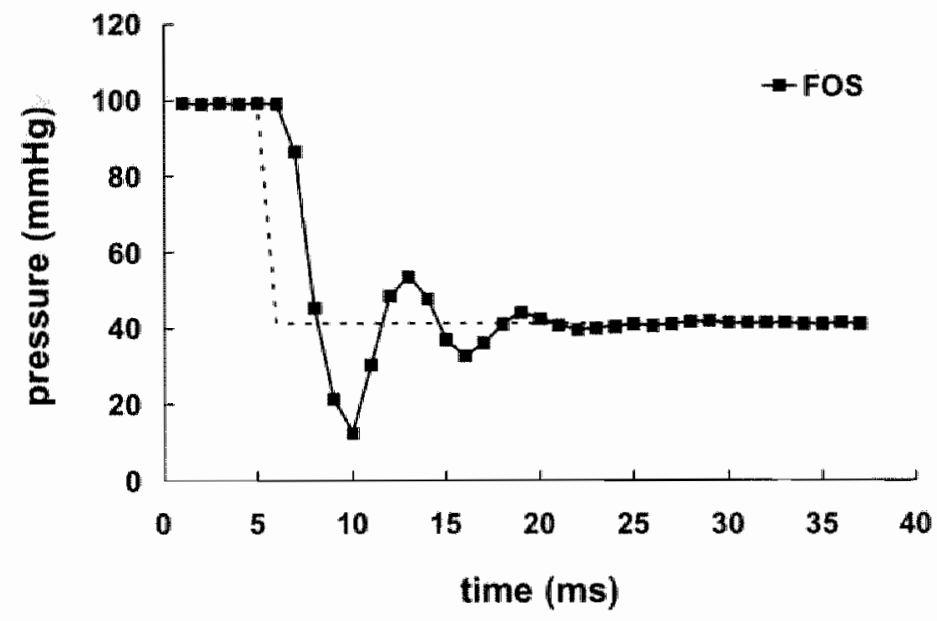

Figure 2.2 Transient response with stimulus signal for reference.

reached: $\tau_{\mathrm{r}}=\tau_{\mathrm{i}}=2.25 \pm 0.5 \mathrm{~ms}$. The maximum rate-of-change can be expressed in $\Delta \mathrm{P} / \Delta \mathrm{t}=$ [step height] $/ \tau: 27.5 \pm 5.0 \cdot 10^{3} \mathrm{mmHg} / \mathrm{s}$. The overshoot P.O. $=55 \pm 4.7 \%$ seen in the transient response diagram in Figure 2.2 was caused by speed limitations of the calibrator system that led to under-damped oscillation. The oscillation frequency was $\mathrm{f}_{\mathrm{osc}} \approx 167 \mathrm{~Hz}$, 
sustaining the $0-150 \mathrm{~Hz}$ usable bandwidth clained in the calibrator's user manual. The FOS system has a bandwidth extension to at least $150 \mathrm{~Hz}$.

\section{In vivo}

Dynamic properties: Figure 2.3 shows an aortic pressure recording of the Millar, FOS and Sentron systems. Only one beat is depicted to focus on the slopes, rates-of-change and the dicrotic notch of the waveform as impottant physiological markers. The Millar and FOS traces differ slightly from the Sentron signal, in the upper part of the systolic upstroke. Correlation of the Millar with the FOS data indicated a near identical dynamic performance in clinical practice: $\mathrm{R}^{2}=0.9978$. Offset-drift: The changes in ambient pressure read-out of the FOS system were calculated to measure offset-drift: $\Delta m=$ $0.6 \pm 0.03 \mathrm{mmHg}(\mathbf{n}=2)$ over two hours. The body temperature was approximately $37^{\circ} \mathrm{C}$ and fluctuated within $1{ }^{\circ} \mathrm{C}$ over time.

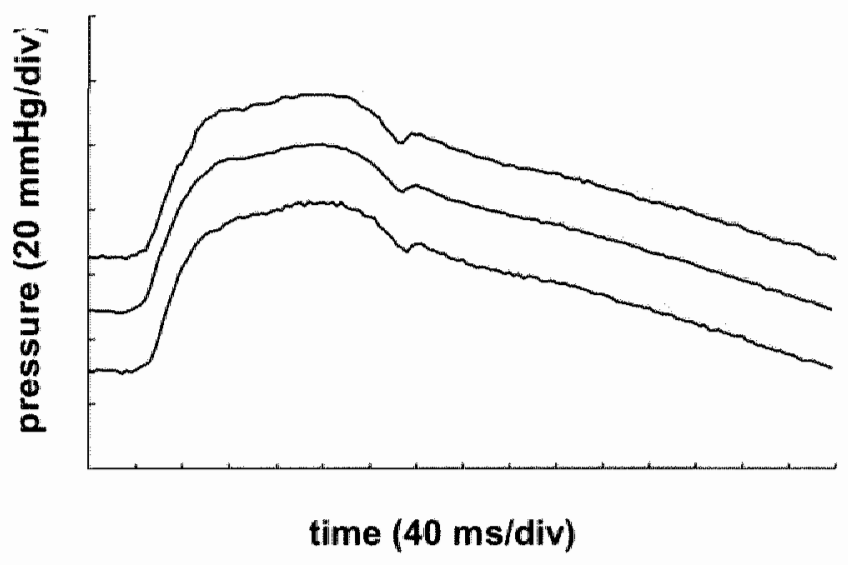

Figure 2.3 Recording of aortic pressure. Tracings are shifted vertically to avoid overlap. Top: Millar, middle: FOS, bottom: Sentron. 
EM disturbance sensitivity: During the application of diathermy the pressure signal of the Millar sensor drove the processing equipment out of range, so that no valid pressure values could be determined (Figure 2.4). At the bottom of the diagram a scaled ECG signal is given for reference. The FOS system delivered unaltered performance: the optical blood pressure signal remained intact. Routinely, the Sentron sensor lead was temporarily disconnected to avoid damage to the processing interface (manufacturer's advice).

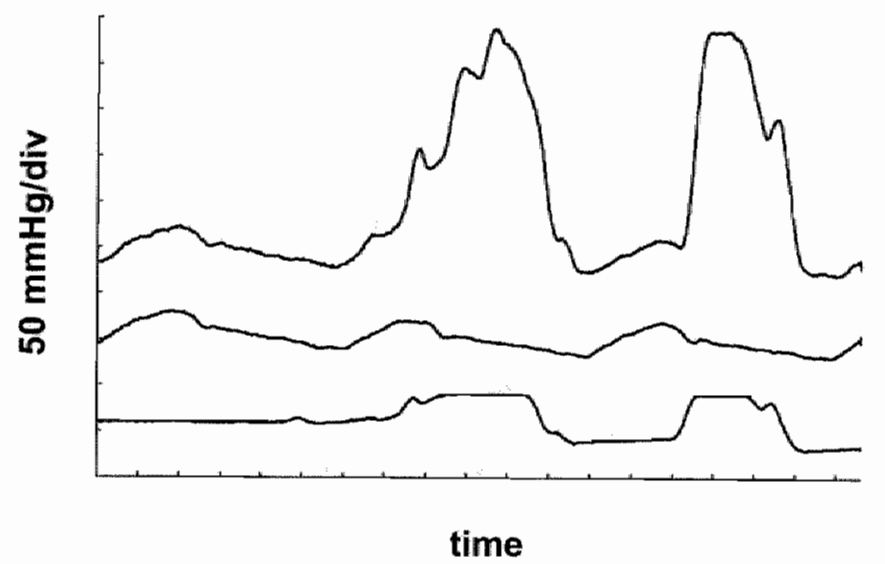

Figure 2.4 Effect of diathermy on measured pressure signals. Tracings are shifted vertically to avoid overlap. BCG trace is shown for reference. Top: Millar, middle: FOS, bottom: ECG. 


\section{DISCUSSION}

\section{In vitro}

A major limitation of this bench test was bandwidth, which was directly related to response speed. The calibrator's usable bandwidth is specified from $0-150 \mathrm{~Hz}$, which is also supported by the data. Therefore the actual upper bandwidth extremes could not be determined using this calibrator. Although this set-up did not address the full bandwidth of the BPMS tested, it certainly delivered reliable and accurate data to investigate the feasibility of the FOS in high fidelity blood pressure monitoring. Blood pressure measurements require a bandwidth of $0-80 \mathrm{~Hz}$ in standard catheterisation procedures and experimental large animal and patient studies, considering a maximum heart rate of $240 \mathrm{bpm}(4 \mathrm{~Hz})$ plus an additional 20 harmonics required if data processing involves derivation of $\mathrm{dP} / \mathrm{dt}^{3,5,6}$. Literature reports a flat bandwidth up to $1000 \mathrm{~Hz}$ for the FOS '.

The time delay $\left(t_{d}=1.5 \pm 1 \mathrm{~ms}\right)$ and the rise and fall times $(\tau=2.25$ $\pm 0.5 \mathrm{~ms})$ are in the same order of magnitude as the resolution, which is 1 ms. These parameter values may be smaller than indicated and therefore they are considered maximum values.

Dynamic accuracy is of great importance in intra-aortic balloon pump assistance ${ }^{7,8}$. Only wide-bandwidth pressure signal acquisition and processing can enable correct identification of the dicrotic notch (closing of the aortic valve) in an aortic pressure signal. The IABP's control software uses this identification to tune assist parameters. A high quality left ventricular pressure signal is also essential for advanced hemodynamic monitoring like P-V loop measurement. P-V Loop analysis offers an estimation of left ventricular contractility and mitral and aortic valve functions ${ }^{9,11}$.

The static transfer properties were evaluated in the range of -50 to $+180 \mathrm{mmHg}$ with a fairly high resolution of $1.84 \mathrm{mmHg}$. When ambient pressure is used as the $0 \mathrm{mmHg}$ reference, small negative pressures with an order of magnitude of $-10 \mathrm{mmHg}$ can be seen occasionally in left 
401 Chapter 2

ventricular pressure measurements just after the ejection phase of the cardiac cycle. A range extension below $0 \mathrm{mmHg}$ is desirable and will also provide a margin for offset deviations, which can be corrected during data processing. The non-linear behaviour of the FOS's static transfer below -20 $\mathrm{mmHg}$ was incorporated in the linear regression analysis. Exclusion of the -50 to $-20 \mathrm{mmHg}$ sub range in the analysis decreases both gain and offset errors: $|\Delta k|$ from 3.5 to $3.1 \%$ and $|\Delta m|$ from 0.21 to $0.04 \%$. The upper limit ( $+180 \mathrm{mmHg}$ ) of the method's amplitude range is considered to be high enough to accommodate the maximum blood pressures found in humans, also in case of (uncomplicated) hypertension ${ }^{6}$. Cases of hypertension complicated by aortic valve stenosis put a greater demand on upper range extension. Therefore we extended this range to $+240 \mathrm{mmHg}$ to investigate possible transfer anomalies at the upper range extreme.

No hysteresis was found in the static transfer data, so any hysteresis present is assumed to be only a fraction of the resolution $(1.84 \mathrm{mmHg})$. The absence of significant hysteresis was sustained by the transient response data, that indicated only very small hysteresis $<0.15 \mathrm{mmHg}(<0.065 \%)$, which was more likely the result of residual noise. This value is well below the calibrator's maximum full scale nonlinearity error specification of 2.5 $\mathrm{mmHg}(0.83 \%$; full scale $=-50$ to $+250 \mathrm{mmHg})$.

\section{In vivo}

The baseline recording of aortic pressure shows great similarity of the Sentron, Millar and FOS waveforms. The additional time delay of the Sentron system ( $2 \mathrm{~ms}$ ) found in vivo is in accordance with the in vitro data. This static time delay being the only major difference with the rest of the devices, the Sentron system is still considered a valid reference for dynamic accuracy. It has earned a solid reputation after numerous previous animal studies ${ }^{9-11}$.

The offset-drift of the FOS system was measured over a restricted period of time (two hours); longer measurement sessions should reveal more reliable drift coefficients. The drift was calculated from two data 
points (pre and post calibration values) with $n=2$. This provides only little information on drift properties during the interval. The effects of parameters like temperature and blood composition should be studied in more detail to obtain all drift coefficients. However, these results could indicate, that there are no impractical drift anomalies to be expected.

The high energy/high frequency electromagnetic fields associated with modern surgical tools are a source of EM disturbance. Most current electromechanical sensors are particularly susceptible to EM interference. As an alternative to electromechanical principles the fiber optic system uses optical sensing and transmission techniques: the sensitive electrical processing circuitry is effectively isolated from the disturbance source by fiber optic data transmission. This also implicates inherent electrically safe operation (cardiac floating), because the optical isolation is bi-directional. The risk of a hazardous fault current that may induce ventricular fibrillation (cardiac arrest) is eliminated. It is evident also, that loss of valid data duting the application of diathermy rules out proper hemodynamic monitoring and control of a cardiac assist device like the IABP.

\section{Acknowledgement}

We thank J. Habets for his assistance and skills.

\section{References}

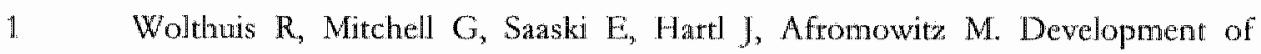
Medical Pressure and Temperature Serrsors Employing Optical Spectrum Modulation. IEEE Transactions on Biomedical Engineering. 1991; 38:974-981.

2 Brown JM, Carr JJ. 1981. Introduction to biomedical equipment rechnology. New York: Wiley. $430 \mathrm{p}$

3 Nichols WW, Pepine CJ, Cont CR. Catheter tip manometer system: "pressure and velocity measurement. Adv Cardiovasc Phys. 1983; 5:144-158

4 Milnot WR. 1982. Hemodynamics. Battimore: Williams \& Wilkins. 272-292

5 Gersh B], Hahn CEW, Roberts. CP. Physical criteria for measurement of left ventricular pressure and its derivative. Cardiovasc Res 1971; 5:32-40

6 Geddes LA. 1970. The direct and indirect measurement of blood pressure. Chicago: Year Book Medical Publishers. 128-130 
Plummer PM. Biomedical engineering fundamentals of the intra-aorric balloon pump. Bioned Instrum Technol 1989; $23: 452-459$

8 Helman DN, Vahakes GJ. 2000. Intracoporeal support: the intra-aortic balloon pump. In: Goldstein DJ and $\mathrm{Oz} \mathrm{M}$, editors. Cardiac assist dewices. New York: Futura Publishing Company. 291-306

9 Schreuder JJ, van det Veen FH, van der Velde ET, Delahaye F, Alfieri $O$, Jegaden $O$, Lotusso R, Jansen JRC, van Ommen V, Finet $G$, Wellens HJJ. Beat-to-beat analysis of left ventricular pressure-volume relation and stroke volume by conductance catheter and aortic model flow in cardiomyoplasty patients. Circulation $1995 ; 91: 2010-2017$

10 Schreuder JJ, van der Veen FH, van der Velde ET, Delahaye F, Alfieri O, Jegaden O, Lorusso R, Jansen JRC, Hoeksel SAAP, Finet G, Voltertani M, Kaulbach $H$ $G$, Baan J, Wellens HJJ. Left ventricular pressure-xolume telationships before and after cardiomyoplasty in patients with heart failure. Circulation 1997; 96:29782986

11 Dekker AL, Geskes GG, Cramers AA, Dassen WR, Maessen JG, Prenger KB, wan der Veen FH. Right ventricular support for off-pump coronary atrery bypass grafting studjed with bi-ventricular pressure-volume loops in sheep. European Journal of Cardio-thoracic Surgery 2001; 19:179-184 


\section{Chapter 3}

\section{Synchronously counterpulsating extracorporeal life support enhances myocardial working conditions regardless of systemic perfusion pressure}

\section{Accepted for publication as:}

Reesink KD, Sauren LDC, Dekker ALAJ, Severdija E, wan der Nagel 'T, Geskes GG, wan der Veen FH, Maessen JG. Synchronously counterpulsating extracorporeal life support enhances myocardial working conditions regardless of systemic perfusion pressure. Eur J Cardiothotac Surg. In press. 
$44 \mid$ Chapter 3

\section{ABSTRACT}

\section{Backeground}

A new pulsatile extracorporeal life support (pECLS) system has entered the market. We wanted to investigate what potential advantages pECLS may have over current non-pulsatile systems (NPS). Our research was focused on the pump's functionial interaction with the left ventricle and the coronary circulation.

\section{Methods}

Extensive hemodynamic measurements were performed during asynchronous and synchronous pECLS in ten calves. The two extremes regarding LV afterload, namely systolic arrival (SA) and diastolic arrival (DA) of the pump pulse were studied.

\section{Results}

SA was associated with increased oxygen consumption $(+57 \%)$ and decreased diastolic coronary perfusion $(-43 \%)$. DA increased left ventricular output (4.5 \pm 2.4 versus $\mathrm{SA}: 3.5 \pm 2.2 \mathrm{l} / \mathrm{min}), \mathrm{LV}$ ejection fraction $(+10 \%)$, and ventricular efficiency $(+17 \%)$. Mean aortic pressure and mean coronary flow were only marginally affected by pulse incidence. Systolic impairment was more pronounced with higher bypass flows. These results indicate that myocardial working conditions can be optimized by phasing pECIS ejection into cardiac diastole.

\section{Conclusion}

We conclude that during pECIS myocardial working conditions can be improved by avoidance of systolic impairment. Synchronously counterpulsating pECLS could be a more economic and versatile alternative to NPS or NPS combined with intra-aortic balloon pumping. The potential benefits of synchronously counterpulsating pECLS over the current alternatives remain to be investigated. 


\section{INTRODUCTION}

From 1965 on, adult extracorporeal membrane oxygenation (ECMO, or more generic in literature: ECLS) has provided a means to support patients suffering from postcardiotomy cardiopulmonary failure with typical durations that range from a few hours to a couple of weeks ${ }^{12}$. More recently, the use of ECLS to assist high risk minimally invasive surgical procedures has gained more interest ${ }^{3.5}$.

In today's clinical practice mostly non-pulsatile ECLS circuits are employed. As set forth by Wright and others, the choice for this technology is bound to a historical context ${ }^{6,7}$. In the early years pulsatile circuits were bulky and expensive systems which required elaborate setup and tuning. The advent of roller pumps and later centrifugal pumps paved the way for smaller, cheaper, and easier to setup non-pulsatile systems. However, little scientific evidence was available to back up the trend towards continuous flow perfusion because research efforts lagged behind the technological progress. Today, the debate remains controversial as solid evidence is still void $^{7 \cdot 10}$.

The application of (continuous flow) centrifugal pumps in ECIS systems has gained widespread use clinically ${ }^{6,11}$. Whereas continuous flow technology has valuable features like good reliability and lower cost, ECLS therapy is frequently combined with intra-aortic balloon pumping, which suggests that the specific cardiac/coronary demands cannot always be met by the extracorporeal circuit alone 13,5,11. $^{\text {. }}$

We sought to investigate the mechanics of pulsatile extracorporeal life support (pECLS) and specifically the hemodynamic interaction with the left ventricle and coronary circulation. Our hypothesis is that left ventricular function is impaired when extracorporeal pulse flow occurs during cardiac systole and that this will adversely affect myocardial working conditions. 
46. Chapter 3

\section{METHODS}

\section{Animal preparation}

All animals received humane care in compliance with the "Guide for the Care and U'se of Laboratory Animals" (NIH publication 86-23, 1985 revision; National Institutes of Health, USA). Ten calves weighing $75 \pm 26$ $\mathrm{kg}$ (mean $\pm \mathrm{SD}$ ) were premedicated with atropine $(0.05 \mathrm{mg} / \mathrm{kg}$ s.c.). Anesthesia was induced with sodium thiopental (bolus $20 \mathrm{mg} / \mathrm{kg}$ i.v.) and maintained with a $1: 2$ mixture of $\mathrm{O}_{2}: \mathrm{N}_{2} \mathrm{O}$ and halothane $(1.5 \%$ ). After administration of analgesic buprenorfine (bolus $0.01 \mathrm{mg} / \mathrm{kg} \mathrm{i.v.)}$ ) and muscle relaxant suxamethonium (bolus $0.1 \mathrm{mg} / \mathrm{kg}$ i.v.), a left thoracotomy was performed while maintaining a peak end-expiratory pressure of $5 \mathrm{~cm} \mathrm{H}_{2} \mathrm{O}$. Coagulation was controlled through administration of heparin (bolus 200 $\mathrm{IU} / \mathrm{kg}$ i.v.) and monitored by activated clotting time (ACT) measurements. ACT was kept above 480 seconds during the experiment. Monitoring included ECG, blood pressure, oxygen saturation and capnography. The animal was sacrificed through a pentobarbital overdose (bolus $80 \mathrm{mg} / \mathrm{kg}$ i.v.). The study was approved by the animal ethical committee of the University of Maastricht.

\section{Circulatory support}

The T-PLS system is a recently developed ECLS system (NewheartBio Corporation, Seoul, Korea) which features two tubular shaped pumping chambers in push-pull configuration ${ }^{12,13}$. The pumps fill passively and are emptied by an undulating pendulum which pushes the sacs in alternating mode. The maximum volume of the chambers is $70 \mathrm{ml}$. The pump rate is specified as selectable from 20 to $120 \mathrm{bpm}$ in steps of $1 \mathrm{bpm}$, but cannot be synchronized by external inputs. The system features a suction alarm and a feedback circuit that reduces pump rate to avoid severe suction.

The ECLS system was configured as a femoro-femoral veno-arterial ECMO circuit which was primed through the use of a venous reservoir. 


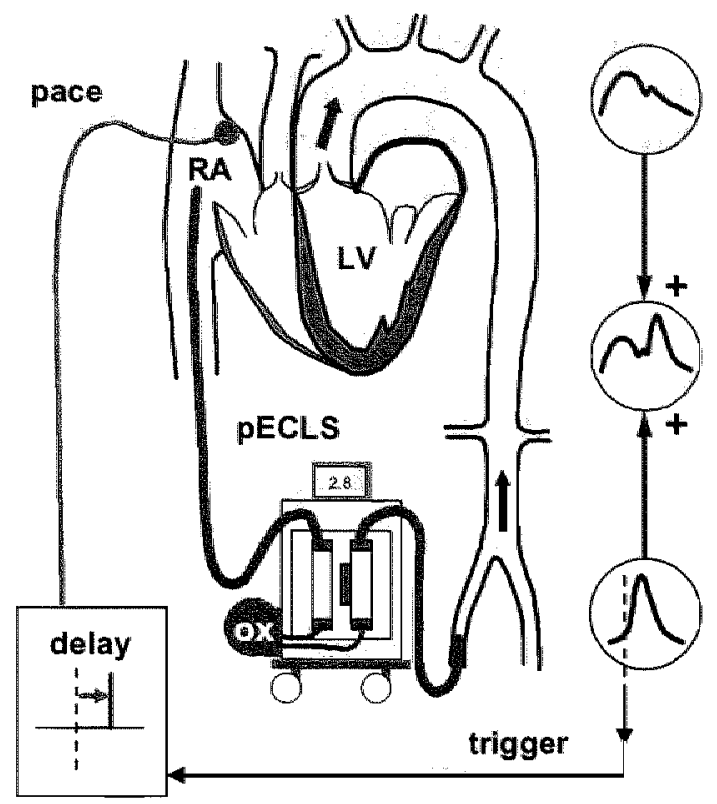

Figure 3.1 Basic experimental setup. RA, right atrium; $L V$, left ventricle; pECLS, pulsatile extracorporeal, dife support system. The diagram gives an interpretation of how a pulse coming from the extracorporeal system is superimposed on the cardiac pulse. For synchronization purposes a pacing loop was configured as follows: A trigger was derived from the pump outflow signal, which was then converted to an atriall pace stimulus after the application of a selectable delay.

During support the reservoir was bypassed ${ }^{14}$. Femoral arterial access was via a 21 Fr cannula (Jostra, Hirrlingen, Germany). Either a femoral vein, jugular vein, or both were cannulated for venous drainage $(24-27 \mathrm{Fr}$ or $32 / 40 \mathrm{Fr}$ double stage venous cannulae). An external pressure sensor (Baxter International. Inc., Deerfield, IL) and an ultrasonic flow probe (Transonic Systems, Ithaca, NY) were placed on the arterial cannula to measure pump output pressure and flow. Pump output was adjusted to 40$60 \%$ of total output. Figure 3.1 sketches the basic experimental arrangement and presents the idea of interacting pulses from the heart and the extracorporeal circuit. 
48. Chapter 3

\section{Instrumentation}

A Swan-Ganz thermodilution catheter (Arrow International, Reading, PA) was advanced into the pulmonary artery via a femoral vein. An external pressure transducer was placed on the central venous lumen (Baxter International Inc., Deerfield, IL). A conductance catheter, incorporating a pressure sensor (CD Leycom, Zoetermeer, the Netherlands), was positioned in the left ventricle via left femoral arterial access. The conductance catheter was connected to a Sigma 5DF conductance processor (CD Leycom, Zoetermeer, the Netherlands) which was used in dual-field mode ${ }^{15}$. Solidstate pressure catheters (CD Leycom, Zoetermeer, the Netherlands) were placed in the ascending aorta via the left carotid artery and in the left atrium through a purse-string suture. Ultrasonic flow probes (Transonic Systems, Ithaca, NY) were placed on the ascending aorta, and on a side branch of the left anterior descending coronary artery. All hemodynamic data were acquired and stored using a 16-channel acquisition system (Conduct-PC, CD Leycom, Zoetermeer, the Netherlands).

\section{Conductance calibration}

Parallel conductance was determined by injecting $7.5 \mathrm{ml}$ of $6.5 \%$ hypertonic saline into the pulmonary artery ${ }^{16}$. A $5 \mathrm{ml}$ blood sample was collected in a sampling cuvette for blood resistivity measurement (CD Leycom, Zoetermeer, the Netherlands) ${ }^{17}$. The gain calibration factor was determined by comparing conductance derived left ventricular output with the aortic flow (LVO) at baseline. The conductance data was analysed with the Circlab 2000 software package (Paul Steendijk, Leiden University Medical Center, Leiden, the Netherlands).

\section{Measurements and analysis}

\section{Asynchronows protocol}

Animal heart rate was greater than $90 \mathrm{bpm}$ and the pump rate was set between 60 and 80 bpm. Hereby asynchronous support was established. 
After stabilization of bypass flow and cardiac output; hemodynanic recordings of 30 seconds duration were made. To differentiate between the effects of systolic (SA) and diastolic arrival (DA) of the pECLS pulse at the aortic valve, the associated beats were selected and averaged (at least $>5$ beats of each type).

\section{Synchronous protocol}

Because the T-PLS system supplied for investigation did not provide any means to synchronize its output to the heart beat, the reverse option was employed: A custom cardiac pacing loop was designed around the setup shown in Figure 3.1, and implemented in LabView (National Instruments, Austin, TX). A trigger pulse was derived from the upward slope of the pump outflow signal and subjected to a selectable delay. The delayed signal was then fed to the trigger input of a cardiac pacing unit. Atrial pacing was employed to ensure homogenous cardiac activation. By this arrangement heart rate was synchronized to pumping frequency.

The simultaneous ejection of the heart and the pump was defined as the zero delay setting. Consecutively, the pace pulse was delivered in delayed fashion covering the entire cardiac cycle in steps of $50 \mathrm{~ms}$. With every run hemodynamics were allowed to stabilize before recordings were made.

The following hemodynamic indices were calculated. An estimate of external left ventricular work (EW) was calculated from: (VIv $\left.V l_{\mathrm{ES}}\right) \cdot\left(\mathrm{Pl} \mathrm{V}_{\mathrm{PEAK}}-\mathrm{PlV}_{\mathrm{ED}}\right) \cdot \mathrm{LV}$ potential energy (PE) was estimated by: $1 / 2 \cdot \mathrm{Vlv}_{\mathrm{ES}} \cdot\left(\mathrm{PlV}_{\mathrm{PEAK}}-\mathrm{Pl}_{\mathrm{FD}_{\mathrm{BD}}}\right)$. The total pressure-volume area was calculated from: PVA $=\mathrm{EW}+\mathrm{PE}^{18}$. The ratio of EW over PVA was calculated as a measure of ventricular efficiency ${ }^{19}$. From the synchronous data the percentage of coronary flow during diastolic (DCF, in \%) was calculated. Statistical comparison was performed by application of the non-parametric Wilcoxon signed-ranks test $(n=10)$. Significance was assumed if $p$-values were lower than 0.05 . 
50 . Chapter 3

\section{RESULTS}

Asynchronous measurements were obtained successfully in all animals. During support the T-PLS system pumped $1.9 \pm 0.3 \mathrm{l} / \mathrm{min}$ (mean \pm SD) while mean total cardiac output was $5.6 \pm 2.4 \mathrm{l} / \mathrm{min}$. Among the individual animals total cardiac output varied considerably and thus a substantial range in bypass ratios was covered (pump flow/total cardiac output; range 18-82\%, mean $34 \%$ ). The intended limited range of $40-60 \%$ could not be achieved. During pump installment and operation it was evident that the period available for venous filling (half the pump cycle length) and the bore of the venous cannula were the primary factors limiting venous return and pump output. At high pump rate settings (>90 bpm) the T-PLS system effectively reduced its rate to prevent suction. The maximally attainable pump rate in these experiments was therefore only $80 \mathrm{bpm}$. In the synchronous protocol these limitations necessitated the use of a $\beta$-blocker agent (Inderal, Propanolol, AstraZeneca, Zoetermeer, the Netherlands) to enable pacing at a rate of $80 \mathrm{bpm}$. In 5 animals the procedure proved successful, and stable synchronization of the heart beat to the pECLS system was obtained. During synchronous support the pECLS delivered a flow of $1.9 \pm 0.4 \mathrm{l} / \mathrm{min}$ while the left ventricular output amounted to approximately $1.8 \pm 4.0 \mathrm{l} / \mathrm{min}$. The details and data of both protocols are presented separately below.

\section{Pump system on-off}

In Table 3.1 the hemodynamic effects of unsynchronized support have been summarized. With the pECLS system switched on, central venous pressure decreased and aortic pressure and coronary flow increased significantly: Pcv $-20 \%$, Pasc $+30 \%$, and Qcor $+30 \%(\mathrm{p}<.05) . \mathrm{Pl}_{\mathrm{PEAK}}$ increased from $79 \pm 24$ to $97 \pm 27 \mathrm{~mm} \mathrm{Hg}(\mathrm{p}<.05)$. Peak aortic pressure was elevated beyond peak left ventricular pressure (Pasc PEAK $103 \mathrm{~mm} \mathrm{Hg}>$ PJveAK $97 \mathrm{~mm} \mathrm{Hg}$. 
Table 3.1 Hemodynamic effect of asynchronous support

\begin{tabular}{cccc}
\hline & units & pump off & pump on \\
\hline Qpump & $1 / \mathrm{min}$ & - & $1.9(0.3)$ \\
LVO & $1 / \mathrm{min}$ & $4.4(2.9)$ & $3.7(2.4)$ \\
CO & $1 / \mathrm{min}$ & $4.4(2.9)$ & $5.6(2.4)$ \\
Qcor & $\mathrm{ml} / \mathrm{min}$ & $61(36)$ & $79(48)^{*}$ \\
PlvpEAK & $\mathrm{mm} \mathrm{Hg}$ & $79(24)$ & $97(27)^{*}$ \\
PascPEAK & $\mathrm{mm} \mathrm{Hg}$ & $78(25)$ & $103(27)^{*}$ \\
Pasc & $\mathrm{mm} \mathrm{Hg}$ & $62(17)$ & $81(23)^{*}$ \\
Pcr & $\mathrm{mm} \mathrm{Hg}$ & $11.6(6.8)$ & $9.3(4.3)^{*}$ \\
\hline
\end{tabular}

Values are mean (SD), $\mathrm{n}=9{ }^{*} \mathrm{p}<.05$ versus pump off. Qpump, bypass pump flow; $\mathrm{LVO}$, left ventricular output; $\mathrm{CO}$, total cardiac output; Qcor, coronary artery flow; Plvpeak, peak left ventricular pressure; Pascpenk, peak ascending aortic pressure; $\mathrm{Pasc}$, ascending aortic pressure; $\mathrm{Pcr}$, central venous pressure.

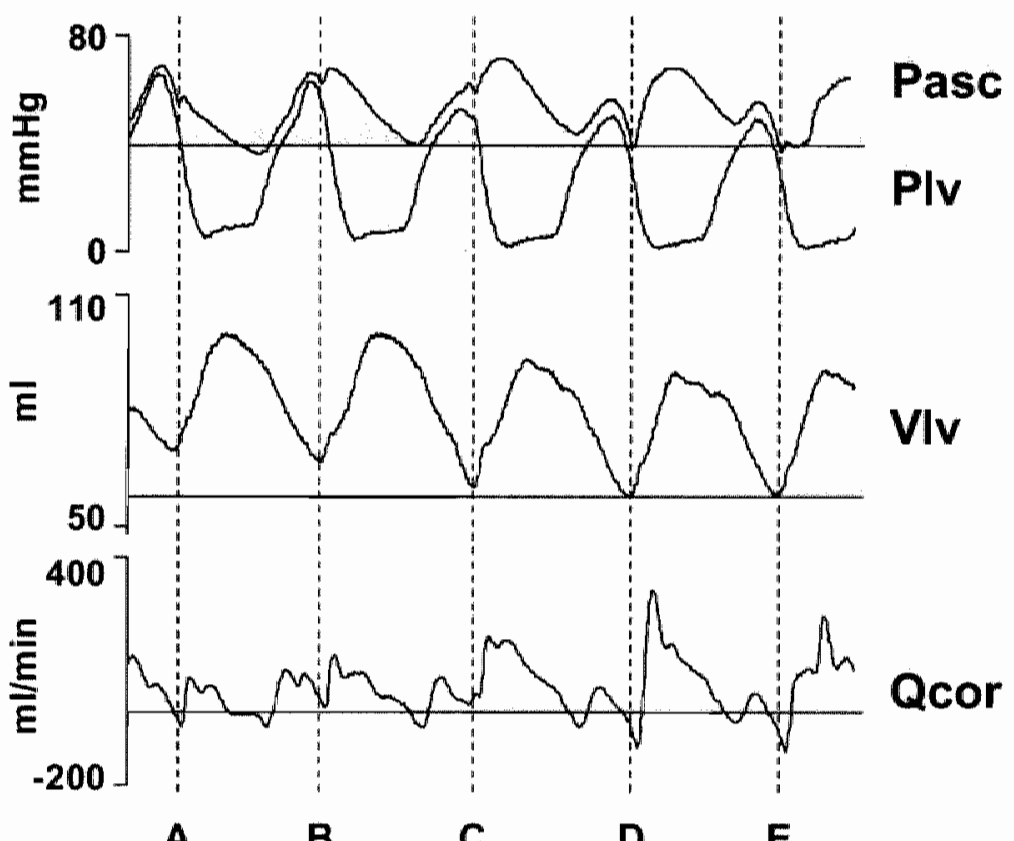

Figure 3.2 Five consecutive cardiac beats with various coincidences of the pump pulse during asynchronous support. Pump rate is lower than heart rate. The vertical dotted lines indicate aortic valve closure (start of diastole). Pasc, ascending aottic pressure; $\mathrm{Plv}$, left ventricular pressure; Vlv, left ventricular volume; Qcor, coronary artery flow. 
Figure 3.2 shows a typical hemodynamic recording of asynchronous pECLS support. Aortic pressure becomes biphasic with the PECIS pressure pulse arriving in diastole (beats $C, D$, and $E$ ). Left ventricular pressure is higher when the pulse arrives at the aortic valve during systole (beats $A$ and $B$ ). Both systolic and diastollic left ventricular volumes are higher during these beats as well. The changes in the coronary artery flow pattern correspond to those in aortic pressure. Progressively from beat $\mathrm{A}$ to $\mathrm{E}$ systolic coronary flow is reduced while diastolic flow is augmented. Diastolic coronary flow peaks early in diastole, which is associated with the upward slope of the pECLS pressure pulse. Beats similar to type A were selected for analysis of systolic arrival (SA). Similarly, D-type beats were averaged for diastolic arrival (DA) data. Figure 3.3 illustrates both beat types and the corresponding left ventricular pressure-volume loops. With systolic arrival ventricular ejection is decreased and pressure development is higher. The ratio of external work over potential energy is greater with diastolic arrival.

Table 3.2 Acute phase-dependent effect of asynchronous pumping

\begin{tabular}{|c|c|c|c|}
\hline & units & $\mathrm{SA}$ & DA \\
\hline Qpump & $1 / \min$ & $2.6(0.8)$ & $2.4(0.6)$ \\
\hline LVO & $1 / \min$ & $3.5(2.2)$ & $4.5(2.4)$ \\
\hline $\mathrm{CO}$ & $\mathrm{l} / \mathrm{min}$ & $6.2(2.3)$ & $6.9(2.0)$ \\
\hline Plv & $\mathrm{mm} \mathrm{Hg}$ & $107(27)$ & $92(25)$ \\
\hline Pascpunk & $\mathrm{mm} \mathrm{Hg}$ & $111(29)$ & $104(22)^{\#}$ \\
\hline Pase & $\mathrm{mm} \mathrm{Hg}$ & $83(23)$ & $84(21)$ \\
\hline $\mathrm{Pcv}$ & $\mathrm{mm} \mathrm{Hg}$ & $8.8(4.3)$ & $8.9(4.3)$ \\
\hline $\mathrm{Pla}$ & $\mathrm{mm} \mathrm{Hg}$ & $8.8(2.8)$ & $8.3(2.7)^{35}$ \\
\hline$V \mid v_{1: D}$ & $\mathrm{ml}$ & $110(61)$ & $109(59)$ \\
\hline $\mathrm{V} \|_{\mathrm{v}} \mathrm{s}$ & $\mathrm{ml}$ & $79(45)$ & $69(42)$ \\
\hline Qcon: & $\mathrm{ml} / \mathrm{min}$ & $73(49)$ & $79(4.6)^{\text {新 }}$ \\
\hline PVA & $\mathrm{Nm}$ & $1.03(0.72)$ & $0.91(0.58)$ \\
\hline EW/PVA & $\%$ & $48(22)$ & $57(22)^{\text {竞 }}$ \\
\hline
\end{tabular}

Values are mean (SD), $\mathrm{n}=10 .{ }^{H} \mathrm{p}<.05$ versus $\mathrm{SA}$ (systolic arrival); $\mathrm{DA}$, diastolic arrival; Qpump, bypass pump flow; LVO, left ventricular output; $\mathrm{CO}$, total cardiac output; PlwPEAK, peak left ventricular pressure; PascPEAK, peak ascending aortic pressure; Pasc, ascending aortic pressure; Pcv, central venous pressure; Pla, left atrial pressure; Vlven, end-diastolic left ventricular volume; Vlves, end-systolic left ventricular volume; Qcor, coronary artery flow; PVA, total pressure volume area; EW/PVA, ventricular efficiency. 


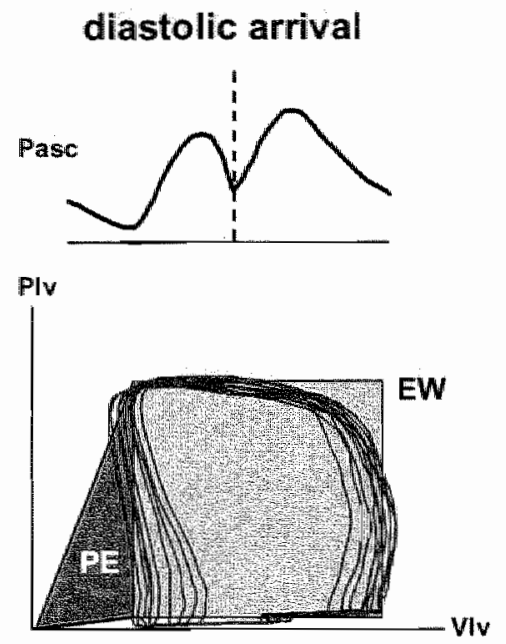

systolic arrival
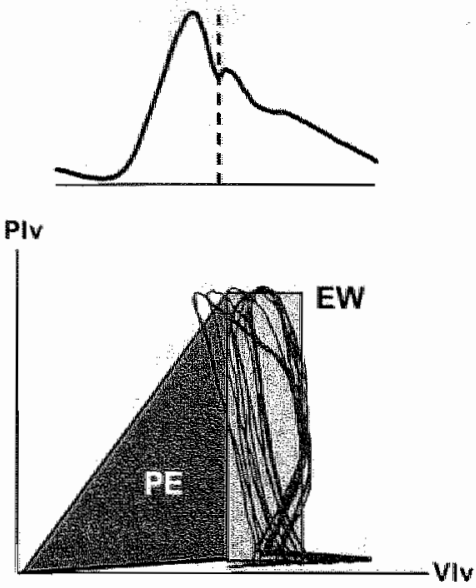

Figure 3.3 Pressure-volume (PV) data obtained during an episode of asynchronous support in which pump rate and heart rate were such, that every? other heart beat experienced systolic arrival (SA) following diastolic arrival (DA). Here, these pseudo-stable SA and DA loops atre depicted separately for the sake of PV area analysis. Top: Classification of asynchronous beats into DA and $\mathrm{SA}$ of the pump pulse. Bottom: Corresponding effect on left ventricular function. First order approximations of left ventricular external wotk (EW) and potential energy (PE) are shown within the pressure-volume planes. Pasc, ascending aortic pressure; $P \mathrm{lv}$, left ventricular pressure; Vlv, left ventricular volume.

\section{Asynchronous data}

Table 3.2 compares the hemodynamic and cardiac indices after differentiation of the phase of arrival. Compared to systolic arrival left ventricular output (LVO) is significantly higher during diastolic artival: SA, $3.5 \pm 2.2$ versus $\mathrm{DA}, 4.5 \pm 2.4 \mathrm{l} / \mathrm{min}$. Peak left ventricular pressure is significantly lower $(-14 \%)$ during $\mathrm{DA}$, but there is no significant difference in mean aortic pressure. Only end-systolic left ventricular volume is lower during $\mathrm{DA}(-13 \%)$, which amounts to an increase in ejection fraction from roughly $30 \%$ to $40 \%$. Mean coronary artery flow is slightly larger during DA than during SA. Total pressure volume area (PVA) was $12 \%$ smaller during 
DA (0.91 versus SA: $1.03 \mathrm{~N} \cdot \mathrm{m})$. Ventricular efficiency (EW/PVA) proved to be about $10 \%$ lower during systolic arrival of the pECLS pulse $(\mathrm{p}<.05)$.

\section{Synchronous evaluation}

In Figure 3.4 the results of synchronized pumping have been illustrated. The graph shows the sensitivity to pulse arrival of four determinants of myocardial working conditions in two individual subjects. The data was processed as follows: The $\mathrm{Plv}_{\mathrm{PEAK}}$ values of all delay settings were averaged. The delay setting corresponding with this average afterload was selected as the benchmark setting. The relative changes with respect to this benchmark are plotted on the vertical axis (Fig. 3.4). For instance, in the 100\% bypass case the Qcor trend shows only positive values: All delay settings produced an increase in coronary flow with respect to the coronary flow at the average afterload setting. The benchmark setting may be regarded as representative of non-synchronized pECLS or even continuous flow ECLS.

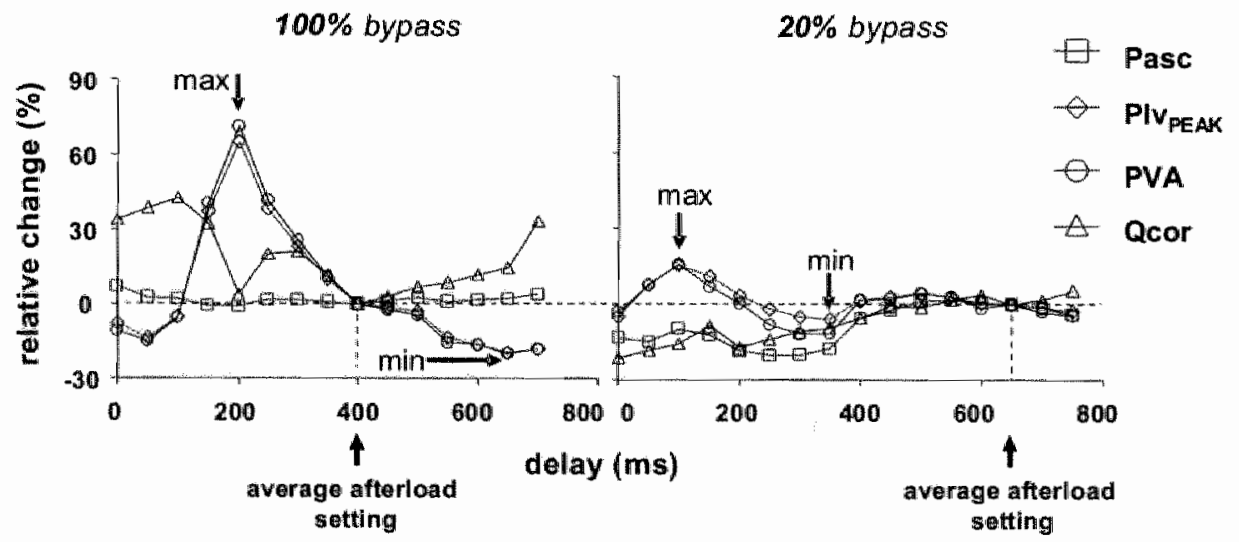

Figure 3.4 Synchronous evaluation of pump pulse arrival: Two distinct examples of bypass flow ratio are presented. A delay of $0 \mathrm{~ms}$ complies with the simultaneous ejection of blood from the left ventricle into the aorta and from the pump into the femoral artery. Every subsequent step on the horizontal axis adds another $50 \mathrm{~ms}$ to the delay in cardiac ejection. "The vertical axis represents the relative change of each variable with respect to the average afterload setting (left, at $400 \mathrm{~ms}$; right, at $650 \mathrm{~ms}$ ). For each example the maximum and minimum afterload levels are indicated. Pasc, mean ascending aortic pressure; Plvpsak, peak left ventricular pressure; PVA, pressure-volume area; Qcor, coronary artery flow. 
In the fully bypassed animal, manipulation of the timing of pECLS pulse incidence produced marked changes in peak left ventricular pressure, pressure-volume area (PVA), and coronary flow (Fig: 3.4). At the point of maximum afterload, PVA was increased by $70 \%$ while coronaty flow was not enhanced $(<5 \%)$. However, coronary flow increased more than $30 \%$ with pulse arrivals corresponding to minimum afterload and decreased PVA $(-20 \%)$. Ascending aortic pressure was only marginally affected by the variation in pullse arrival. In the low bypass case (Fig. 3.4, right panel) both negative and positive deviations do not exceed $15 \%$. Furthermore, a less strong correlation between the indices is noted.

In Table 3.3 the grouped hemodynamic differences between the afterload extremes have been summarized ( $n=5$ ). At maximum afterload, marked increases in left ventricular volume $(+35 \mathrm{mll})$ and PVA $(57 \%)$ were measured. Concomitantly, the diastolic coronary fraction and the ventricular efficiency showed a strong decline: DCF decreased from 93 to $53 \%$, and EW/PVA was compromised ( $28 \%$ versus $45 \%$ at minimum load).

Table 3.3 Hemodynamic indices at both extremes of $\mathbb{L V}$ afterload obtained with synchronized measurements.

\begin{tabular}{cccc}
\hline & units & Maximum afterload & Minimum afterload \\
\hline Plvesk & $\mathrm{mm} \mathrm{Hg}$ & $70(36)$ & $45(35)$ \\
Pasc & $\mathrm{mm} \mathrm{Hg}$ & $51(23)$ & $46(20)$ \\
Qpump & $1 / \mathrm{min}$ & $2.0(0.4)$ & $1.8(0.4)$ \\
LVO & $1 / \mathrm{min}$ & $1.9(4.2)$ & $1.7(3.9)$ \\
VlveD & $\mathrm{ml}$ & $123(69)$ & $89(78)$ \\
Vlves & $\mathrm{ml}$ & $96(41)$ & $61(49)$ \\
Qcot: & $\mathrm{ml} / \mathrm{min}$ & $33(36)$ & $31(41)$ \\
DCF & $\%$ & $53(12)$ & $93(19)$ \\
PVA & $\mathrm{N} \mathrm{m}$ & $0.74(1.0)$ & $0.47(0.81)$ \\
EW/PVA & $\%$ & $28(22)$ & $45(27)$ \\
\hline
\end{tabular}

Values are mean (SD), $n=5$. PlvpEak, peak left ventricular pressure; Pasc, ascending aortic pressure; Qpump, bypass pump flow; LVO, left wentriculat output; $\mathrm{CO}$, total cardiac output; VlvED, end-diastolic left ventricular volume; Vlves, end-systolic left ventricular volume; Qcor, coronary artery How; DCF, diastolic coronary flow; PVA, total pressure volume area; EW/PVA, wentricular efficiency. 
$56 \mid$ Chapter 3

\section{DISCUSSION}

Intra-aortic counterpulsation during non-pulsatile ECLS is commonly employed to address specific myocardial demands. However, the use of IABP during ECLS of CPB has not been firmly protocolized, and the effects on myocardial recovery have only been investigated in limited applications ${ }^{20}$. Most clinical investigations only report the use of conjunct IABP with ECLS without further specification and alledgedly ascribe favorable outcomes to counterpulsation and pulsatile perfusion 1,5,21,22. In the present study we focused on the potential of pulsatile ECLS systems to address myocardial support requirements.

The synchronous evaluation of pECLS indicated a pronounced effect of pulse incidence on ventricular function. Spot on systolic arrival (SA) was unambiguously associated with the highest left ventricular afterload (Fig. 3.4). Systolic impairment clearly compromises ventricular efficiency and the oxygen demand-supply balance (Table 3.3). Diastolically phased pECLS featured decreased LV afterload and oxygen demand, increased diastolic coronary perfusion, and improved ventricular efficiency (Fig. 3.4 and Table 3.3). Other investigators have found similar attributes under experimental conditions as well as in clinical practice ${ }^{1-3,5,23-26}$. Corday et al, showed that veno-arterial pulsatile partial bypass had greater efficacy in augmenting (diastolic) coronary flow than IABP alone ${ }^{23}$. Moreover, their setup incorporated additional end-diastolic arterial suction as a means to actively reduce left ventricular afterload. However, they found that in experiments in dogs as well as in selected clinical cases, active afterload reduction by pulsatile ECLS was associated with elevated renal flow resistance and morbidity ${ }^{24}$. Synchronously counterpulsating pECLS as presented in this paper does not actively reduce afterload.

Asynchronous PECLS support produced an increase in both coronaty and systemic perfusion pressure. The observed peak aortic pressure exceeded peak left ventricular pressure (Table 3.1). Apparently, the greater number of PECIS pulses arrived at the aortic valve during diastole. This could have been expected because diastole takes up more than $50 \%$ of the cardiac cycle period ${ }^{27}$. If we consider the effect well-aligned diastolic 
arrival (DA) pulses have (type D; Fig. 3.2), an enhanced aortic to ventricular pressure difference is noted (104 over $92 \mathrm{~mm} \mathrm{Hg}$ mean; Table 3.2). The DA data from the asynchronous protocol thus serve as a model of pulsatile ECLS, as if it wete phased to the diastolic period. However, these analyses depend on specific beat selections from asynchronous recordings, and therefore only describe the acute, psendo steady-state differences between $\mathrm{SA}$ and $\mathrm{DA}$.

\section{Oxygen demand and supply}

Mechanical cardiac support and specifically myocardial preservation are aimed at creating favorable myocardial working conditions. To achieve this, either a reduction in work (demand) or an augmentation of coronary flow (supply), or both are mandatory $18,21,25,28-30$. The results of the present study showed no dependence of mean coronary flow on pulse incidence. The insensitivity of mean coronary flow to these changes in perfusion pressure has been found in other studies, and is associated with intact coronary autoregulation ${ }^{23,25,26}$. Nonetheless, diastolic coronary perfusion did change dramatically with varying delays of synchronized ejection (Fig. 3.2, Table 3.3). The perfusion of subendocardial layers is known to depend strongly on diastolic flow ${ }^{21,27,31,32}$. To satisfy metabolic demands during partial bypass, the heart entirely depends on coronary flow reserve which normally is limited to $20-30 \%{ }^{11,27}$. This suggests that in pathological situations little headroom is left to fulfill myocardial oxygen requirements ${ }^{27,29}$.

Left ventricular myocardial work was significantly affected by systolic arrival of the pECLS pulse (Tables 3.2 and 3.3, Figs. 3.3 and 3.4). Pressure-volume area has been identified as an appropriate measure of myocardial oxygen consumption ${ }^{18}$. Avoidance of systolic impairment provided a reduction of $57 \%$ in oxygen consumption (Table 3.3). This capability to unload the myocardium while maintaining coronary flow and increasing subendocardial perfusion proves that synchronously counterpulsating pECLS is an interesting cardiac assist option when regarding myocardial working conditions. Failure to establish proper myocardial oxygen balance during ECLS may lengthen the therapeutic time 
path or may even lead to expansion of the ischemic/stunned myocardial mass 20,21,31. Pappas et al, studied the use of intra-aortic balloon pumping (IABP) during cardiopulmonary bypass in both low-risk and high-risk patients ${ }^{20}$. They found an immediate postoperative improvement of $\mathrm{LV}$ ejection fraction for the CPB plus IABP group. In the CPB only group, however, postoperative low output syndrome occurred and inotropic support and diuretics were necessary. Metabolic evaluations showed significantly higher myocardial lactate production in the CPB only group. Apparently, IABP support during CPB improved the myocardial oxygen balance, which resulted in better outcome.

\section{Alternative to standard ECLS?}

The implantation and management of both ECLS and IABP systems make the combined therapy invasive, (logistically) complex, and costly. Synchronously counterpulsating pECLS may combine the attributes of IABP with those of extracorporeal partial bypass in a single system. The augmentation of cardiac output and aortic pressure, and the simultaneous reduction of LV afterload suggest that myocardial oxygen consumption and peripheral perfusion pressure can be decoupled by phasing pECLS ejection into diastole (Tables 3.2 and 3.3, Figs. 3.3 and 3.4). In contrast, with nonpulsatile ECLS left ventricular afterload is directly related to bypass flow

which should fit the perfusion demand of all systemic organs $1,2,5,20$. This finding implies, that phased ejection pECLS has an advantage over continuous flow ECLS. The potential benefit of synchronously counterpulsating PECIS over continuous How ECLS plus IABP in terms of economy and simplicity remains to be investigated, preferably in a comparative (pre-) clinical trial.

The pump system used in this investigation was not equipped for synchronous support. Further technological developments in this direction should include: A sync input (ECG), a selectable delay option, and dedicated monitoring of aortic pressure. The initial setup and calibration may be facilitated by catheterization or $2 \mathrm{D}$-echo. The adjustment procedure of the pump ejection delay would be similar to that with ordinary intra- 
aortic balloon pumping (IABP) ${ }^{26,33}$. Due to the inertia inherent in the current pump design beat-to-beat adaptation of ejection timing may not be feasible; IABP features faster pumping technology that may cope better with rapid adjustments. In most clinical applications timing optimization over several cardiac beats would probably suffice and should be aimed at avoiding spot on systolic impairment (Fig. 3.4).

\section{Clinical context}

Recognizing the potential of phased ejection pECLS, it is important to identify where the advantages of this support modality fit in the therapeutic timeline. The patient suffering from reversible cardiogenic shock will be considered as a model in the following discussion ${ }^{2,3,29,31}$. In the early stages of treatment veno-arterial bypass flow will be near $100 \%$ of the total cardiac output and the minimal volume loading of the heart will ensure low absolute myocardial oxygen requirement allowing for stabilization of basal metabolism. However, in compromised areas of the myocardium the oxygen balance remains crucial, as pressure work still requires sufficient oxygen delivery (Figs. 3.3 and 3.4) ${ }^{18,28}$. Stunned myocardium would be the most vulnerable in this stage of support ${ }^{29,31,32}$. As soon as global systemic organ function has improved up to an acceptable level, cardiopulmonary activity should be increased as a first step in rehabilitation ${ }^{34,35}$. This second stage involves lower assist ratios and consequent increased myocardial loading ${ }^{1,28}$. Synchronously counterpulsating pECLS can be particularly helpful to optimize myocardial working conditions, and control these independently from global perfusion (Table 3.2 and 3.3, Fig. 3.4). The presence of working myocardium and increased coronary perfusion make this stage apt for the launch of adjunctive therapies ${ }^{32,36}$. When further progress is made towards weaning off ECIS, hemodynamic challenges can be employed to study cardiac and overall circulatory functional stability. Phased pECLS offers an alternative to specifically evaluate cardiac function by a simple switch over to the asynchronous mode or even timed systolic impairment for brief trial periods ${ }^{37}$. In later stages additional exercise testing should be employed for a more intensive evaluation of exercise 
$60 /$ Chapter 3

capacity ${ }^{35,38}$. Eventually, actual weaning can take place and the patient can be treated off mechanical support or bridged to transplantation or an assist device ${ }^{13,25,37}$.

\section{Study limitations}

Left ventricular function with synchronized pECLS was studied in five animals only. Solid statistical evidence could therefore not be produced, but these results did however prove consistent with the rest of the data and peer findings $5,20,23,24$.

The extracorporeal circuit setup we used had a limited capability in terms of pump rate $(\max .80 \mathrm{bpm})$ and flow $(1.9 \pm 0.3 \mathrm{l} / \mathrm{min})$. This was due to the small size of the cannulae and the limited filling time - both appear to be inherent to the pulsatile ECLS concept. The drainage of venous blood was essentially passive and depended on the gravitational effect and the elastic recoil of the TPLS pumping chamber only. In this study we chose to straightforwardly evaluate the properties of the TPLS system as a unit. In clinical practice, drainage may be enhanced by the use of kinetic or vacuum assisted drainage techniques ${ }^{39}$.

A representative model of cardiac failure was not included in this study's design, as our focus was on the mechanical interaction of pECLS with the left ventricle. The results show that bypass flow ratio is a key determinant of how much impact pulse arrival has on ventricular function. A full range of possible bypass situations was covered in this investigation, which proved useful in determining in which stage of clinical application synchronization would pay off. We believe that the basic findings of this study can be extrapolated to pathological conditions and associated assist modes. 


\section{Conclusion}

Asynchronous pulsatile extracorporeal life support (pECIS) may be modified to enhance myocardial working conditions regardless of systemic perfusion by phasing pump ejection into cardiac diastole. Synchronously counterpulsating pECLS provides an oportunity to optimize current ECLS practice.

\section{Acknowledgement}

This study was supported financially by the NewheartBio Corporation, Seoul, Korea. The authors want to express their gratitude to Mr. T. van der Nagel and Ms. A.C.M. Soemers for their support and effort.

\section{References}

1 Doll N, Kiaii B, Borger M, et al. Five-Year results of 219 consecutive patients treated with extracorporeal membrane oxygenation for refractory postoperative cardiogenic shock. The Annals of Thoracic Surgery 2004; 77:151-157

2 Bartlett RH, Rolloff DW, Custer JR, et al. Extracorporeal life support: the University of Michigan experience. Jama 2000; 283:904-908

3 Pagani FD, Aaronson KD, Swaniker F, et al. The use of extracorporeal life support in adult patients with primary cardiac failure as a bridge to implantable left ventricular assist device. The Annals of Thoracic Surgery 2001; 71:577-881

4 Leung W-H. Coronary and circulatory support strategies for percutaneous transluminal coronary angioplasty in high-risk parients. American Heart foumal $1993 ; 125: 1727-1738$

5 Murashita T, Eya K, Myatake T, et al. Outcome of the perioperative use of percutaneous cardiopulmonary support for adult cardiac surgery: factors affecting hospital mortality. Artif Organs 2004; 28:189-195

Wright $G$. Mechanical simulation of cardiac function by means of pulsatile blood pumps. J Cardiothorac Vasc Anesth 1997; 11:299-309

7 Taylor $\mathrm{K}$ M, Bain WH, Davidson KG, et al. Comparative clinical study of pulsatile and non-pulsátile perfusion in 350 consecutive patients. Thorax 1982; 37:324-330 Hickey PR, Buckley M], Philbin DM. Pulsatile and nonjoulsatile cardiopulmonary bypass: review of a counterproductive controversy. Ann Thorac Surg 1983; $36: 720-737$ 
9 Doughery $F$, Donovan $F$, Townsley M. Harmonic analysis of perfusion pumps. Journal of Biomedical Engineering 2003; $125: 814-822$

10 Gourlay T, Ballaux PK, Draper ER, et al. Early experience with a new technique and technology designed for the study of pulsatile cardiopulmonary bypass in the rat. Perfusion 2002; 17:191-198

11 Stanley $T$, Bailey $P$, eds. Anesthesiology and the cardiovascular patient. Dordrecht: Klluwer Acadenic Publishers, 1996

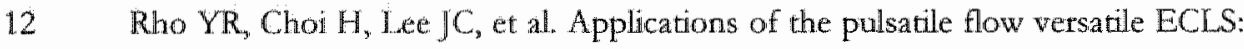
in vivo studives. Int J Artif Organs 2003; 26:428-435

13 Lee HS, Rho YR, Hwang CM, et al. In vivo ewaluation of the pulsatile ECLS system. J Artif Organs 2003; 6:25-29

14 Anderson HL, 3rd. Extracorporeal life support for cardiorespiratory failure. Adw Surg 1997: 31:189-215

15. Steendijk ?", Van der Velde ET, Baan J. Left ventricular stroke volume by single and dual excitation of conductance catheter in dogs. Am J Physiol 1993; 264:H2198-2207

16. Baan J, Van der Velde ET, De Bruin HG, et al. Continuous measurement of left ventricular volume in animals and humans by conductance catheter. Circulation $1984: 812-823$

17 Amirhamzeh MM, Dean DA, Jia $C X$, et al. Validation of right and left ventriculat conductance and echocardiography for cardiac function studies. Ann Thorac Surg $1996 ; 62: 1104-1109$

18 Suga $H$, Hayashi T, Shirahata M. Ventricular systolic pressure-volume area as predictor of cardiac oxygen consumption. Am J Physiol 1981; 240:H39-44

19 Suga $H$, Goto $Y$, Kawaguchi $O$, et al. Ventricular perspective on efficiency. BasicRes-Cardiol 1993; 88:43-65

20) Pappas $G$, Winter SD, Kopriva Cl, et al. Improvement of myocardial and other vital organ functions and metabolism with a simple method of pulsatile flow (IABP) during clinical cardiopulmonary bypass. Surgery 1975; 77;34-44

21 Mueller HS. Role of intra-aottic counterpulsation in cardiogenic shock and acute myocardial infarction. Cardiology 1994; 84:168-174

22 Phillips SI, Zeff RH, Kongtahworn C, et al. Benefits of combined balloon pumping and percutaneous cardiopulmonary bypass. Ann Thorac Surg 1992; 54:908-910

23 Corday E, Swan HJ, Lang TW, et al. Physiologic principles in the application of circulatory assist for the failing heart. Intraartic balloon circulatory assist and venoarterial phased partial bypass. Am I Cardiol 1970; 26:595-602

24 Lang TW, Rosselor E, Gold H, et al. Effect of wenoarterial pulsatile partial bypass on the coronary, renal and mesenteric circulations in cardiogenic shock. Am I] Cardiol 1971:27:41-45 
25 Reesink $\mathrm{KD}$, Dekker $\mathrm{AL}$, Van Ommen V, et al. Miniature intracardiac assist device provides more effective cardiac unloading and circulatory support during severe left heart fallure than intraaotic balloon pumping. Chest $2004 ; 126896-$ 902

26. Dekker Al., Reesink KD, van der Veen FH, et al. Intra-aortic balloon pumping in acute mitral regurgitation reduces aorric impedance and regurgitant fraction. Shock 2003; 19:334-338

27 Guyton AC. Medical Physiology. Gth ed. Philadelphia: W.B. Saunders Co, 1981

28 Suga $\mathrm{H}$, Hisano $\mathbb{R}$, Hirata $\mathrm{S}$, et all. Mechanism of higher oxygen comsumption rate: pressure-loaded vs. volume-loaded heart. Am J Physiol 1982; 242:H942-948

29 Hollenberg SM, Kavinsky CJ, Partillo JE. Cardiogenic shock. Ann Intern Med $1999 ; 131: 47-59$

30 Dekker A, Reesink $K$, van der Veen E, et al. Efficacy of a new intraontic propeller pump vs the intraaortic balloon pump: an animal study. Chest 2003; 123:2089-2095

31 Mangano DT. Myocardial stunning: an overview. J Card Surg 1993; 8:204-213

32 Kloner RA, Rezkalla SH. Cardiac protection during acute myocardial infarction: Where do we stand in 2004? Joumal of the American College of Cardiology 2004; 44:276-286

33 Kantrowitz A, Cardona RR, Freed PS. Percutaneous intra-aortic balloon counterpulsation. Crit Care Clin 1992; 8:819-837

34 Hon JK, Yacoub MH. Bridge to recovery with the use of left ventricular assist device and clenbuterol. Ann Thorac Surg 2003; 75:\$36-41

35 Sakuragi $S$, Takagi S, Suzuki S, et al. Patients with large myocardial infarction gain a greater improvement in exercise capacity after exercise training than those with small to medium infarction. Clin Cardiol 2003; 26:280-286

36 Chachques J, Cattadori B, Herreros J, et al. Treatment of heart failure with autologous skeletal myoblasts. Herz 2002; 27:570-578

37 Colson P, Ryckwaet F, Saussine M, et al. Monitoring weaning from BIVAD Thoratec with peak oxygen consumption. Ann Thorac Surg 2004; 77:1808-1810

38 Gordon NF, Gulanick M, Costa F, et al. Physical Activity and Exercise Recommendations for Stroke Survivors: An American Heart Association Scientific Statement From the Council on Clinical Cardiology, Subcommittee on Exercise, Cardiac Rehabilitation, and Prevention; the Council on Cardiovascular Nursing; the Council on Nutrition, Physical Activity, and Metabolism; and the Stroke Council. Circulation 2004; 109:2031-2041

39 Toomasian JM, McCarthy JP. Total extrathoracic cardiopulmonary support with kinetic assisted venous drainage: experience in 50 patients. Perfusion 1998; 13:137-143 
64|Chapter 3 


\section{Chapter 4}

\section{The acute effect of intra-aortic balloon counterpulsation during extracorporeal life support}

\section{Submitted as:}

Sauren LDC, Reesink KD, Selder JL, van der Nagel T, de Jong MMJ, Beghi C, van der Veen $F H$, Maessen $\ G$. The acute effect of intra-aortic balloon counterpulsation duting extracorporeal life support. 


\section{ABSTRACT}

\section{Introduction}

About one percent of patients undergoing cardiac surgery and patients suffering from hemodynamic instability after unsuccessful PTCA do not respond to intra-aortic balloon pump (IABP) therapy. Maintenance of hemodynamic stability in those patients can be obtained by the use of extracorporeal life support (ECLS). However, support of the failing heart might be improved by combining ECLS and IABP.

\section{Methods}

Seven sheep were supported by an IABP and an ECLS system which was cannulated centrally (CC) as well as peripherally (PC). In each cannulation configuration hemodynamic indices were measured at baseline, ECLS, and ECLS plus IABP. The major output parameters were myocardial oxygen supply (coronary flow (Qcor), diastolic pressure time index (DPTI)) and left ventricular oxygen demand (pressure volume area (PVA), tension time index (TTI)).

\section{Results}

The use of IABP on top of ECLS (CC/PC) significantly decreased left ventricular afterload (Plv,peak $-4 \% /-8 \%$ ), as well as TTI $-2 \% /-10 \%$ and PVA $-10 \% /-12 \%$. Coronary and endocardial perfusion were significantly increased by additional IABP; CC: Qcor $+9 \%$ and DPTI $+18 \%$, and PC: Qcor $+6 \%$ and DPTI $+11 \%$. IABP augmented the myocardial oxygen supply/demand ratios (CC/PC): Qcor/PVA (+21\%/22\%) and DPTI/TTI $(27 \% / 24 \%)$.

\section{Canciurion}

In both ECLS cannulation settings the additional use of IABP improves the balance between myocardial oxygen supply and oxygen demand. Myocardial recovery may be enhanced by IABP complementary to ECLS support. 


\section{INTRODUCTION}

The intra-aortic balloon pump (IABP) is the routine cardiac support device after acure myocardial infarction, postcardiotomy myocardial dysfunction, and cardiogenic shock ${ }^{1,2}$. However, about one percent of patients undergoing cardiac surgery and patients suffering from hemodynamic instability after unsuccessful PTCA will not respond to IABP therapy ${ }^{3,4}$. Intra-aortic balloon counterpulsation reduces left ventricular afterload and increases coronary flow, but has a minimal effect on cardiac preload and cannot independently support the systemic circulation in case of low cardiac output syndrome 1,5. Maintenance of hemodynamic stability in those patients can be obtained by the use of extracorporeal life support (ECLS). Depending on the circumstances of the patient, two veno-arterial cannulation configurations of the ECLS system are employed, namely central cannulation (CC) and peripheral cannulation (PC). Regardless of the cannulation configuration, during ECJ.S support a rise in pressure loading of the left ventricle is inevitable. The combination of the two support mechanisms of IABP and ECLS may result in an improved efficacy of the cardiac support as a whole.

The combination of IABP with other assist devices has been examined before ${ }^{1,6-13}$. Improved weaning rates and myocardial recovery were reported after the use of IABP during ECIS support ${ }^{1,8-10}$. However, the additional benefit of balloon counterpulsation has not been studied in detail yet. The purpose of this investigation in sheep was to examine the hemodynamic effect of intra-aortic balloon counterpulsation during extracorporeal life support in both the peripheral and central cannulation settings.

\section{METHODS}

\section{Animal preparation'}

All animals received humane care in compliance with the "Guide for the care and use of laboratory animals" of the National Institutes of Health. 
68 Chapter 4

The study was approved by the animal ethics committee of the University of Maastricht. Seven sheep (mean $\pm S D, 85 \pm 11 \mathrm{~kg}$ ) were premedicated with atropine $(0.05 \mathrm{mg} / \mathrm{kg}$ s.c.). Anesthesia was induced with sodium thiopental (i.v bolus $20 \mathrm{mg} / \mathrm{kg}$ ) and maintained with a $1: 2$ mixture of $\mathrm{O}_{2} \cdot \mathrm{N}_{2} \mathrm{O}$, and isoflurane (1.5\%). After administration of analgesic buprenorfine (i.v. bolus $0.01 \mathrm{mg} / \mathrm{kg}$ ) and muscle relaxant suxamethonium (i.v bolus $0.1 \mathrm{mg} / \mathrm{kg}$ ), a left thoracotomy was performed. Coagulation was controlled through administration of heparin (bolus $200 \mathrm{IU} / \mathrm{kg}$ i.v.) and monitored by activated clotring time (ACT) measurements. ACT was kept above 480 seconds during the experiment. Monitoring included ECG, blood pressure, oxygen saturation and capnography.

The animal was sacrificed through a pentobarbital overdose (bolus $80 \mathrm{mg} / \mathrm{kg}$ i.v.).

\section{Cardiac support systems}

The ECLS system was configured as a veno-arterial extracorporeal membrane oxygenation circuit and consisted of a centrifugal pump (MEDOS DP1, MEDOS, Aachen, Germany), an oxygenator and a venous reservoir. Two arterial cannulas (size 18-21F; Jostra, Hirrlingen, Germany) were inserted in each animal, one directly into the ascending aorta and one into the abdominal aorta via the left femoral artery. Venous drainage was achieved with a $21 \mathrm{~F}$ cannula inserted into the left femoral vein with placement of the tip in the right atrium.

Either central or peripheral arterial cannulation was selected by clamping one of the arterial cannulas. In both cannulation settings the bypass flow was gradually increased to a partial bypass configuration in which the heart and ECLS equally contributed to the total output (50-50\% setting).

A $40 \mathrm{ml}$ intra-aortic balloon catheter (Datascope, Fairfield, NJ) was introduced via the right femoral artery and placed in the high descending aorta. The intra-aortic balloon pump (System 98 XT IABP; Datascope, Fairfield, ND) was triggered by the ECG. 


\section{Instrumentation}

A conductance catheter (CD Leycom, Zoetermeer, the Netherlands) (which incorporated a pressure sensor), was positioned in the left ventricle via the left carotid artery ${ }^{14}$. The conductance catheter was connected to a Sigma 5DF conductance processor (CD Leycom, Zoetermeer, The Netherlands) which was used in dual-field mode. Solid-state pressure catheters (CD Leycom, Zoetermeer, The Netherlands) were placed in the ascending aorta via a $Y$-connector, which was placed just upstream of the arterial cannulae and in the left atrium through a purse-string suture. Ultrasonic flow probes (Transonic Systems, Ithaca, NY) were placed on the pulmonary artery, on a side branch of the left anterior descending coronary artery, and on the ECLS arterial outflow. All hemodynamic data were acquired and stored using a 16-channel acquisition system (Conduct-PC, CD Leycom, Zoetermeer, The Netherlands) and a 32-channel acquisition system (IdeeQ 1.70, Instrument Development Engineering \& Evaluation, University of Maastricht, the Netherlands).

\section{Conductance callibration}

Parallel conductance was determined by injecting $7.5 \mathrm{ml}$ of $6.5 \%$ hypertonic saline into the pulmonary artery ${ }^{14}$. A $5 \mathrm{ml}$ blood sample was collected in a sampling cuvette for blood resistivity measurement (CD Leycom, Zoetermeer, the Netherlands) ${ }^{15}$. The gain calibration factor was determined by comparing conductance derived left ventricular output with the pulmonary artery flow (Qpa) at baseline. The conductance data was analysed with the Circlab 2004 software package (Paul Steendijk, Leiden University Medical Center, Leiden, The Netherlands).

\section{Measurements and analysis}

During the baseline measurements both cardiac assist devices were switched off. In each cannulation setting a fifty-fifty percent bypass flow mode was achieved. Five to ten minutes were taken in consideration to establish 
steady-state measurements. IABP support (1:1 assist mode) was tuned to maximum diastolic aortic pressure augmentation and left ventricular afterload reduction during partial ECLS support. The activation of IABP was repeated 2 times in each cannulation setting.

The hemodynamic measurements were analysed at baseline and support conditions to obtain indices of left ventricular function, myocardial working condition and peripheral perfusion. The diastolic pressure time index (DPTI) is a measure for diastolic coronary blood flow, which is calculated by the area between aortic pressure signal and left ventricular pressure signal in diastole ${ }^{16-19}$. The tension time index (TTT) is the enclosed area under the left ventricular pressure curve and correlates with the left ventricular afterload 10,18,20. The ratio between the two parameters is characterised as the endocardial viability ratio DPTI/TTI $(\%){ }^{16}$. The total pressure-yolume arca (PVA) represents the total mechanical work per heart beat ${ }^{21-23}$. The enclosed pressure-volume area consist of left ventricular external work (EW) and mechanical potential energy (PE) ${ }^{22.24}$ and was estimated from: $E W=($ Vlv,ed - Vlv,es $) \cdot($ Plv,peak - Plv,ed $)$ and $P E=0.5$ - Vlv,es $\cdot(\mathrm{Plv}$, peak - Plv,ed); total pressure volume area PVA $=E \mathrm{EW}+\mathrm{PE}$. The Qcor/PVA ratio was calculated and should be interpreted as the volume of arterial coronary blood available per unit of ventricular work, per heart beat.

Statistical comparisons (Wilcoxon signed-ranks test, $\mathrm{n}=7$ ) were made between baseline and ECLS support, and between ECLS and combined 'ECLS plus IABP' support. The relative effect (in \%) of IABP in the central cannulation configuration is compared to the relative effect of IABP with peripheral cannulation.

\section{RESULTS}

Partial cardiopulmonary bypass was achieved upon institution of extracorporeal life support: The ECLS system produced an average flow of $2.8 \pm 0.9 \mathrm{l} / \mathrm{min}$ (mean $\pm \mathrm{SD}, \mathrm{n}=7$ ) while left ventricular output (LVO) was $2.8 \pm 1.0 \mathrm{l} / \mathrm{min}$. The hemodynamic effects of the various cardiac support systems are summarized in Table 4.1. Use of the ECLS system in both 
cannulation settings significantly increased ascending aortic pressure by $29 \%$ in central cannulation (CC) and by $26 \%$ in peripheral cannulation (PC). ECLS support significantly increased TTI (24\% (CC), 24\% (PC)) and DPTI (39\% (CC), 54\% (PC)). Both the DPTI/TTI and Qcor/PVA ratios did not change significantly by the use of ECLS support alone. Figure 4.1 provides an illustrative example of the acute effect of ECLS on left ventricular function; in the same diagram the additional effect of IABP is shown as well. Implementation of intra-aortic balloon counterpulsation during ECLS support (CC/PC; Table 4.1) resulted in a significant reduction of left

Table 4.1 The hemodynamic effect of IABP during partial ECLS support:

\begin{tabular}{|c|c|c|c|c|c|c|}
\hline & & \multirow[b]{2}{*}{ Baseline } & \multicolumn{2}{|c|}{ Central cannulation } & \multicolumn{2}{|c|}{ Peripheral cannulation } \\
\hline & & & ECLS & ECLS+1ABP & ECLS & ECLS+IABP \\
\hline Plwpeak & $\mathrm{mm} \mathrm{Hg}$ & $65(16)$ & $75(25)$ & $72(25)^{n}$ & $72(29)$ & $67(28)^{5}$ \\
\hline Pasc & $\mathrm{mm} \mathrm{Hg}$ & $52(10)$ & $68(24)^{*}$ & $72(23)^{t i}$ & $66(26)^{*}$ & $67(27)$ \\
\hline LVO & $1 / \mathrm{min}$ & $4.1(1.0)$ & $3.1(0.9)$ & $3.0(1.0)^{\# t}$ & $2.5(1.1)^{\text {k/ }}$ & $2.7(1.1)$ \\
\hline Qcor & $\mathrm{mm} / \mathrm{min}$ & $110(63)$ & $104(68)$ & $114(76)^{*}$ & $101(59)$ & $106(62)\}$ \\
\hline EW & $\mathrm{J} /$ beat & $0.33(0.13)$ & $0.30(0.13)$ & $0.27(0.12)^{3}$ & $0.24(0.11) *$ & $0.24(0.14) t$ \\
\hline PE & J/beat & $0.30(0.30)$ & $0.35(0.28)^{*}$ & $0.32(0.26)^{7}$ & $0.37(0.33)^{*}$ & $0.30(0.25)^{5}$ \\
\hline PVA & J/beat & $0.63(0.41)$ & $0.65(0.39)$ & $0.59(0.36)^{4}$ & $0.61(0.36)$ & $0.54(0.37)^{5}$ \\
\hline Qcor/ & & & & & & \\
\hline PVA & $\mathrm{ml} / \mathrm{d}$ & $2.04(1.32)$ & $1.78(1.23)$ & $2.15(1.52)^{\text {特 }}$ & $2.38(1.99)$ & $2.92(2.65)$ \\
\hline TTI & Pars & $16(6)$ & $20(8)^{*}$ & $18(8)^{\text {部 }}$ & $20(10)^{*}$ & $18(10)$ \\
\hline DPTI & Prit $_{\text {tits }}$ & $14(5)$ & $19(8)^{*}$ & $22(8)^{\text {ff }}$ & $22(10)^{x / 2}$ & $24(11)+$ \\
\hline DPTT/ & & & & & & \\
\hline TTI & 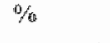 & $90(19)$ & $100(20)$ & $127(29)^{t t}$ & $110(23)$ & $136(27) 5$ \\
\hline
\end{tabular}

Mean (SD), statistical comparison by Wilcoxon signed-ranks test; $\mathrm{n}=7 . * \mathrm{p}<0.05$ ECLS versus baseline, ${ }^{2} \mathrm{p}<0.05$ ECLS + IABP versus ECIS alone, central cannulation, ${ }^{\$} \mathrm{p}<0.05$ ECLS + IABP versus ECLS alone, periphetal cannulation, $\dagger p<0.05$ effect of IABP? peripheral cannulation versus effect of IABP central cannulation. Plv,peak=left ventricular systolic peak pressure, $\mathrm{Pasc}=$ mean ascending aortic pressure, $\mathrm{LVO}=$ left ventricular output, Qcor=mean coronary artery flow. (All left ventricular indices:) EiW=external work, $\mathrm{PE}=$ potential energy, PVA=pressure volume area, TTI=tension time index, $\mathrm{DPTT}=$ diastolic pressure time index. 
ventricular peak pressure $(-4 \% /-8 \%)$ and of the tension time index $(-2 \% /-10 \%)$. Simultaneously, IABP reduced total left ventricular work (PVA) significantly by $-10 \%$ (CC) and $-12 \%(\mathrm{PC})$, which was primarily due to decreases in potential energy (PE) of $-10 \%$ and $-16 \%$ respectively. The latter effect is illustrated in Figure 4.1: the loop area (external work, EW) does not change much from ECLS to ECLS plus IABP, but the loop is shifted to the left on the volume axis.

Figure 4.2 shows an example of the counterpulsation effect on the ascending aortic and left ventricular pressure in both cannulation modes. The recordings were obtained from a single experiment. Additional IABP generated a significant rise in mean coronary artery flow ( $+9 \%(\mathrm{CC})$ and $+6 \%(\mathrm{PC}))$ and in the diastolic pressure time index $(+18 \%(\mathrm{CC})$ and $+11 \%$ (PC)). The increases in DPTI and Pasc due to IABP with centrally cannulated ECLS are respectively $8 \%$ and $6 \%$ higher than in peripheral mode (Table 4.1).

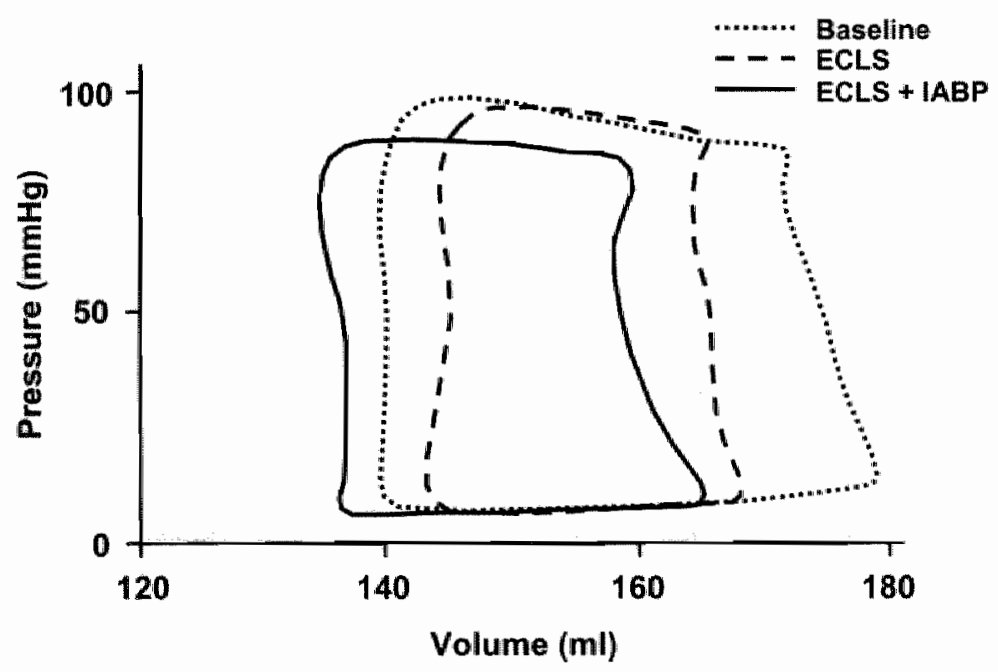

Figure 4.1 Acute effect of support on left ventricular (I.V) function. Pressurevolume data were obtained in one animal in petipheral cantulation mode. Extracorporeal life support (ECLS) support reduces LV stroke volume (loop is narrower with respect to baseline). Additional intra-aortic balloon pumping $(\mathrm{ABBP})$ reduces $\mathrm{L} V \mathrm{~V}$ afterload and mean $L V$ volume. 


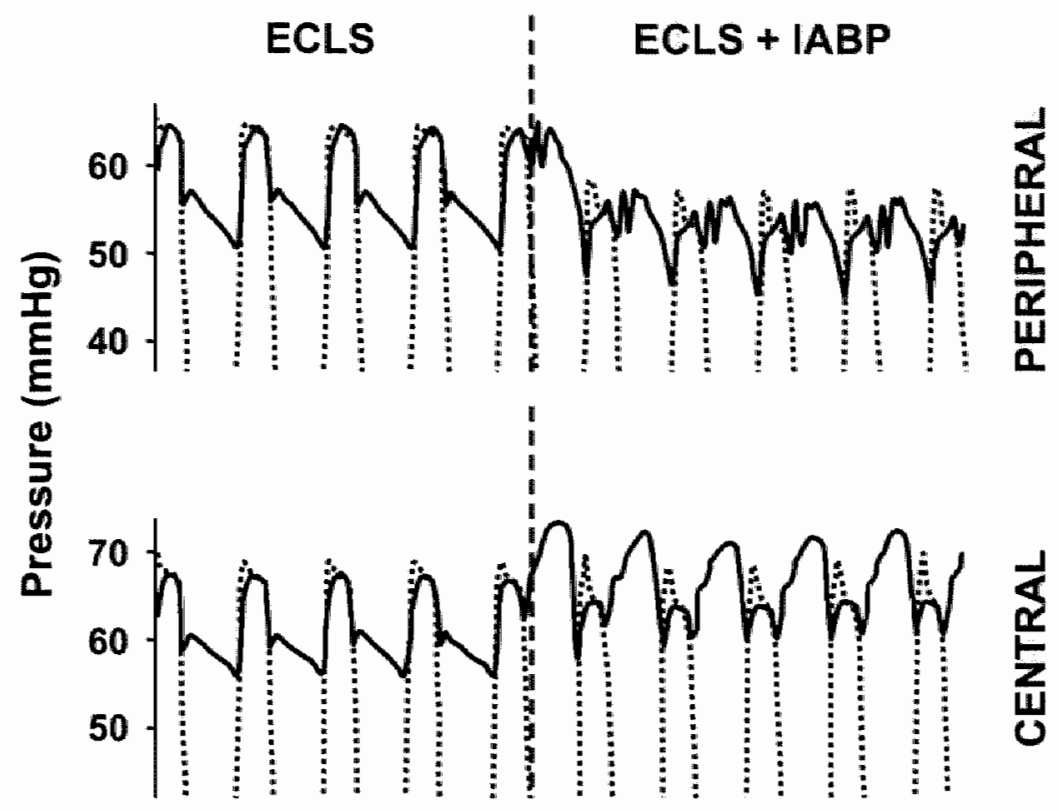

Figure 4.2 Recordings of the acute effect of intrawartic balloon pumping (IABP) on the ascending aortic and left ventricular pressures during extracorporeal life support (ECLS). Data was obtained from one animal. Both the central and peripheral cannulation setrings are shown. In peripheral mode, the diastolic augmentation of the IABP seems attenuated, but LV afterload is clearly reduced. "The reverse appears to apply with the centrally cannulated ECLS. 
The DPTI/TTI and Qcor/PVA ratios significantly changed with IABP during ECLS support (Table 4.1). With IABP the Qcor/PVA ratio increased by $21 \%$ with central cannulation, and by $22 \%$ in the peripheral setting. The endocardial viability ratio (DPTI/TTT) showed a similar rise, $27 \%$ (CC) and $24 \%$ (PC). The relationships between these balanced indices and their constituents can be appreciated from Figure 4.3.

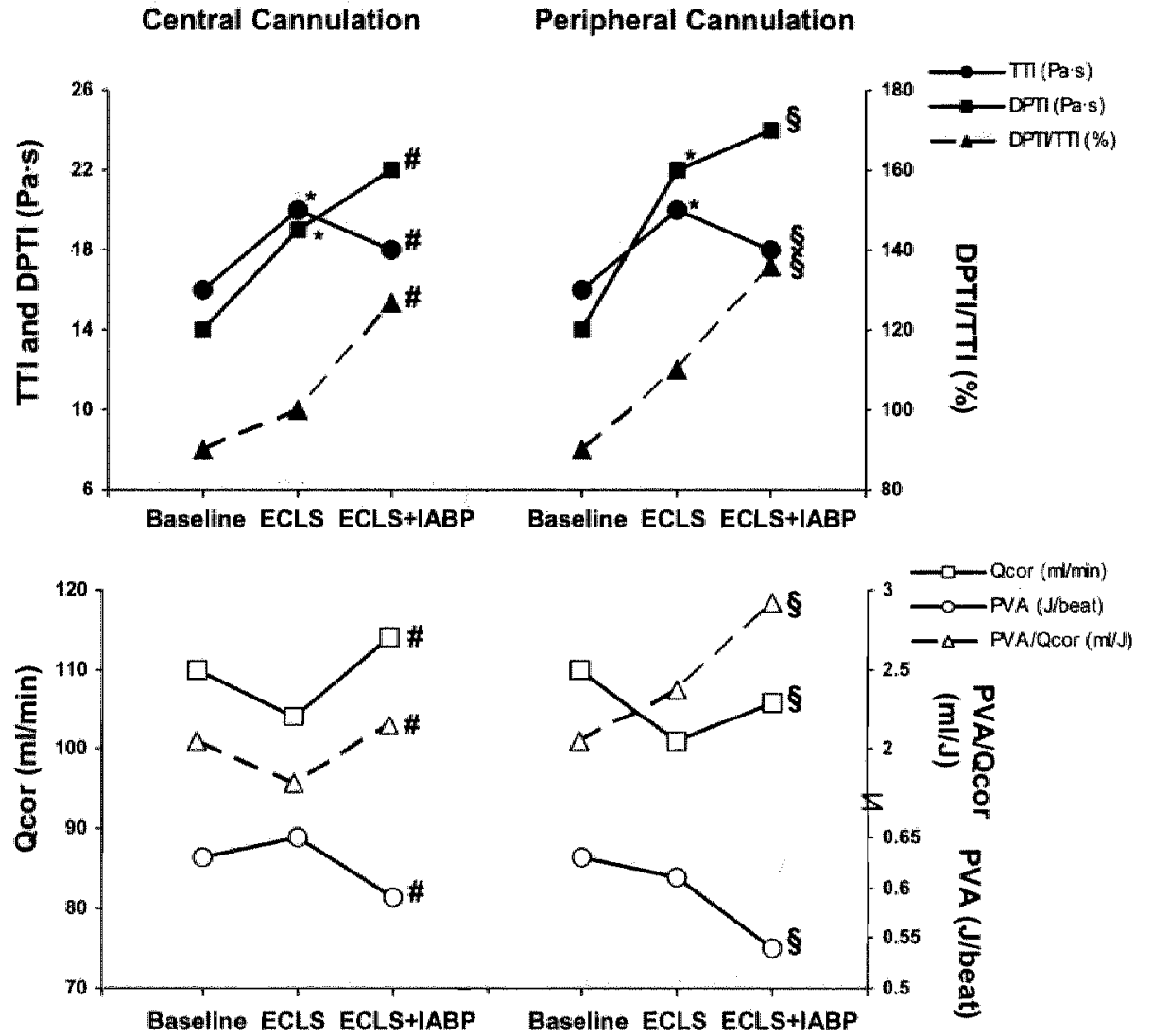

Figure 4.3 Changes in the coronary oxygen supply and demand at baseline, with ECLS, and with ECLS plus IABP support. Top: Tension time index (TTI), diastolic pressure time index (DPTI), and DPTI/"TTI. Bottom: Coronary flow (Qcor), total pressure volume area (PVA), and PVA/Qcor. Wilcoxon signed-ranks test, $\mathrm{n}=7$. ${ }^{*} \mathrm{p}<0.05$ ECLS wersus baseline, * $\mathrm{p}<0.05$ ECLS+IABP versus ECLS alone, central cannulation, $\$ p<0.05$ ECLS+IABP versus ECLS alone, peripheral cannulation. 


\section{DISCUSSION}

Theoretically, the combination of IABP counterpulsation and extracorporeal life support may be advantageous in terms of myocardial support. Several clinical studies claim a beneficial effect of additional IABP during ECLS support. These investigations concluded that the combined ECLS plus IABP support contributed to the most optimal recovery of acutely ischemic myocardium ${ }^{7,9}$ and appeared to be a better therapy than IABP or ECLS individually ${ }^{1,8}$. Although the combination therapy was recommended, the mechanism has not been completely understood ${ }^{8}$. In the present study, the mechanism is examined through hemodynamic evaluations with the main focus on the balance between myocardial oxygen supply and consumption.

\section{Previous studies}

The mechanism of intra-aortic balloon counterpulsation during ECLS support has been studied by other investigators, but none of them have addressed the different cannulation options with ECIS. Phillips et al examined only one hemodynamic parameter in the ECLS plus IABP combination support, namely mean arterial pressure ${ }^{1}$. The results illustrated that patients with combined support have a higher mean arterial pressure compared to ECLS support alone. Various hemodynamic parameters were measured by Bavaria et al in animals ". "These investigators studied the correlation between left systolic ventricular wall stress and oxygen consumption in both control and in combination support groups to explain the apparent benefit. Although the highest mean coronary artery flow was measured with the combined ECLS plus IABP support, this could not be attributed solely to the additional balloon counterpulsations. The most favourable setting concerning myocardial oxygen consumption and systolic wall stress was, however, not found in the combined ECLS plus IABP support configuration. Also no correlation between the two parameters could be found as these investigators had anticipated. Thus, no adequate explanation for the benefits of combined support could be specified so far. 


\section{Hemodynamic approach}

Our perspective to demonstrate the advantages of ECLS plus IABP support is based on the presumption that myocardial oxygen supply and myocardial oxygen consumption are the determining factors for myocardial recovery. Different indices are used to measure the myocardial oxygen supply and the myocardial oxygen consumption (or demand). We use the mean coronary artery blood flow and the diastolic pressure time index as indices for oxygen supply; the pressure volume area and the tension time index are used as indices for oxygen consumption (demand). These parameters are presented in Table 4.1 and Figure 4.3.

Assuming the arterial blood is fully saturated, the mean coronary artery flow (Qcor) correlates with the oxygen supply of the myocardium. This assumption is also required for the use of the diastolic pressure time index (DPTI) as an estimate of subendocardial oxygen supply ${ }^{19,25}$. Both oxygen supply indices (Qcor, DPTT) were augmented by additional intraaortic balloon counterpulsation during central as well as peripheral ECLS support.

Total left ventricular work, represented by the total pressure volume area (PVA), is linearly related to the myocardial oxygen consumption ${ }^{20-24}$. Another good quantitative estimation for oxygen requirement is the tension time index ${ }^{17}$. Both parameters indicate that the LV myocardial oxygen demand (PVA, TTI) is reduced by intra-aottic balloon counterpulsation during ECLS support in both CC and PC settings.

The additional use of IABP support with ECLS support increases the coronary perfusion and decreases left ventricular PVA, which implies an improvement in coronary oxygen balance. To focus on the myocardial working condition more specifically we introduced the balanced indices Qcor/PVA and the endocardial viability ratio (DPTI/TTI). LV tension time index, correlated with oxygen consumption, increases with ECLS support alone, but is compensated by the rise in coronary oxygen supply, causing the oxygen supply/demand balance to remain unaffected (Fig. 4.3). With additional IABP this balance does change. The established rise in oxygen supply and reduction in oxygen consumption in both cannulation 
configurations result in a significant increase in the myocardial oxygen supply/demand balance (Fig. 4.3). In both clinical and experimental settings, an improved myocardial oxygen supply/demand balance has been shown to promote recovery from reversible myocardial damage ${ }^{16,1826.31}$.

\section{Cannulation}

Two cannulation configurations are possible in a veno-arterial ECLS system, namely central cannulation and peripheral cannulation. Acute postoperative ECLS procedures most frequently employ the existing central configuration 8 , while most research has been performed in peripheral cannulation settings $1,9,11$. In the present study, LABP has been implemented under both cannulation conditions in order to investigate not only the hemodynamic mechanism of IABP in each mode, but also to determine whether that mechanism differs with respect to cannulation.

In the centrally cannulated ECLS, the imptovements with IABP of mean aortic pressure (Pasc) and the diastolic pressure time index (DPTI) are significantly higher compared to the effect in the peripheral configuration (Table 4.1, Fig. 4.2). Despite the fact that the above mentioned effects differ significantly, in none of the other indices any significant differences were noted. IABP retains its characteristic unloading and diastolic augmentation properties when used in combination with partial ECLS, regardless of the cannulation configuration. Under partial bypass conditions deviant from the $50-50 \%$ ratio the effect of adjunctive IABP may be different, but this has not been studied in the present study. The consistent improvement of the oxygen suppiy/demand balance implies that intra-aortic balloon counterpulsation may benefit any clinical use of ECLS in partial bypass mode.

\section{Clinical perspective}

When ECLS therapy is used as a bridge to recovery, full left ventricular unloading may not be the most optimal approach ${ }^{32-37}$. Partial loading of the heart is associated with improved ventricular function, myocardial blood 
flow, metabolism, and reduced infarct size ${ }^{36,37}$. In this hemodynamic evaluation a partial bypass ECLS mode was chosen to simulate a bridge to recovery setting.

When full recovery of the myocardium is anticipated, a weaning procedure is to be considered eventually. Long term ECLS is associated with major complications such as renal failure, lower limb ischemia, neurological complications, and infections ${ }^{3,4,7,8,38,39}$. Consequently, the timely disconnection of the ECLS system is desirable. Intermediate support provided by a less invasive assist device, like the IABP, may facilitate the early weaning from ECLS ${ }^{10,40}$.

\section{Conclusion}

The additional use of IABP with ECLS improves the myocardial oxygen balance, regardless of the cannulation configuration. Adjunctive IABP should therefore be considered in bridge to recovery indicated ECLS cases.

\section{Acknowledgement}

This study was supported by Medos (Aachen, Germany) and Datascope (Fairfield, NJ).

\section{References}

1 Phillips S1, Zeff R.H, Kongtahworn $C$, et al. Benefits of combined balloon pumping and percutaneous cardiopulmonaty bypass. Ann Thorac Surg 1992; $54: 908-910$

2 Hollenberg SM, Kavinsky CJ, Parrillo JE. Cardiogenic shock. Ann Intern Med 1999; $131: 47-59$

3 Muehrcke DD, McCarthy PM, Stewart RW, et al. Extracorporeal membrane oxygenation for postcardiotomy cardiogenic shock. Ann Thorac Surg 1996; $61: 684691$

4 Smith C, Bellomo R, Raman JS, et al. An extracorporeal membrane oxygenationbased approach to cardiogenic shock in an older population. Ann Thorac Surg $2001 ; 71: 1421-1427$ 
Murphy DA, Craver JM, Jones EL, et al. Surgical managenent of acute myocardial ischemia following percutaneous transhminal coronary angioplasty, Role of the intra-aortic balloon pump. J Thorac Cardionase Surg 1984; 87.332 339 Hoshino M, Asakura $T$, Yasuura $K$, et al. Bypass balloon pumping; a nexty developed device for mechanical circulatory support in heart failure. Thorac Cardiovasc Surg 2003; $51: 2-7$

7 Murashita T, Eya K, Miyatake $T$, et al. Outcome of the perioperative use of percutaneous cardiopulmonary support for adult cardiac surgery: factors affecting hospital mortality. Artif Organs 2004; 28:189-195

8 Doll N, Kiaii B, Borger M, et al. Five-year results of 219 consecutive patients treated with extracorporeal membrane oxygenation for refractory postoperative cardiogenic shock. Ann Thorac Surg 2004; 77:151-157; discussion 157

9 Lazar HL, Treanot P, Yang XM, et al. Enhanced recovery of ischemic myocardium by combining percutaneous bypass with intraaortic balloon pump support. Ann Thorac Surg 1994; 57:663-667; discussion 667-668

10 Pappas G, Winter SD, Kopriva CJ, et al. Improvement of myocardial and other vital organ functions and metabolism with a simple method of pulsarile flow (IABP) during clinical cardiopulmonary bypass. Surgery 1975; 77:34-44

11 Bavaria JE, Furukawa $S$, Kreiner $G$, et al. Effect of circulatory assist devices on stunned myocardium. Ann Thorac Surg 1990; 49:123-128

12 Nanas JN, Poyiadjis A, Charitos $C$, et al. Additional salutary hemodynamic effects of the combined use of the paraortic counterpulsation device and intraaortic balloon pump versus a pataaortic counterpulsation device alone. ASAIO Trans 1990; 36:M505-509

13 Sasaki E, Nakatani T, Taenaka $Y$, et al. A composite driving system for LVAS and IABP: practical and effective driving and weaning. ASAIO Trans 1990; 36:M509. 512

14 Baan J, van der Velde ET, de Bruin $\mathrm{HG}$, et al. Conthuous measurement of left ventricular volume in animals and humans by conductance catheter. Circulation $1984 ; 70: 812-823$

15 Amirhamzeh MM, Dean DA, Jia CX, et al. Validation of right and left ventriculat conductance and echocardiography for cardiac function studies. Ann Thorac Surg 1996; 62:1104-1109

16 Philips $\mathrm{PA}$, Btegman D. Intraoperative application of intranortic balloon counterpulsation determined by clinical monitoring of the endocardial wability ratio. Ann Thorac Surg 1977; 23:45-51

17 Sarnoff SJ, Braunwald E, Welch GH, Jr, et al. Hernodynamic determinants of oxygen consumption of the heart with special reference to the tension-time index. Am J Physiol 1958; 192:148-156 
18 Goldman $\mathbb{B S}_{s}$ Gunstensen J, Gibert BW, et al. Increasing operability and survival with intravortic ballon pump assist. Can J Surg 1976; 19:69-75,78

19 Buckberg GD, Fixler DE, Archie JP, et al. Experimental subendocardial ischemia in dogs with normal coronary arteries. Circ Res 1972; 30:67-81

20 Takaoka $H$, Takeuchi $M$, Odake $M$, et al Comparison of hemodynamic determinants for myocardial oxygen consumption under different contractile states in human ventricle. Circulation 1993; 87:59-69

Suga $H$, Yasumura $Y$, Nozawa $T$, et al. Prospective prediction of $\mathrm{O} 2$ consumption from pressure-volume area in dog hearts. Am J Physiol 1987; 252:H1258-1264

22 Suga $H_{3}$, Hayashi $T$, Shirahata $M$. Ventricular systolic pressure-volume area as predictor of cardiac oxygen consumption. Am J Physiol 1981; 240:H39-44

23 Takaoka $H$, Takeuchi $M$, Odake $M$, et al. Assessment of myocardial oxygen consumption (Vo2) and systolic pressure-volume area (PVA) in human hearts. Eur Heart J 1992; 13 Suppl E:85-90

24 Westerhof N. Cardiac work and efficiency. Cardiovasc Res $2000 ; 48: 4-7$

25 Baller $\mathrm{D}$, Jonas W, Sigmund-Duchanova $\mathrm{H}$, et al. Examination of the validity of the DPTI as an estimate of myocardial oxygen supply with special reference to the DPTI/TTI ratio. Basic Res Cardiol 1978; 73:595-607

26 Buckberg GD, Olinger $G N$, Mulder DG, et al. Depressed postoperative cardiac performance. Prevention by adequate myocardial protection during Caudiopulmonary bypass. J' Thorac Cardiovasc Surg 1975; 70:974-994

27 Buckberg GD, Towers B, Paglia DE, et al. Subendocardial ischemia after cardiopulmonary bypass. J Thorac Cardiovasc Surg 1972; 64:669-684

28 Hughes DA, Igo SR, Daly BD, et al. Effects of an abdominal left ventricular assist device on myocardial oxygen supply/demand ratios in normally perfused and ischemic bovine myocardium. Ann Thorac Surg 1975; :19:301.-308

29 Gibbs CL. Cardiac energetics, Physiol Rev 1978; 58:174-254

30 Laschinger JC, Grossi EA, Cunningham JN, Jr, et al. Adjunctive left ventricular unloading during myocardial reperfusion plays a major role in minimizing myocardial infarct size. J Thorac Cardiovasc Surg 1985; 90:80-85

31 Smalling RW, Cassicty DB, Barrett $R$, et al. Improved regional myocardial blood flow, left ventriculat unloading, and infarct salvage using an axilal-flow, transvalvular left ventricular assist device. A comparison with intra-aortic balloon counterpulsation and reperfusion alone in a canine infarction model. Circulation $1992 ; 85: 1152-1159$

32 Soloff LA. Attophy of myocardium and its myocytes by left ventricular assist device. Circulation 1999; 100:1012

33 Young JB. Healling the heart with ventricular assist device therapy: mechanisms of cardiac recovery. Ann Thorac Surg 2001; 71:\$210-219 
34 Kinoshita $M$, Takano $H$, Takaichi $S$, at al. Infuence of prolonged ventricular assistance on myocardial histopathology in intact heart. Ann Thorac Surg 1996: 61:640-645

35 Kinoshita M, Takano H, Taenatha $Y$, et al. Cardiac disuse atrophy duning LVAD pumping. ASAIO Trans $1988 ; 34: 208-212$

36 Hon $J \mathrm{~K}$, Yacoub MH. Bridge to recovery with the use of left ventricular assist device and clenbuterol. Ann Thorac Surg 2003; 75:S36 -41.

37 Maybaum $S$, Epstein S, Beniaminovitz $A$, et al. Partial loading of the left ventricle during mechanical assist device support is associated with improved myocardial function, blood flow and metabolism and increased exercise capacity. J Heart Lung Transplant 2002; 21:446-454

38 Battlett RH, Roloff DW, Custer JR, et al. Extracotporeal life support: the University of Michigan experience. Jama 2000; 283:904-908

39. Doll $N$, Fabricius $A$, Borger $M A$, et al. Tempotary extracorporeal membrane oxygenation in patients with refractory postopentive cardiogenic shock- a single center experience. J Card Surg 2003; 18:512-518

40 Yasu T, Murata $S$, Katsuki T, et al. Acutely severe myocarditis successfully treated by percutaneous cardiopulmonary support applied by a newly developed heparinbinding oxygenator and circuits. Jpn Circ J 1997; 61:1037-1042 
82| Chapter 4 


\section{Chapter 5}

\section{Physiologic insensitive left ventricular assist predisposes to right-sided circulatory failure: A pilot simulation and validation study}

\section{Published as:}

Reesink K, Dekker A, van der Nagel T, Blom H, Soemers C, Geskes G, Maessen J, van der Veen E. Physiologic-insensitive left ventricular assist predisposes right-sided circulatory failure: a pilot simulation and validation study. Artif Organs 2004; 28:933-939 


\begin{abstract}
Right-sided circulatory failure (RSCF) is a serious complication in 15-30\% of patients receiving a left ventricular assist device (LVAD). We hypothesize that left ventricular support which lacks physiologic properties predisposes to RSCF. An integral computer simulation and experimental validation protocol was performed. The results suggest that with conventional insensitive left ventricular support right-sided circulatory function is compromised, which may form a substrate for the onset or progress of RSCF. Feedback control of the LVAD could provide a means to counter this problem. We propose a LVAD control concept which aims to preserve right-sided circulatory function, while supporting peripheral perfusion.
\end{abstract}

\title{
INTRODUCTION
}

In the past years mechanical circulatory support systems have proved to be clinically viable and successful in certain cases. However, the physiological implications of supporting the circulation with an artificial heart are not entirely understood. There is still a discrepancy in the number of patients successfully assisted clinically and the actual survival rates ${ }^{1,2}$. Nonphysiological properties and limited adaptive qualities of artificial hearts are believed to be key contributors to unsuccessful outcome and associated complications 3.4 .5 . Among the patients that suffer from a substantially compromised circulatory function, unilateral left ventricular failure is the most common indication. A left ventricular assist device (LVAD) may be instituted either as a bridge to recovery or as a bridge to alternative therapies. However, right-sided circulatory failure (RSCF) occurs in 15-30\% of the patients who undergo left ventricular assist therapy $1,6,7$. Currently, two main causes have been suggested and/or identified: Existing RSCF (e.g. right ventricular infarction, pulmonary hypertension), and reduced RV contractility due to leftward shift (anatomical interaction) of the interventriculat septum ${ }^{1,8}$. We hypothesize that functional mismatches between the LVAD and the native circulation (hemodynamic interaction) constitute a third, important factor in the development of RSCF. The 
concept is that the LVAD may push the right heart and lungs towards a region of operation boarding on their capacity limits, thereby creating a substrate for failure or aggravating existing right-sided circulatory fatlure.

The approach of this study was to develop a hemodynamic computer model to study the functional interactions between an LVAD and the right heart and pulmonary circulation. As a pilot study, a simple first order model was developed and validated by a single experimental procedure.

\section{METHODS}

A computer model of the human circulation modified by a continuous flow LVAD was developed using literature data 9,10. An integral computer modeling and experimental validation protocol was conducted to obtain comparable conditions in both arms.

\section{Hemodynamic model}

The computer model is designed to represent a worst case condition with the following features: a) Healthy right heart and lungs, b) no native LV function/output, c) non-pulsatile/continuous flow LV bypass, and d) a non-responsive, constricted systemic vasculat bed. Figure 5.1 shows a block diagram of the model which consists of four sections, each with specific characteristics. From the lower-left, clockwise:

The entire right heart subsection is designed to expose the pump function indicated in Figure 5.1 by the diagram presenting cardiac output (CO) versus right atrial pressure (RAP) ${ }^{10}$. Up to a certain point, right ventricular output is proportional to right atrial pressure (Starling mechanism). Beyond this point output is limited by the ventricle's finite contractility. The position of the curve largely depends on afterload (Fig. 5.1: CO-RAP diagram: Dotted line in case of high afterload), as ejection is entirely valve (i.e. gradient) dependent. In the model maximum right ventricular pressure development is limited to $35 \mathrm{mmHg}$ and the nominal output is $5 \mathrm{l} / \mathrm{min}$ (dot on the curve). 
The pulmonary vascular bed is modeled using the 'waterfall concept' (Fig. 5.1: (PAP-LAP)-CO curve): The flow through the lungs may vary within a wide range, but the pressure gradient across the lungs remains rather constant ${ }^{10,11,12}$. With extreme hypo- or hyperperfusion or lung disease this relationship may alter drastically. The nominal gradient (PAP-LAP) of the lung model is $10 \mathrm{mmHg}$ at $5 \mathrm{l} / \mathrm{min}$ of cardiac output (dot on curve). In the lung outflow region (dotted bold line) the non-linear behavior of pulmonary venous/pump inflow collapse is built in: Whenever left atrial pressure drops below the intrapleural pressure, the pulmonary veins and left atrium collapse, which results in a high resistance to flow (pulmonary congestion).

The selected LVAD model is of the non-pulsatile/continuous flow type which represents the majority of the current rotary blood pumps ${ }^{13}$. Within an acceptable range of circulatory filling volumes, this type can be considered. a flow source that is relatively insensitive to changes in preload and afterload. The pump characteristic in Figure 5.1 shows that pump output (i.e. $\mathrm{CO}$ ) is fairly constant over the (AoP-IAP) range: The LVAD delivers its output almost regardless of the pressure gradient across it. The LVAD is set to a fixed, nominal output of $5 \mathrm{l} / \mathrm{min}$. Fixed output implies non-responsiveness to physiology (uncontrolled LVAD). Despite numerous research and development efforts to build feedback control into LVAD systems ${ }^{3,14}$, in current clinical practice this setting is often standard ${ }^{15}$.

Because the interactions of the periphery with an artificial left heart are still unclear ${ }^{5,13,16}$, modeling of the peripheral vascular bed is kept straightforward. At a nominal cardiac output of $5 \mathrm{l} / \mathrm{min}$ mean aortic pressure is set to around $90 \mathrm{mmHg}$.

The overall hemodynamic characteristics are based on a healthy, male adult weighing $80 \mathrm{~kg}$, with a nominal resting cardiac output of $51 / \mathrm{min}$; arterial blood pressures of $120 / 80$ and a fixed circulatory volume of 5 liters. In steady state conditions cardiac output (venous return) is continuous throughout the cycle (Fig. 5.1). Heart rate within the model is fixed at 80 bpm. 


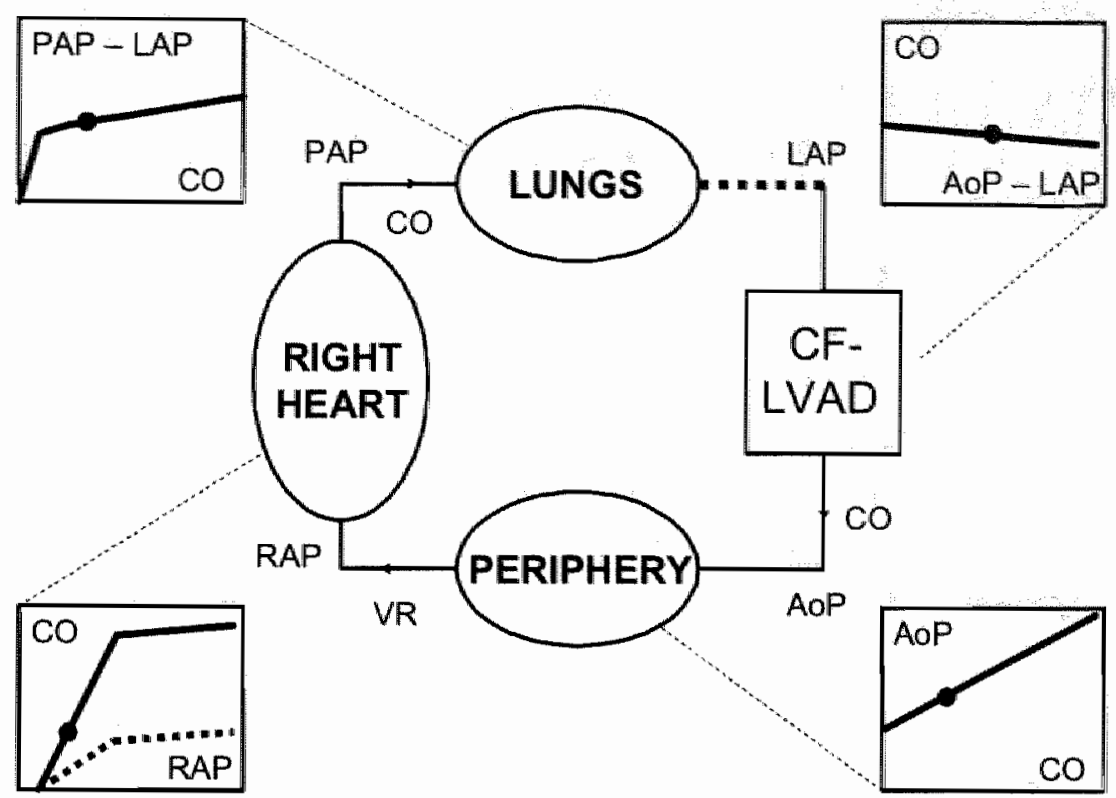

Figure 5.1 Network model of the assisted circulation. PAP: pulmonary artery pressure, LAP: left atrial pressure, AoP: aortic pressure, RAP: right atrial pressure, CO: cardiac output, VR: venous return, CF-LVAD: continuous flow left ventricular assist device. Dots on each curve indicate nominal working conditions.

\section{Experimental model}

A $109 \mathrm{~kg}$ calf was premedicated with attopine $(0.05 \mathrm{mg} / \mathrm{kg} \mathrm{s.c.)}$. Anesthesia was induced with sodium thiopental (i.v.-bolus $20 \mathrm{mg} / \mathrm{kg}$ ) and maintained with a $1: 2$ mixture of $\mathrm{O}_{2}: \mathrm{N}_{2} \mathrm{O}$ and halothane $(1.5 \%)$. After administration of analgesic buprenorfine (i.v.-bolus $0.01 \mathrm{mg} / \mathrm{kg}$ ) and muscle relaxant suxamethonium (i.v.-bolus $0.1 \mathrm{mg} / \mathrm{kg}$ ), a left thoracotomy was performed during which PEEP was set to $5 \mathrm{cmH}_{2} \mathrm{O}$. Coagulation was controlled through administration of heparin (i.v.-bolus $200 \mathrm{IU} / \mathrm{kg}$ ) and monitored by activated clotting time (ACT) measurements. ACT was kept above 400 seconds during the experiment. Monitoring included ECG, blood pressure, oxygen saturation and capnography. The animal was sactificed through a pentobarbital overdose (i.v.-bolus $200 \mathrm{mg} / \mathrm{kg}$ ). The animal received humane care in compliance with the "Guide for the Care and Use of Laboratory 
Animals" (NIH publication 86-23, 1985 revision; National Institutes of Health, USA, www.nih.gow).

Full LV bypass was established through institution of an extracorporeal circuit featuring left atrial and proximal aortic cannulation, an inflow teservoir and a centrifugal pump (BIO-PUMP BP-80, Medtronic Bio-Medicus, Inc., Eden Prairie, MN, USA). LV form and function were left intact.

Diagnostic instrumentation included a Swan-Ganz thermodilution catheter, aortic- and pulmonary artery pressure catheters (Sentron, Rhoden, The Netherlands), a RV conductance catheter (CD Leycom, Zoetermeer, The Netherlands) ${ }^{17,18}$, an ultrasonic pulmonary artery flow probe (Transonic Systems Inc., Ithaca, NY, USA), pump inflow cannula ( left atrial pressure: LAP) and right atrial (RAP) standard pressure transducers, and a pump outflow probe. All data was acquired at a sampling rate of 250 $\mathrm{Hz}$ and stored on hard disk.

\section{Interventions}

In both the simulation and experimental protocols two interventions were performed: Sudden elevation of right ventricular (RV) afterload, and increase in circulatory volume. The computer model was exercised by changing the model variables and parameters to simulate the interventions. Experimentally, RV afterload was changed by transiently constricting the pulmonary artery through banding. Blood volume was increased by infusion of 1 liter of blood from the extracorporeal reservoir. Maximum LVAD flow was limited to the full bypass condition (no LV output).

\section{Analysis}

Data analysis included all measured hemodynamic variables (HR, pressures, flows and right ventricular volume) and the following right ventricular indices: SV: Stroke volume, EF: (right ventricular) Ejection fraction, SW: Stroke work (= SV.(PRV peak $-\mathrm{EDP})$ ), TPG: Trans pulmonary gradient (= 
PAP-LAP) and PVR: Pulmonary vascular resistance (- TPG/CO). The right ventricular output derived from the conductance data was calibrated using the pulmonary artery flow.

Table 5.1 Comparison of nominal hemodynamic profiles

\begin{tabular}{llccc}
\hline & & Simulation & Experiment & $\begin{array}{c}\text { scale } \\
\text { factor }\end{array}$ \\
\hline HR & bpm & 80 & 100 & 1.3 \\
PAF & $1 / \mathrm{min}$ & 5.1 & 7.5 & 1.5 \\
POF & $1 / \mathrm{min}$ & 5.1 & 7.6 & 1.5 \\
EDV & $\mathrm{ml}$ & 123 & 188 & 1.5 \\
ESV & $\mathrm{ml}$ & 59 & 113 & 1.9 \\
PRV ${ }_{\text {peak }}$ & $\mathrm{mmHg}$ & 22 & 4.9 & 2.2 \\
PAP & $\mathrm{mmHg}$ & 13 & 18 & 1.4 \\
LAP & $\mathrm{mmHg}$ & 0.4 & $-20 *$ & $*$ \\
AoP & $\mathrm{mmHg}$ & 88 & 99 & 1.1 \\
RAP & $\mathrm{mmHH}$ & 3.5 & 2.3 & 0.7 \\
\hline SV & $\mathrm{ml}$ & 64 & 75 & 1.2 \\
EF & $\%$ & 52 & 40 & 0.8 \\
SW & $\mathrm{J} / \mathrm{beat}$ & 1.2 & 3.5 & 2.9 \\
PVR & $\mathrm{mmHg} \min / 1$ & 2.5 & 5.1 & 2.0 \\
TPG & $\mathrm{mmHg}$ & 13 & 38 & 2.9 \\
\hline
\end{tabular}

Values are mean values. Scale factor $=$ experimental value/simulated value. All cardiac indices are fight ventricular (RV), unless otherwise indicated. HR: heart rate, PAF: pulmonary artery flow, POF: left ventricular assist pump outflow, EDV: end diastolic volume, ESV: end systolic volume, PRV preak: peak RV pressure, PAP: pulmonary artery pressure, LAP: left atrial ptessure, AoP: aortic pressure, RAP: right atrial pressure, SV: stroke volume, EF: RV ejection fraction, SW: stroke work, PVR: palmonary vascular resistance, TPG: trans pulmonary gradient. *Scale factor not appropriate to state. Note that LAP was measured at the inflow cannula of the LVAD. 
901 Chapters 5

\section{RESULTS}

Nominal physiologic state

In both models, the stable condition of the circulation supported by the LVAD in full LV bypass mode is considered baseline. Table 5.1 compares the hemodynamic profiles of the simulation and experimental models.

\section{Pulmonary artery constriction}

Figure 5.2 shows the beat-to-beat tracings of a pulmonary artery constriction. With the progression of the constriction a mismatch in pump outflow (POF) and pulmonary artery flow (PAF) becomes evident. Right ventricular volumes increase due to a loss of right ventricular output and a

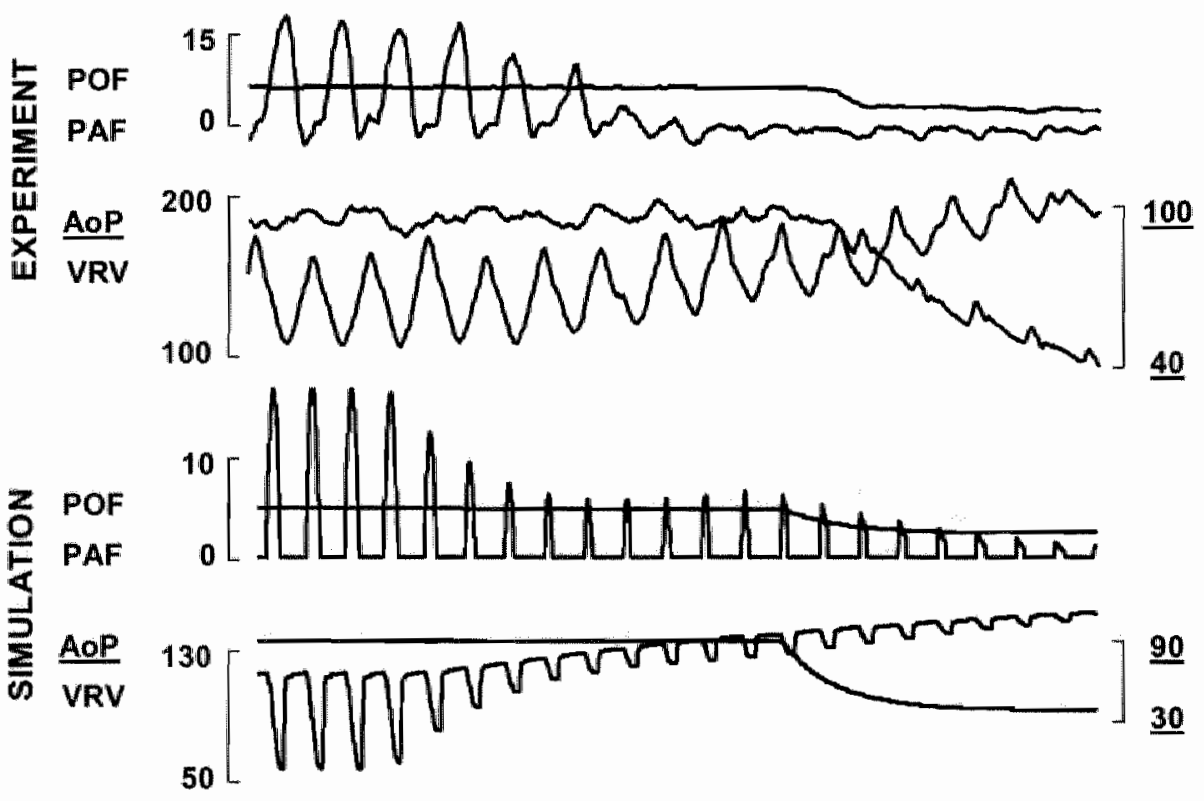

Figure 5.2 Simulated and experimental hemodynamic trends recorded during pulmonary artery constriction. POF: pump outflow, PAF: pulmonary artery flow, AoP: aortic pressure, VRV: tight ventricular wolume. The aotric pressure scales ate given on the right side. 
EXPERIMENT

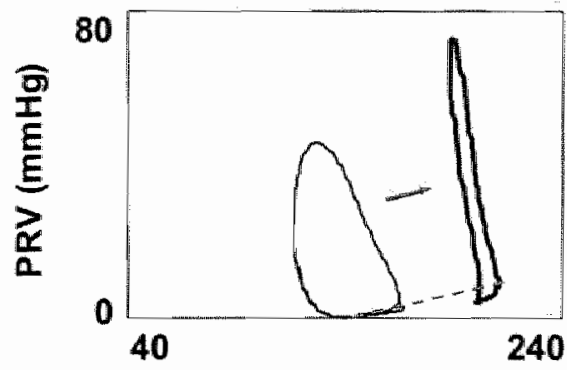

SIMULATION

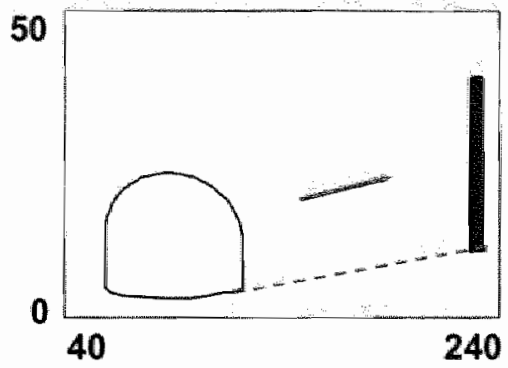

VRV (ml)

Figure 5.3 Right ventricular work before (thitn trace) and during (thick trace) pulmonary artery constriction. PRV: right ventriculas pressure, VRV: right ventricular volume.

relatively stable venous retum (POF). Suction at the LVAD inflow cannula as a tesult of severe congestion is noted from the drop in POF and AoP. The bypass pump cannot maintain its output because it lacks preload. In the experiment, LAP dropped from -20 down to $-200 \mathrm{mmHg}$ (the instrumentation's range limit), which supports the occurrence of severe suction and pump inlet collapse.

The right ventricular pressure-volume relationships in the pulmonary artery occlusion case are given in Figure 5.3. A pronounced decrease in stroke volume is seen in both cases, though it is accompanied by a considerable increase in right ventricular filling. The end-diastolic pressure and volume elevations were more pronounced in the simulation.

\section{Increased circulatory volume}

The hemodynamic changes with the circulatory filling intervention are summarized in Table 5.2. The experimental baseline data were obtained just. before infusion of 1 -liter of blood from the extracorporeal circuit's venous reservoir.

In both models right ventricular peak pressure, pulmonary pressures (PAP and LAP), and right ventriculat work (SW) increased comparably. In 
both models the thans pulmonary gradient (TPG) remained fairly constant. Only in the experimental model pulmonary vascular resistance changed (PVR -36\%). In the experimental model cardiac output and aortic pressure increased $(+42 \%$ and $+19 \%$ respectively), whereas during the computer simulation peripheral perfusion hardly changed at all. In vivo, post intervention cardiac output (PAF) was greater than the assist pump's output (Table 5.2). Apparently, the LV adds $2.1 \mathrm{l} / \mathrm{min}$ to the bypass flow $(6.5$ $\mathrm{l} / \mathrm{min})$, which yields a cardiac output of $8.6 \mathrm{I} / \mathrm{min}$.

Table 5.2 Hemodynamic changes after increase of circulatory volume

\begin{tabular}{|c|c|c|c|c|c|c|c|}
\hline & & \multicolumn{2}{|c|}{ Simulation } & \multicolumn{4}{|c|}{ Experiment } \\
\hline & & Baseline & $\begin{array}{l}\text { Increased } \\
\text { Volume }\end{array}$ & $\begin{array}{c}\text { Relative } \\
(\%)\end{array}$ & Baseline & $\begin{array}{l}\text { Increased } \\
\text { Volume }\end{array}$ & $\begin{array}{c}\text { Relative } \\
(\%)\end{array}$ \\
\hline $\mathrm{HR}$ & bpm & 80 & 80 & 0 & 101 & 109 & +8 \\
\hline PAF & $1 / \mathrm{milin}$ & 5.12 & 5.14 & 0 & 6.01 & 8.55 & +42 \\
\hline $\mathrm{POF}^{2}$ & $1 / \mathrm{min}$ & 5.12 & 5.14 & 0 & 6.68 & 6.50 & 0 \\
\hline PRV & $\mathrm{mm} / \mathrm{lg}$ & 22 & 24 & +9 & 49 & 57 & +16 \\
\hline $\mathrm{PAP}$ & $\mathrm{mmHg}$ & 13 & 15 & +15 & 17 & 21 & +24 \\
\hline I.AP & $\mathrm{mm} H \mathrm{~g}$ & 0.4 & 2.4 & $*$ & -18 & -11 & * \\
\hline Aop & $\mathrm{mmHg}$ & 88 & 89 & 0 & 73 & 87 & +19 \\
\hline RAP & $\mathrm{mmHg}$ & 3.5 & 3.8 & $*$ & 0.1 & 5.4 & $*$ \\
\hline SV & $\mathrm{ml}$ & 64 & 65 & 0 & 59 & 78 & +32 \\
\hline SW & $\mathrm{J} /$ beat & 1.19 & 1.33 & +12 & 2.68 & 3.64 & +36 \\
\hline PVR & $\begin{array}{l}\operatorname{mn} H g " \\
\min / 1\end{array}$ & 2.5 & 2.5 & 0 & 5.8 & 3.8 & -36 \\
\hline $\mathrm{TPG}$ & $\mathrm{mmHg}$ & 13 & 13 & 0 & 35 & 32 & -9 \\
\hline
\end{tabular}

Values ate menn values. All cardiac indices are right ventricular (RV). HR: heart rate, PAF: pulmonary artery flow, POF: left ventricular assist pump outflow, PRV peak: peak RV pressure, PAP: pulmonary artery pressure, IAP: left atrial pressure, AoP: aortic pressure, RAP: right atrial pressure, SV: stroke volume, SW: stroke work, PVR: pulmonary vascular teststance, TPG: trans pulnonary gradient. * Relative change values not suitable to state. 
If we analyze the differences between the model responses to the filling intervention, we can conclude the following. During simulation citculatory filling did not increase peripheral perfusion, but required an increase in tight ventricular work, because of increased pulmonary loading (Table 5.2). In the experimental model circulatory filling increased the load on the right side, but improved peripheral perfusion concomitantly. This increase in cardiac output was the result of additional native left ventricular output invoked by increased left heart preload (LAP went up $+7 \mathrm{mmHg}$ ). We now define a measure for the cost-to-effort relationship based on right ventricular work and peripheral perfusion: (SW.HR)/CO, with units: [J/beat] [ [beats/min] $/[1 / \mathrm{min}]=[1 /]$. This ratio defines the energy the right ventricle needs to transport one liter of blood. At baseline (SW.HR)/CO was: $19 \mathrm{~J} / 1$ (simulation) and $45 \mathrm{~J} / \mathrm{l}$ (experiment). The post intervention values are $21 \mathrm{~J} / \mathrm{l}$ and $47 \mathrm{~J} / 1$, respectively. Figure 5.4 shows (SW/HR)/CO as a function of peripheral perfusion enhancement. The responses are normalized to enable comparison.

\section{EXPERIMENT}

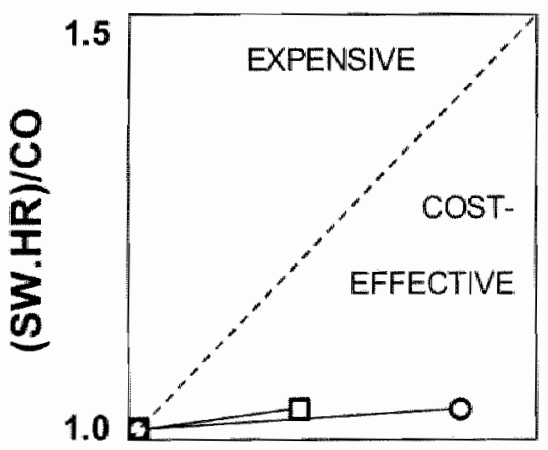

1.0

1.51 .0

SIMULATION

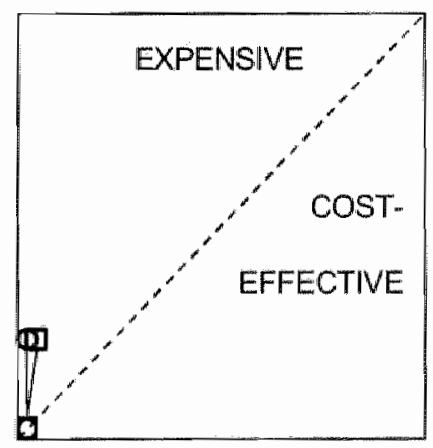

1.5

$$
\square=\mathrm{CO} \quad \mathrm{O}=\mathrm{AOP}
$$

Figure 5.4 Nomalized right ventricular transpot expense index (SW-HR)/CO, weighted by the augmentation in peripheral perfusion (as a result of volume loading). The dashed diagonal indicates a one-tomone proportional cost/benefit relation. 
$94 /$ Chapter 5

\section{DISCUSSION}

\section{Model validation}

The nominal hemodynamic profiles of both models showed good agreement (Table 5.1). The main differences could be explained through the influence of factors like age and body mass. Pulmonary artery constriction indicated good correlation between model dynamics (Figs. 5.2 and 5.3). These results indicate that the computer model has the desired structural and functional properties to study congestive failure of the pulmonary circulation and interactions with the LVAD and systemic circulation. Not yet included in this pilot study was a model of right-sided circulatory failure being either right ventricle or lung based.

Increasing circulatory volume identified major differences in the model responses. In the experimental model, filling raised left heart preload and addressed the native left ventricle to increase forward cardiac output (Table 5.2). Cardiac output did not increase in the simulation as the fixed output LVAD cannot respond to preload elevation. In vivo, the 'fully emptied LV'/LVAD tandem actually did act as a preload sensitive assist device, with the left ventricle being responsible for the adaptation. Because the study was designed as a pilot, full development of a true dysfunctional LV model was not achieved.

\section{Right-sided circulatory overload}

The primary aim of this study was to identify whether physiologic insensitive LV support compromises right-sided circulatory function. From Figure 5.2 it is clear that pulmonary congestion caused by severe LVAD suction not only compromises cardiac output, but also increases loading of the right heart. Consequently, right heart pump function will deteriorate (Fig. 5.1: dotted CO-RAP curve) and overdistension of the right ventricle and atrium will ensue (Figs. 5.2 and 5.3). Volume overload of the atria is associated with atrial fibrillation ${ }^{19}$. Furthermore, the decrease in aortic pressure and cotonary perfusion may lead to right ventricular hypoxia. The 
pulmonary artery constriction was rather severe and acute, but it illustrates one of the underlying mechanisms present in gradual progression of RSCF. Had the LVAD been sensitized to preload, pulmonary circulatory overload may have not developed.

Circulatory filling stressed the importance of preload sensitivity in the regulation of cardiac output and circulatory pressures (Table 5.2). During the simulated intervention peripheral perfusion did not change. In vivo however, the left-sided pump (LV-LVAD tandem) did prove sensitive to preload elevation and increased cardiac output consequently. This physiological response restores the volume balance that is crucial to maintain good circulatory function. Hemodynamic balance between the right and left side of the circulation is not only maintained by Starling's law of the heart, but through adaptations in the pulmonary and systemic vascular beds as well. Table 5.2 shows a considerable decrease in PVR $(-36 \%)$ in vivo in response to increased pulmonary loading. By regulating pulmonary pressures over a broad range of cardiac outputs, the lungs maintain proper gas exchange. In the simulation model pulmonary adaptation was not addressed, because cardiac output was not increased and consequently disproportionate loading of the tight side developed (SW $+12 \%, \mathrm{PRV}_{\text {peak }}+9 \%, \mathrm{PAP}+15 \%$, and $\mathrm{LAP}+2 \mathrm{mmHg}$ ).

Analysis of the expenses with increased circulatory loading based on right ventricular work again stresses the need for preload sensitive $\mathrm{LV}$ support (Fig. 5.4). In the non-adaptive case (simulation) the right ventricle must perform more work per unit of cardiac output, while no perfusion benefit is seen. In the experimental case right-sided circulatory function is preserved, because the 'hybrid LVAD' proportionately responds to elevated pulmonary venous return. Though no actual right heart or pulmonary failure was created or modeled, the differences in dynamics exposed in Figure 5.4 are exemplary. Naturally, the significance of this effect becomes ever greater in pathophysiological situations. Existing right heart failure and pulmonary congestion limit the acceptable margin for the LVAD to operate in: With failure, the 'cost-effective' range will be diminished and consequently LVAD adjustment will be more critical. 


\section{Control considerations}

LVAD support can be indicated for a wide range of diagnoses. Anticipated duration and prognosis determine the requirements on device selection, installation and maintenance. In patients with recoverable left ventricular function the main goal would be to facilitate recovery, while preserving the remainder of circulatory functions. A LVAD feedback control that combines these two objectives may reduce the incidence of RSCF or promote the recovery process. In patients receiving long term left ventricular support (e.g. as a bridge to transplantation) the integral approach to LV assist would be even more critical, as mismatching would occur more often or for longer periods of time and could then cumulatively induce failure. To prevent these events of circulatory imbalance, LVAD control should necessarily be focused on right-sided circulatory function. Firstly, control should stabilize steady state hemodynamics, i.e. the algorithm must cope with variations in circulatory dynamics and keep the LVAD and circulation operating within a 'feasible range'. This also implies that measurements include functional indices from both the left and right side. Secondly, periodic interventions should be performed to evaluate the status of the LVAD's and pulmonary circulation's dynamic function. A suitable intervention (under close monitoring) could be a sudden drop in pump speed, followed by a quick return to normal after 30-60 seconds. The observed circulatory response then provides information on whether the steady state adjustment of the LVAD is still suitable or that it needs to be changed. These interventions should play an important role in the final stages of weaning as well.

\section{Conclusion}

The results of this study suggest that with conventional left ventricular assist therapy (fixed output mode LVAD), in the face of circulatory changes, the right heart and the lungs may be forced into less advantageous modes of operation. We propose a LVAD control concept which aims to preserve 
trght-sided circulatory function at all times, while supporting peripheral perfusion.

\section{References}

1 Chen JM, Rose EA. Management of periooperative right-sided circulatory fallure. In: Goldstein DJ, Oz MC, eds. Cardiac assist devices. First edition. Amonk, NY: Futura Publishing, 2000: 83-101

2 Kumpati GS, McCarthy PM, Hoercher $\mathrm{K}$. Left ventricular assist device bridge to recovery: a review of the current status. The Annals of Thoracic Surgery 2001; $71(1): \$ 103-8$

3 Trinkl J, Havlik P, Mesana T . Control of a rotary pulsatile cardiac assist pump driven by an electric motor without a pressure sensor to avoid collapse of the pump inlet. Asaio J 1993; 39:M237-41

4 Dekker AL, Geskes GG, Cramers AA, Dassen WR, Maessen JG, Prenger $\mathrm{KB}$, Van der Veen FH. Right ventricular support for off-pump coronary artery bypass grafting studied with bi-ventricular pressure-volume loops in sheep. European Journal of Cardio-thoracic Sungery 2001; 19:179-84.

5 Kormos RL, Frazier OH, Griffith BP, Magovetn JA, Noon G P, Wolner E. Discussion of implantable nonpulsatile devices. The Annals of Thoracic Surgery 2001; 71(1):S144-6

6 Farrar D, Compton P, Hershon J, Hill JD. Right ventricular function in an operating room model of mechanical left ventricular assistance and its effects in patients with depressed left ventricular function. Circulation 1985; 72(6):1279-85.

7 Mandarino WA, Kormos RL, Kawai A, Gasior TA, Pinsky MR, Griffith BP. Dynamic biventricular response to alterations in preload in patients undergoing left ventricular device implantation. Asaio J 1994; 40:M295-8

8 Fartar DJ. Physiology of ventriculat interactions during wentriculat assistance. In: Goldstein DJ, Oz MC, eds. Cardiac assist devices. First edition. Armonk, NY: Futura Publishing, 2000: 15-26

9 Nichols WW, Pepine CJ. Ventricular/vascular interaction in health and heart failure. Comprehensive Therapy 1992; 18(7):12-9

10 Guyton AC, Hall JE, eds. Textbook of Medical Physiology. 9 th edition. Philadelphia: W.B. Saunders Company, 1996

11 Naeje R, Lejeune P, Leeman M. Pulmonary arterial pressure-flow plots in dogs: effects of isoflurane and nitroprusside. J Appl Physiol 1987; 63(3):969-77

12 Jeeman $M$. The pulmonary circulation in acute lung injury: a review of some recent advances. Intensive Care Med 1991; 17(5):254-60 


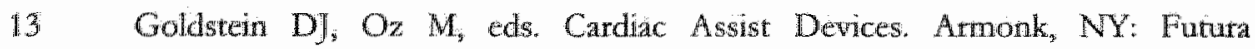
Publishing Company, 2000

14 McInnis BC, Cuo ZW, La PC, wang JC. Adaptive control of left pentricular bypass assist devices. IEEE Transactions on Automatic Control 1985; AC30:322-9

15 Muller $\mathrm{E}$, Munch $\mathrm{K}$. Servoregulation of centrifugal pumps. A new technical approach to improve patient safety during longterm extracorporeal life support. Asaio J 1996; 42(4):282-7

16 Konishi H, Antaki JF, Boston JR, Kerrigan JP, Mandarino WA, Yarnazaki K, Oe M, Lirwak P, Borovetz $H S$, Butler KC, Kormos RL. Dynamic systemic vascular resistance in a sheep supported with a Nimbus AxiPump. Asaio J 1994; 40:M299. 302

17 Batan J, Van der Velde ET, De Bruin HG, Smeenk GJ, Koops J, Van Dijk AD, Temmerman D, Senden J, Buis B. Continuous measurement of left ventricular volume in animals and humans by conductance catheter. Circulation 1984; $70: 812-23$

18 Schreuder J, Van der Veen FH, Van der Velde ET, Delahaye F, Alfieri O, Jegaden $O$, Lorusso $R$, Jansen JRC, Hoeksel SAAP, Finet $G$, Volterrani $M$, Kaulbach $H G$, Baan $J$, Wellens HJJ. Left ventricular pressure-wohume relationships before and after cardiomyoplasty in patients with heart failure. Circulation 1997; 96(9):2978-86

19 Schotten $U$, Neuberger $H \mathbb{R}$, Allessie MA. The role of atrial dilatation in the domestication of atrial fibrillation. Prog Biophys Mol Biol 2003; 82:151-62 
Intracardiac assist device 199

\section{Chapter 6}

\section{Miniature intracardiac assist device provides more effective cardiac unloading and circulatory support during severe left heart failure than intra-aortic balloon pumping}

\section{Published as:}

Reesink KD, Dekker AL, Van Ommen V, Soemers C, Geskes GG, van der Veen FH, Maessen JG. Miniature intracardiac assist device provides more effective cardiac unloading and circulatory support during severe left heart failure than intraaortic balloon pumping. Chest $2004 ; 126: 896-902$ 


\section{ABSTRACT}

\section{Background}

Miniature intracardiac pump (IMP) assistance may fill the gap between intra-aortic balloon pumping ( $\mathrm{ABP}$ ) and the current, more invasive ventricular assist devices. The objective of this study was to compare the hemodynamic efficacy of an IMP device with IABP.

\section{Methods}

Reversible acute mitral regurgitation (AMR) was induced in 8 calves by stenting the mitral valve using a vena cava filter. Full and partial IMP assist were compared with maximum IABP support in each animal.

\section{Resuits}

In full support mode both assist systems (IMP / IABP; p-values: Pump onoff comparison) increased cardiac output $(+13 \%, \mathrm{p}<0.05 /+3 \%, \mathrm{p}<0.05)$, mean aortic pressure $(+13 \%, \mathrm{p}<0.05 /+8 \%, \mathrm{p}<0.05)$, carotid artery flow $(+29 \%, \mathrm{p}<0.05 /+5 \%, \mathrm{p}=\mathrm{NS})$, and coronary blood flow $(+25 \%, \mathrm{p}=\mathrm{NS} /$ $+34 \%, \mathrm{p}<0.05)$. Left atrial pressure $(-2.4 \mathrm{mmHg}, \mathrm{p}<0.05 /-0.7 \mathrm{mmHg}$, $\mathrm{p}<0.05)$, peak left ventricular pressure $(-13 \%, \mathrm{p}<0.05 /-5 \%, \mathrm{p}<0.05)$, and external left ventricular work $(-29 \%, \mathrm{p}<0.05 /-3 \%, \mathrm{p}<0.05)$ were reduced. Only full JMP support reduced both end-diastolic $(-7 \%, \mathrm{p}<0.05)$ and endsystolic $(-10 \%, p<0.05)$ left ventricular volume. IABP mainly improved coronary perfusion while the IMP proved more capable of genuinely unloading the left ventricle.

\section{Contlusions}

We conclude that during severe acute left ventricular failure, the miniature intracardiac pump is capable of more effective cardiac unloading and circulatory support than intra-aortic balloon pumping. 


\section{INTRODUCTION}

During the past four decades intra-aortic balloon pumping (ABP) has established itself as the standard mechanical cardiac assist option to treat patients in acute cardiogenic shock ${ }^{1.5}$. In search of more effective alternatives, new intravascular devices have been developed and used for cardiac support in postcatdiotomy cardiogenic shock and during coronary artery bypass surgery ${ }^{6-9}$.

The Impella intracardiac microaxial pump (IMP; Impella Cardiotechnik AG, Aachen, Germany) is a new, catheter-based cardiac assist device designed to provide ventricular support in bypass mode. Although the IMP has been introduced clinically recently, the hemodynamic efficacy has not been extensively evaluated yet ${ }^{10-11}$. In the present study, IMP left ventricular support and IABP were evaluated in an animal model of acute mitral regurgitation ${ }^{5}$.

\section{METHODS}

\section{Animal preparation}

Eight calfs (mean $81 \mathrm{~kg}$, range $60-92 \mathrm{~kg}$ ) were premedicated with atropine $(0.05 \mathrm{mg} / \mathrm{kg}$ s.c.). Anesthesia was induced with sodium thiopental (i.v. bolus $20 \mathrm{mg} / \mathrm{kg}$ ) and maintained with a $1: 2$ mixture of $\mathrm{O}_{2} \cdot \mathrm{N}_{2} \mathrm{O}$, and halothane $(1.5 \%)$. After administration of analgesic buprenotfine (i.v. bolus 0.01 $\mathrm{mg} / \mathrm{kg}$ ) and muscle relaxant suxamethonium (i.v. bolus $0.1 \mathrm{mg} / \mathrm{kg}$ ), a left thoracotomy was performed. The anticoagulation regimen was: Heparin (i.v. bolus $200 \mathrm{IU} / \mathrm{kg}$ ); monitoring by activated clotting time (ACT $>400 \mathrm{~s}$ ). Animal sacrifice was by pentobarbital overdose (i.v. bolus $80 \mathrm{mg} / \mathrm{kg}$ ). All animals received humane care in compliance with the "Guide for the Care and Use of Laboratory Animals" (NIH publication 86-23, 1985 revision; National Institutes of Health, USA) and the study was approved by the university's animal ethical committee. 
$102 \mid$ Chupter 6

\section{Cardiac support systems}

The IMP (Impella Cardiotechnik AG, Aachen, Germany) features a $6.4 \mathrm{~mm}$ diameter; $100 \mathrm{~mm}$ long integrated cannula-pump assembly, suitable for placement across the aortic valve. The pump's axial impeller (Archimed screw) continuously recruits blood from the left ventricle via the cannula and drains the blood into the aorta just above the aortic valve. The pump housing contains an integrated solid-state differential pressure sensor that registers the pressure gradient across the pump. The device is powered and controlled by a pump console via a connecting catheter. Placement of the catheter pump is via percutaneous access or intraoperatively via aortic cannulation. The pump is designed for short to mid (maximum 7 days) term support ${ }^{12-13}$.

The IMP was introduced via the left carotid artery and placed across the aortic valve with the inflow cannula in the left ventricle and pump housing and outlet just above the aortic valve. Positioning was facilitated by the pressure gradient reading on the pump console ${ }^{11-43}$. Routine checks with fluoroscopy confirmed proper placement of the pump. Immediately after placement of the IMP the pump rate was set to a minimum level, sufficient to prevent aortic retrograde flow. The IMP console flow measurement was calibrated by the automated console calibration sequence after pump positioning.

A $40 \mathrm{ml}$ intra-aortic balloon catheter (Datascope, Fairfield, NJ) was introduced via the right femoral artery and placed in the high descending aorta. The intra-aortic balloon pump (System 98 XT; Datascope, Fairfield, NJ) was triggered on the ECG.

\section{Instrumentation}

A Swan-Ganz thermodilution catheter (Arrow International, Reading, PA) was advanced into the pulmonary artery via a jugular vein. A conductance carheter, incorporating a pressure sensor (CD Leycom, Zoetermeer, the Netherlands), was positioned in the left ventricle via left femoral arterial access. The conductance catheter was connected to a conductance console 
(Sigma 5/DF, CD Leycom, Zoetermeer, the Netherlands) which was used in dual-frequency mode ${ }^{14}$. Solid-state pressure catheters (CD Leycom, Zoetermeer, the Netherlands) were placed in the abdominal aorta wia the left femoral artery and in the left atrium through a purse-string suture. Blood pressure in the aortic arch was measured wia the intra-aortic balloon catheter's lumen with a standard pressure transducer (Baxter International Inc., Deerfield, IL). Ultrasonic flow probes (Transonic Systems, Ithaca, NY) were placed on the pulmonary artery, the right carotid artery and on a side branch of the left anterior descending coronary artery. All hemodynamic data were acquired and stored using a 16-channel acquisition system (CD Leycom, Zoetermeer, The Netherlands).

To induce acute mitral regurgitation (AMR) an incision was made in the left atrium through which a retrievable steel wire cage (Günther tulip vena cava filter, Cook Europe, Bjaeverskov, Denmark) was placed in the mitral valve ${ }^{5-9}$. The degree of mitral regurgitation was assessed and controlled by real-time left ventricular pressure-volume and left atrial pressure monitoring. The induction of mitral regurgitation was consistently aimed at maximizing the regurgitant orifice.

\section{Conductance calibration}

Parallel conductance was determined by injecting $7.5 \mathrm{ml}$ of $6.5 \%$ hypertonic saline into the pulmonary artery ${ }^{15}$. A $5 \mathrm{ml}$ blood sample was collected in a sampling cuvette for blood resistivity measurement (CD Leycom, Zoetermeer, the Netherlands) ${ }^{16}$. The gain calibration factor was determined by comparing LV conductance derived output (CDO) with the pulmonary artery flow in the healthy animal. The conductance data was analysed with the Circlab 2000 software package (GTX Medical Software, Leiden, the Netherlands).

\section{Measurements and analysis}

Both IABP and the IMP were tested in each animal, in an alternated sequence every consecutive experiment. The intra-aortic balloon pump was 
driven in 1:1 mode and tuned to maximize left ventricular afterload reduction and diastolic aortic pressure augmentation ${ }^{1.3}$. The IMP was run at medium and a full speed (respectively 24,000 (performance level P5) and 33,000 rpm (P9)). Approximately 10 seconds after the creation of mitral regurgitation, assist was initiated and maintained for 30 seconds.

The hemodynamic data are analysed and processed to obtain objective measures for left heart pump function, myocardial working conditions, and peripheral perfusion. At baseline (i.e. during calibration) the conductance derived output ( $\mathrm{CDO}=\mathrm{SV} \cdot \mathrm{HR}$ ) is set equal to pulmonary artery flow $\left(\mathrm{Q}_{\mathrm{PA}}\right)$, and the flow through the aortic valve $\left(\mathrm{Q}_{\mathrm{AV}}\right)$ is considered

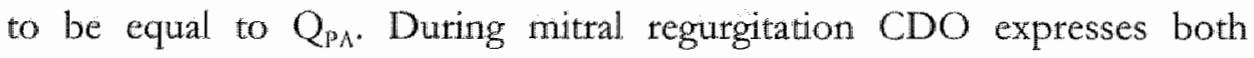
forward $\left(Q_{A V}\right)$ and regurgitant output across the stented mitral valve $\left(Q_{R}\right)$, and thus $\mathrm{CDO}=\mathrm{Q}_{\mathrm{AV}}+\mathrm{Q}_{\mathrm{R}}(\mathrm{l} / \mathrm{min})$. During IMP support part of the forward output is no longer through the aortic valve but via the pump, and $Q_{A V}$ is calculated from: $Q_{P A}-Q_{M M P}$. $Q_{A V}$ is solely due to contraction of the LV. $Q_{\text {IMP }}$ is the flow measured through the IMP. Forward output $\left(Q_{P A}\right)$ is then the result of the combined effects of LV contraction and IMP aspiration.

Pulse pressure (PP) was calculated as the maximum-minimum differential of the ascending aortic pressure. Diastolic coronary flow fraction (DCF) was calculated as the ratio of antegrade diastolic coronary flow over mean coronary flow, times $100 \%$. External left ventricular work (LVW) was calculated as follows: $\left.\left[\mathrm{PLV}_{\text {peak }}-\mathrm{LAP}\right] \cdot\left[\mathrm{Q}_{\mathrm{AV}}+\mathrm{Q}_{\mathrm{R}}\right] \mathrm{O} / \mathrm{s}\right)$. The ratio of cardiac output over left atrial pressure ( $\left.\mathrm{Q}_{\mathrm{P}} / \mathrm{LAP}\right)$ was calculated as an index of left heart purmp function ${ }^{17}$. A measure for the balance in coronary demand and supply was defined by the LVW/coronary flow ratio $(\mathrm{J} / \mathrm{ml}$ ). For each setting the on (AMR plus assist) and off (AMR plus assist idle) conditions were compared by application of the non-parametric Wilcoxon signed-ranks test $(n=8)$. Significance was assumed if $p$-values were lower than 0.05 . 


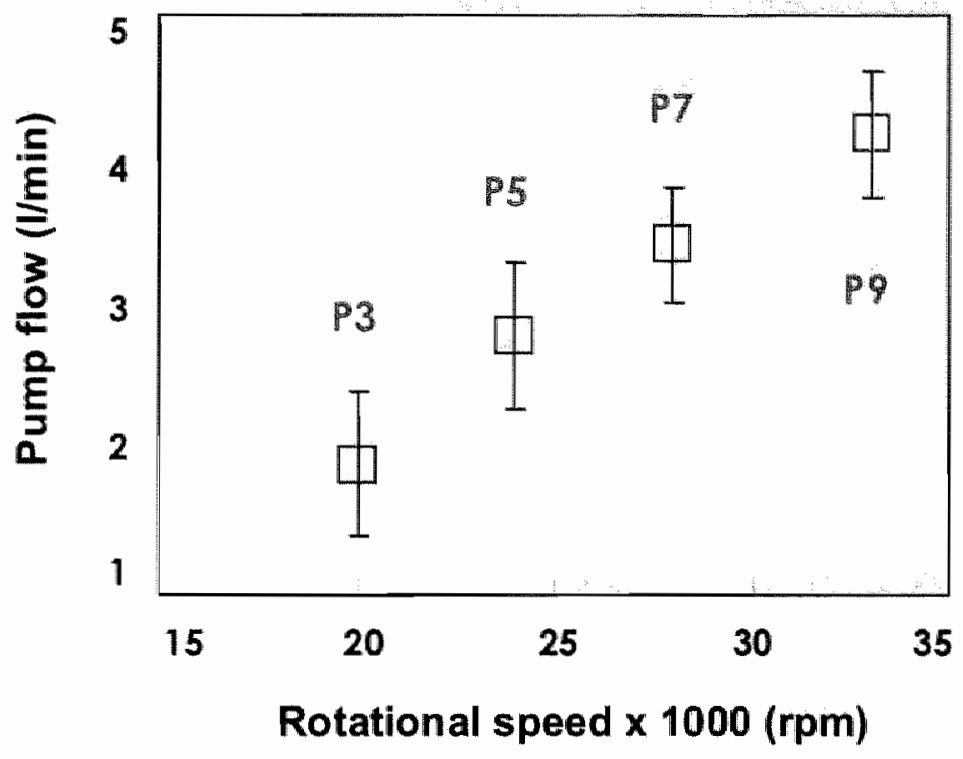

Figure 6.1 Impella pump flow characteristic (means \pm SD) during mitral regurgitation (pressure gradient across the pump $<50 \mathrm{~mm} \mathrm{Hg}$ ). The performance $\left(P^{3}\right)$ levels are related to the pump's average rotational speed by definition.

\section{RESULTS}

\section{Pump characteristics}

Figure 6.1 shows the performance of the IMP measured in vivo. The measurements were done during acute mitral regurgitation and at four different performance levels. The pump flow values were taken from the console's flow reading. Average pump flow has a linear relationship to the selected totational speed and ranges from 1.9 to $4.2 \mathrm{l} / \mathrm{min}$. The standard deviation remains fairly constant at $0.5 \mathrm{l} / \mathrm{min}$ throughout the range. 
106 | Chapter 6

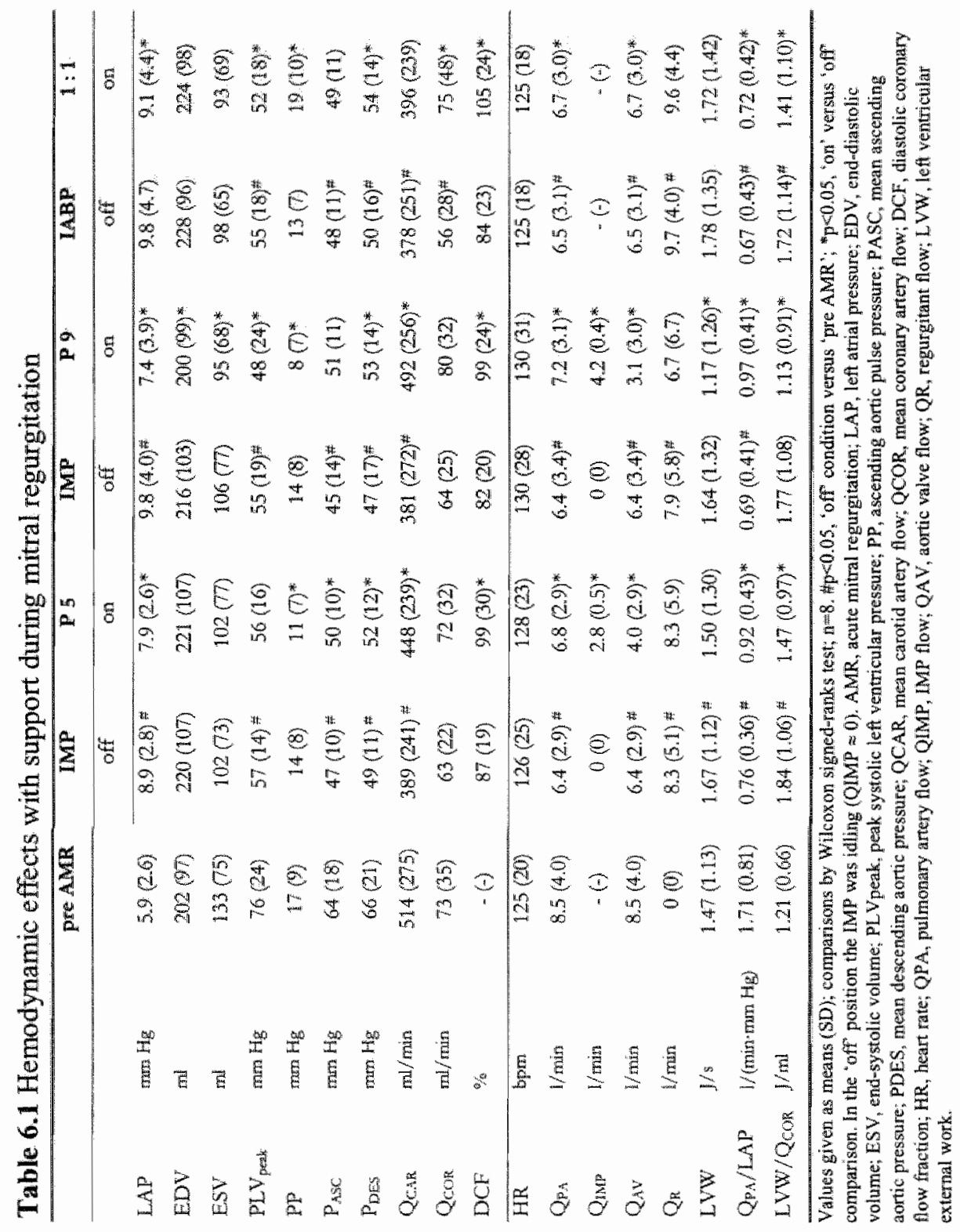




\section{Hemodynamic and functional effects}

The effects of support on hemodynamics and cardiac function are summarized in Table 6.1. Only the IMP in P9 mode significantly reduced both end-diastolic and end-systolic left ventricular volume (EDV $-7 \%$ and ESV -10\%). The IMP had a greater effect $(-7 \mathrm{~mm} \mathrm{Hg})$ than IABP assist $(-3 \mathrm{~mm} \mathrm{Hg})$ on peak systolic left ventricular pressure. Ascending aortic pulse pressure (PP) was significantly reduced by the IMP with a maximum reduction of $6 \mathrm{~mm} \mathrm{Hg}$ at P9. In contrast, IABP increased PP by $6 \mathrm{~mm} \mathrm{Hg}$. Mean carotid artery flow was augmented significantly by the IMP only $\left(\mathrm{Q}_{\mathrm{CAR}}+15 \%\right.$ and $\left.+29 \%\right)$ while mean coronary flow was only enhanced significantly during IABP $\left(\mathrm{Q}_{\mathrm{COR}}+34 \%\right)$. Forward cardiac output (pulmonary artery flow) was increased significantly by both IABP $(+3 \%)$ and the IMP (P5, $+6 \%$ and P9, $+13 \%)$.

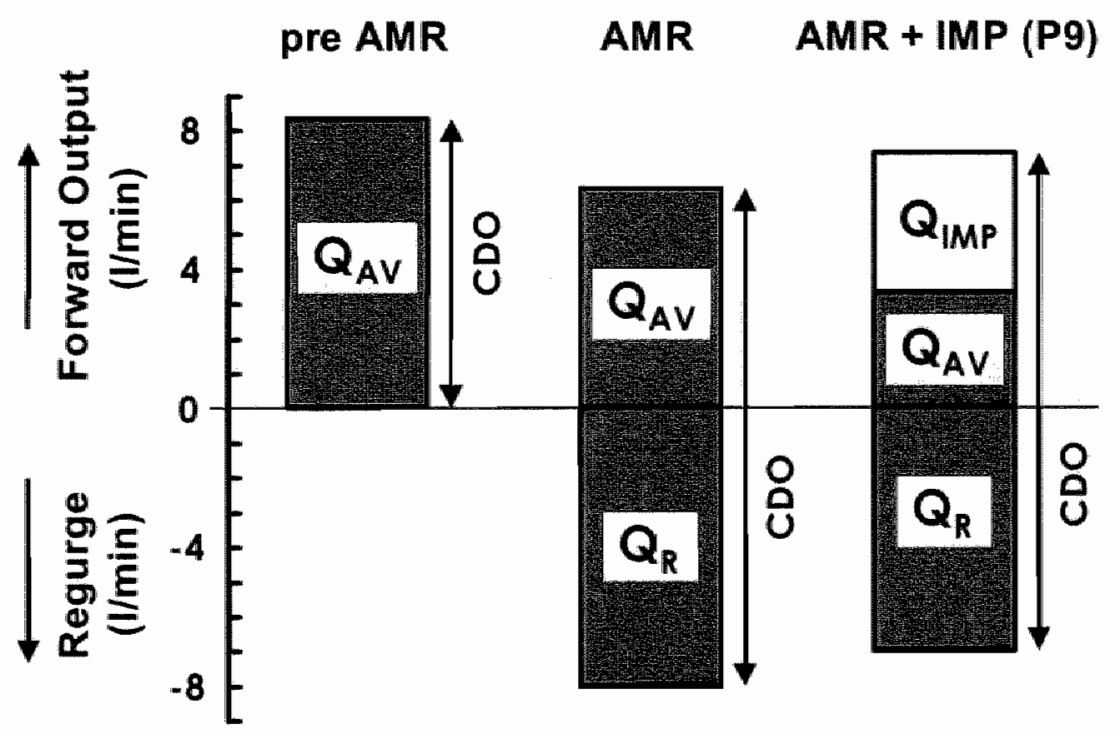

Figure 6.2 Composition of forward output and conductance derived output before AMR, during AMR, and with IMP (P9) support. QAv, flow through the aortic valve; QIMP, IMP flow; QR, regurgitant flow; CDO, conductance derived output. Note that with IMP support CDO contains QMarp, and that not the absolute value but the direction (forward/regurge) of CDO changes. 
108 | Chapter 6

Eigure 6.2 defines the various modes of flow. The height of the columns corresponds with the mean values stated in Table 6.1. IABP increased aortic valve flow $\left(\mathrm{Q}_{\mathrm{AV}}\right)$ by $3 \%$, whereas the IMP reduced $\mathrm{Q}_{\mathrm{AV}}$ by $-38 \%$ and $-52 \%$ in the P5 and P9 mode respectively (Table 6.1). Regurgitant flow through the mitral valve was reduced to some extent in the IMP P9 setting only $\left(Q_{R}-15 \%, p=N S\right)$. External left ventricular work was only significantly reduced with full IMP support (P9, LVW -29\%). The index for left heart pump function $\left(\mathrm{Q}_{\mathrm{PA}} / \mathrm{LAP}\right)$ significantly increased with assist (IMP $+21 \%$ and $+41 \%$, IABP $+7 \%$; Table 6.1$)$.

In Figure 6.3 the effects on left heart pump function are further analysed by incorporating the pre assist hemodynamic condition per individual. Pre assist forward cardiac output ranged from 2 to $12 \mathrm{l} / \mathrm{min}$ (i.e. after induction of mitral regurgitation). The trends indicate that support is

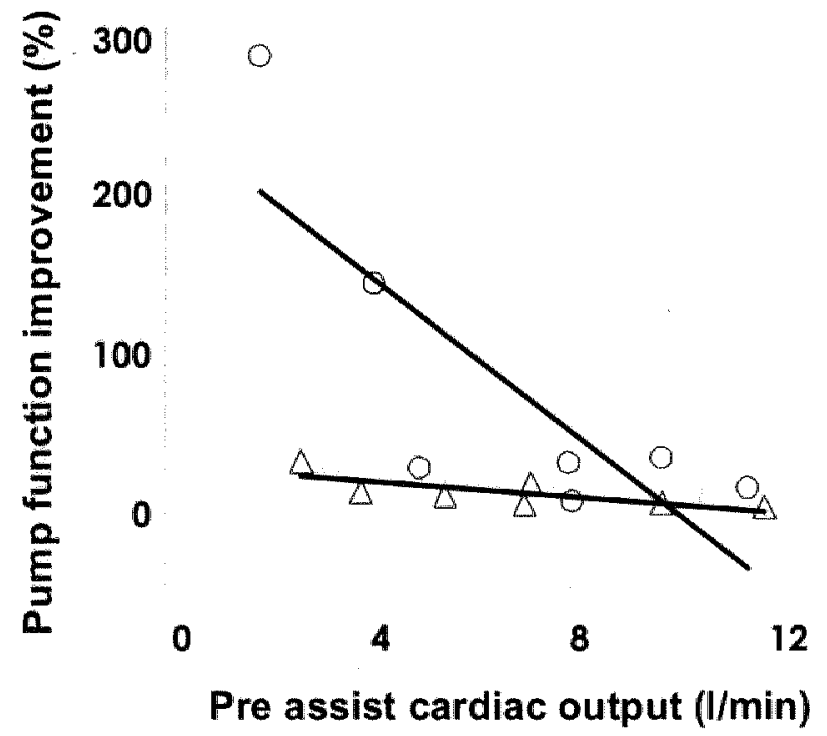

Figure 6.3 Improvement of left heart pump function $\left(\mathrm{QpA}_{\mathrm{p}} / \mathrm{LAP}_{\mathrm{A}}\right)$ per individual animal. Open circles, IMP support (P9); triangles, IABP support (1:1). Differentiation between animals is on the basis of pre assist cardiac output (horizontal axis). Vertical axis: Relative change (\%) of the $\mathrm{QPA}_{\mathrm{PA}} / \mathrm{AP}$ index as a result of assist. 


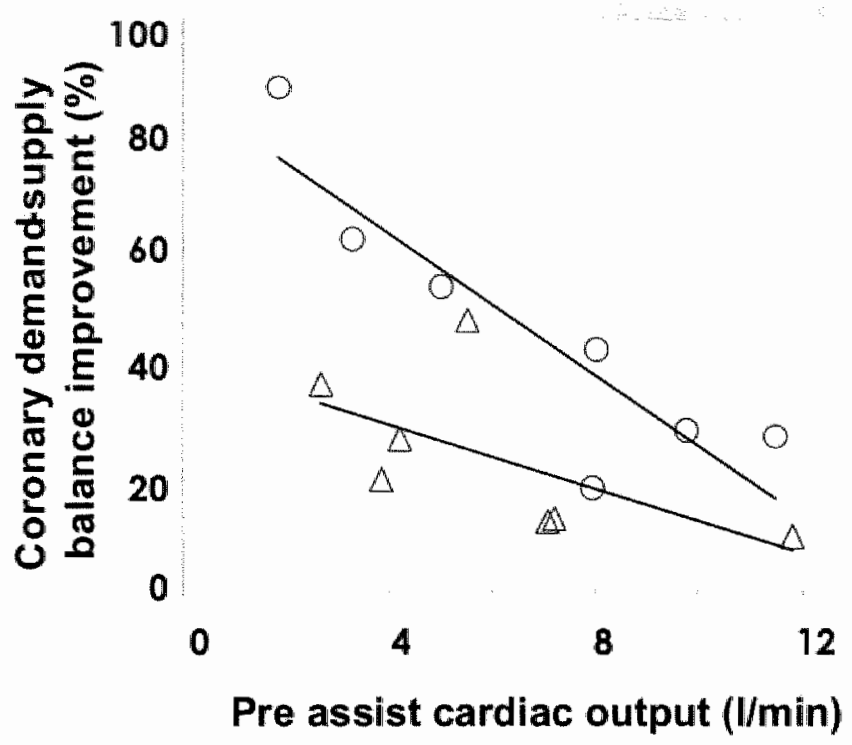

Figure 6.4 Improvement of the coronary demand-supply balance (LVW/Qcon) per individual animal. Open circles, IMP support (P9); triangles, IABP support (1:1). Differentiation between animals is on the basis of pre assist cardiac output (horizontal axis). Vertical axis: -Relative change (\%) of the LVW/Qcor index as a result of assist. (Decrease of LVW/QCor is an improvement in the demand-supply balance.)

more effective with lower pre assist cardiac outputs. The difference in effectiveness between IABP and the IMP increases with the same trend. Table 6.1 also shows the LVW/QCor index decreased significantly in the 1:1 IABP $(-18 \%)$, and P5 and P9 IMP modes $(-20 \%$ and $-36 \%)$.

Figure 6.4 focuses on the coronary demand-supply balance improvement per individual. Note that a decrease in the LVW/Q corresponds to an improvement in coronary demand-supply ballance.)

\section{Hemodynamic trends}

Among the subjects that had a pre assist cardiac output lower than $41 / \mathrm{min}$, pronounced effects on pump function and coronary balance were seen 
(Figs. 6.3 and 6.4). Figure 6.5 shows paired trends (obtained from one single animal) that are typical of the full $\mathbb{L V}$ bypass situation observed in these particular subjects. After switching on P9 IMP support, peak systolic LV pressure progresses below even diastolic aortic pressure levels. Apparently, during systole there is no longer a forward pressure gradient and therefore no flow through the aortic valve $\left(Q_{A V}=0\right.$, with reference to Fig. 6.2).

Peak ascending aortic pressure and pulsatility are increased by IABP. The IMP increases mean ascending aortic pressure while it reduces pulsatility. The coronary flow recordings show that both pumps enhance primarily diastolic coronary flow. This is sustained by the results on diastolic coronary flow fraction (DCF) as given in Table 6.1.

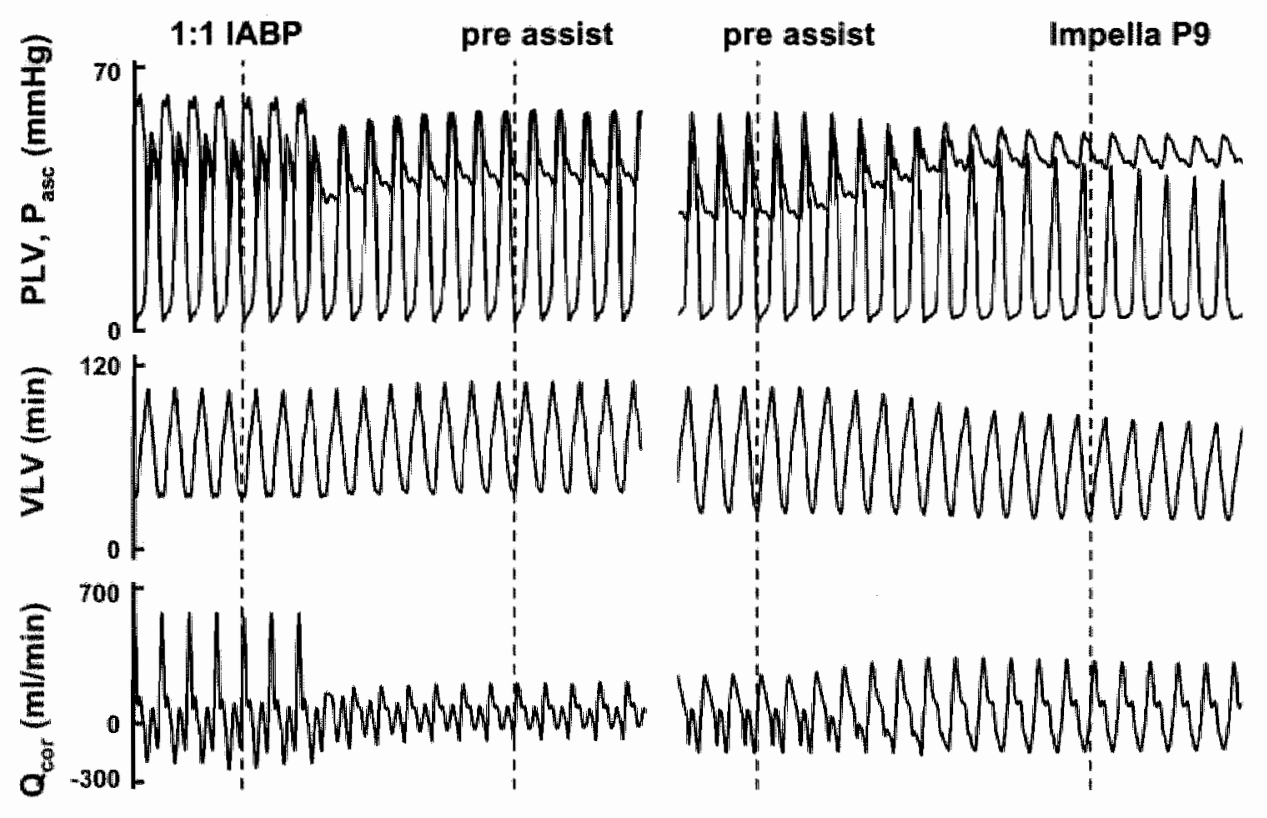

Figure 6.5 Specific hemodynamic trends (obtained from one animal) that illustrate the full LV bypass condition seen in some animals with full IMP support. Note that the assist on/off sequence is different in both panels. $P_{A S C}$, ascending aortic pressure; PLV, left

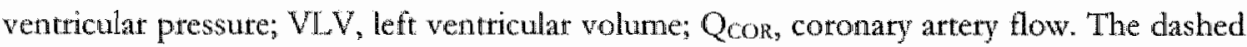
lines indicate the start of diastole. 


\section{DISCUSSION}

\section{Circulatory support}

The reduction of elevated left atrial pressures, indicative of mitral regurgitation, implies that pulmonary venous congestion is suppressed, which may counter the development or further progress of lung edema. Peripheral perfusion was significantly improved, which shows that both assist systems do provide circulatory support in the event of left heart failure (Table 6.1).

\section{Cardiac unloading}

The reduction of work and oxygen consumption is an essential element of cardiac unloading and cardio-protective therapy. Afterload is a major determinant of myocardial work ${ }^{1-18}$. PLV peak as a measure of left ventricular afterload was reduced with assist (Table 6.1) ${ }^{19}$. In gencral, a suitable definition of left ventricular pressure load would be $\left(\mathrm{P}_{\mathrm{ASC}}-\mathrm{LAP}\right)^{20}$, but this factor is prone to error in the case of left ventricular and aortic pressure dissociation (Fig. 6.5). Therefore, external left ventricular pressure work was estimated from (PLV $\left.{ }_{\text {peak }}-\mathrm{LAP}\right)$.

The IMP, in P9 mode, reduced both regurgitant and aortic valve flow $(-15 \%$ and $-52 \%$ ), as well as external work (LVW $-29 \%$ ) and LV volume (EDV - $7 \%$ and ESV - $10 \%$ ). These data suggest that during full IMP support total left ventricular work and myocardial oxygen consumption are reduced ${ }^{18,21,22}$. IABP showed less effective unloading capabilities.

\section{Myocardial support}

In general, myocardial function is supported by reducing myocardial work and augmenting coronary perfusion. The IMP improved coronary perfusion $\left(Q_{C O R}+25 \%\right)$ and strongly reduced cardiac workload (LVW $-29 \%$ and 
$\left.\mathrm{PLV}_{\text {peak }}-13 \%\right)^{18}$. IABP improved primarily coronary perfusion (Q $+34 \%$ ) as work reduction was only marginal (LVW $-3 \%$ and $\mathrm{PLV}_{\text {peat }}-5 \%$ ). The coronary demand-supply balance is significantly improved (i.e. LVW/Qcor decreased) by both (Table 6.1), but the overall effectiveness of the IMP is greater than IABP (Fig. 6.3). The LVW/ $\mathrm{Q}_{\mathrm{Cor}}$ ratio reflects both the ventricular unloading and coronary perfusion aspects. In clinical practice, priority may lie with either one of these. If priority lies with myocardial oxygen supply (control of ischemia), IABP remains an effective, well-proven therapy ${ }^{23}$. When additional left ventricular unloading and immediate circulatory support are mandatory, the IMP may present a solid alternative.

\section{Impact of pre assist condition}

Analysis of the effect of support between individual subjects shows that functional improvements with assist are greater in weaker subjects (Figs. 6.3 and 6.4). In the P9 setting average IMP flow was $4.2 \mathrm{l} / \mathrm{min}$ (Fig. 6.1). With pre assist cardiac outputs greater than $8 \mathrm{l} / \mathrm{min}$ a bypass flow of $4 \mathrm{l} / \mathrm{min}$ naturally has only limited impact. In individuals with insufficient cardiac output $(<4 \mathrm{l} / \mathrm{min})$ pump function improvement was more pronounced (IMP greater than $+100 \%$ and up to $+25 \%$ with IABP; Fig. 6.3). These trends and the data in Table 6.1 suggest that the IMP has greater effectiveness in improving overall pump function than IABP. Figure 6.4 shows that IABP partially compensates for its poor volume unloading performance by improving the coronary perfusion aspect ${ }^{1,5,9}$.

\section{Left ventricular output and IMP output}

Table 6.1 states forward output $\left(Q_{p N}\right)$, regurgitant flow $\left(Q_{R}\right)$, and aortic valve flow $\left(\mathrm{Q}_{\mathrm{AV}}\right)$. Figure 6.2 provides a detailed overview of the left ventricular and device related contributions to forward cardiac output. Apparently, $C D O$ is the sum of $Q_{R}, Q_{A v}$ and $Q_{I M p}$. The slight discrepancy found when calculating CDO from Table 6.1 is due to rounding errors 
(EDV - ESV) $\cdot H R \cong 13.7 \mathrm{l} / \mathrm{min}$ while $\left.Q_{R}+Q_{A N}+Q_{\text {inp }} \cong 141 / \mathrm{min}\right)$. Output analysis shows that part of the stroke volume ejected by the $I V$ is via the IMP: CDO contains $\mathrm{Q}_{\text {mp }}$ (Fig. 6.2). The flow through the IMP ( $\mathrm{Q}_{\mathrm{IMP}}$ ) is due to the simultaneous effect of $\mathrm{LV}$ contraction and IMP aspiration. Essentially, the IMP acts as an afterload reduction device that augments ventricular output towards the aorta, which effectively redistributes forward output and regurgitant flow. (The cumulative bas is lifted with respect to the zero output level in Figure 6.2.)

LVW was calculated from $\left[P L V_{\text {peak }}-L A P\right] \cdot\left[Q_{A V}+Q_{n}\right]$, which implies that the fraction of LV stroke output through the IMP does not contribute to external LV work. Realistically, this assumption may introduce some error. While both the LV and IMP work simultaneously, the exact share of pump and cardiac work can not be identified.

\section{Pulsatility}

Whereas the IMP reduces the pulsatility in the aorta, IABP increases pulse pressure (Fig. 6.5 and Table 6.1). Evaluation of the quality of pulseless perfusion solely based on hemodynamic measurements is controversial and is therefore not further addressed ${ }^{24}$. Moreover, the present study is focused on acute hemodynamic collapse, which renders long term pulseless perfusion effects less relevant. Interestingly, however, the IMP did not flatten the coronary flow curve, but exhibited a behavior comparable to LABP during systole (Fig. 6.5). With IABP decreased systolic flow can be attributed to the intentional aortic pressure reduction at end-diastole. The decrease in systolic flow with the IMP may be caused by a pressure decline due to an accelerated jet (Venturi effect) from the IMP's outflow ${ }^{25}$.

\section{Mode1}

Other investigators who tested a device similar to the IMP reported less promising results ${ }^{20}$. In their bovine ischemic LV dysfunction model (coronary ligation) mean aortic pressures of $88 \mathrm{~mm} \mathbb{H g}$ existed in the 
presence of a cardiac inder (Cl) of $2.11 /\left(\min \cdot \mathrm{m}^{2}\right)$. (This study: Aartic pressure $<50 \mathrm{~mm} \mathrm{Hg}$; CI $3.2 \mathbb{1} /\left(\mathrm{min} \cdot \mathrm{m}^{2}\right)$.) As high afterload limits the efficacy of axial flow pumps, insufficient hemodynamic collapse will not challenge these devices. As the IMP was designed to provide support up to $4.5 \mathrm{l} / \mathrm{min}$, a substantial level of hemodynamic compromise is considered

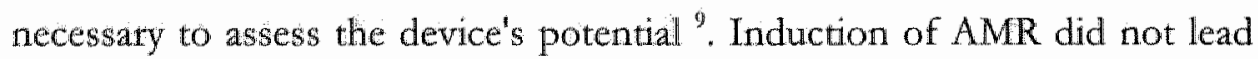
to substantially elevated left atrial pressures ${ }^{5}$. The pre AMR values of $Q_{P A}$ and $P_{A S C}$ in Table 6.1 could be indicative of a distended peripheral arteriolar vascular bed at baseline. Vasodilation is known to reduce the effects of mitral regurgitation ${ }^{26}$. Ultimately, the level of hemodynamic compromise obtained in the present study did prove effective to characterize the IMP and IABP.

\section{Clinical implications}

The present results suggest that the IMP can effectively reduce the cardiac workload while increasing cardiac output and that it outperforms IABP in low cardiac output conditions. This implies that the IMP may be a better alternative in circulatory collapse patients ${ }^{4,27}$. Moreover, in the critical situation of sudden left heart deterioration, the IMP may provide a safety net, buying time for the medical team to act.

Though the present study has illustrated potential of the IMP to take over the left heart pump function (Fig. 6.5), it must be realized that the pumping capacity heavily depends on the availability of preload. Right-sided circulatory failure seriously limits the effectiveness of left ventricular assist devices and is a contraindication as such ${ }^{28,29}$.

In the event of unstable angina both the IMP and IABP would be beneficial in terms of sustained afterload reduction and coronary perfusion enhancement ${ }^{23}$. As timing and synchronization are critical with IABP, the continuous flow IMP might perform more consistently than IABP in rhythmically unstable patients (e.g. atrial fibrillation).

The IMP is a $6.4 \mathrm{~mm}$ diameter (19 French) device which requires surgical implantation under direct vision. A smaller version of the IMP 
would allow easier percutaneous introduction and might stretch the indications for intracorporeal support beyond those for LABP. Moreover, both systems could prove synergetic when applied simultaneously.

\section{Conclusion}

We studied the hemodynamic efficacy of an intracardiac microaxial pump and intra-aortic balloon pumping in a bovine model of acute mitral regurgitation. We conclude that in more severely compromised subjects, and in the event of sudden decompensation due to severe acute left ventricular pump failure, the intracardiac microaxial pump is capable of more effective cardiac and circulatory support than intra-aortic balloon pumping.

\section{References}

1 Weber KT, Janicki JS. Intraaortic balloon counterpulsation. A review of physiological principles, clinical results, and device safety. Ann Thorac Surg $1974 ; 17(6): 602-36$

2 Kantrowitz A, Cardona RR, Freed PS. Percutaneous intra-aortic balloon counterpulsation. Crit Care Clin 1992;8(4):819-37

3 Mueller HS. Rolle of intra-aortic counterpulsation in cardiogenic shock and acute myocardial infarction. Cardiology 1994;84(3):168-74.

4 Hollenberg SM, Kavinsky C], Parrillo JE Cardiogenic shock. Ann Intern Med $1999 ; 131(1): 47-59$

5 Dekker AL, Reesink KD, Van der Veen FH et al. Intra-aortic balloon pumping in acute mitral regurgitarion reduces aortic impedance and regurgitant fraction. Shock $2003 ; 19(4): 334-8$

6 Lonn $U$, Peterzen B, Granfeldt $H$ et al. Hemopump treatment in patients with postcardiotomy heart failure. Ann Thorac Surg 1995;60(4):1067-71

7 Nishimura $Y$, Meyns B, Ozaki S et al. The enabler cannula pump: a novel circulatory support system. Int I Artif Organs 1999;22(5):317-23

8 Dekker AL, Geskes GG, Cramers AA et al. Right ventricular support for offpump coronary ártery bypass grafuing studied with bi-ventricular pressurewolume loops in sheep. European Journal of Cardio-thoracic Surgery 2001;19:179-84 
9 Dekker AL, Reesink KD, Van der Veen FH et al. Efficacy of a new Intra-aortic Propeller Pump versus the Intra-aortic Balloon Pump: an animal study. Chest $2003 ; 123: 2089-95$

10 Autschbach $\mathbb{R}$, Rauch $T$, Engel M et al. A new intracardiac microaxial pump: first results of a multicenter study. Artificial Organs 2001;25(5):327-30

11 Meyns $B$, Autschbach $R$, Boning A er al Coronary artery bypass grafting supported with intracardiac microaxial pumps versus normothermic cardiopulmonary bypass: a prospective randomized trial European Journal of Cardio-Thoracic Surgery 2002;22(1):112-7

12 Reul HM, Akdis M. Blood pumps for circulatory support. Perfusion 2000; 15:295311

13 Siess T, Nix C, Menzler F. From a lab type to a product: a retrospective view on Impella's assist technology. Artificial Organs 2001;25(5):414-21

14 Steendijk P, Van der Veide ET, Baan J. Left ventricular stroke wolume by single and dual excitation of conductance catheter in dogs. Am J Physiol $1993 ; 264: 112198-207$

15 Baan J, Van der Velde ET, De Bruin HG et al. Contimuous measurement of left ventricular volume in animals and humans by conductance catherer. Circulation. $1984 ; 70: 812-23$

16 Amirthamzeh MM, Dean DA, Jia CX et al. Validation of right and left ventricular conductance and echocardiography for cardiac function studies. Ann Thorac Surg $1996,62(4): 11049$

17 Guyton AC, Hall JE. Textbook of Medical Physiology. 9th ed. Philadelphia: W.B. Saunders Company, 1996:116

18 Suga $H$, Hisano R, Hitata S et al. Mechanism of higher oxygen consumption rate: pressure-londed vs. volume-loaded heart. Am J Physiol 1982;242(6):H942-8

19 Notton JM. Toward consistent definitions for preload and afterload. Advances in Physiology Education 2001;25(1):53-61

20 Martks JD, Pantalos GM, Long JW et al. Myocardial mechanics, energetics, and hemodynamics during intraortic balloon and transvalvular axial flow hemopump support with a bovine model of ischemic cardiac dysfunction. Asaio I $1999,45(6): 602-9$

21 Suga H. Total mechanical energy of a ventricle model and cardiac oxygen consumption. Am J Playsiol 1979;236(3):H498-505

22 Teien DE, Jones $M$, Shiota $T$ et al. Jeft ventricular stroke work in mitral regurgitation: an animal experimental study. J Heart Valve Dis 1997;6(6):613-20

23 Torchiana DF, Hirsch $G$, Buckley MJ et al. Intraaortic balloon pumping for cardiac support: trends in practice and outcome, 1968 to 1995. J Thotac Cardiovasc Surg 1997;113(4):758-69 
24 Honick P, Taylor K. Pulsatile and nonpulsatle pertission: the continuing controversy. J Cardiothorac Vasc Anesth 1997;11(3):310-5

25 Van Ommen VG, van den Bos AA, Pieper M et al, Renoval of dhrombus from aortocoronary bypass grafts and coronary arteries using the 6Fr Hydrolyser. Am ) Cardiol 1997;79(8):1012-6.

26 Yoran C, Yellin EL, Becker RM et al. Mechanism of reduction of mitual regurgitation with vasodilator therapy. Am J Cardiol 1979;43(4):773-7

27 Nishimuta RA, Schaff HV, Shub C et al. Papillary muscle rupture complicaring acute myocardial infarccion: analysis of 17 patients. Am J Cardiol 1983;51(3):373-7

28 Fartat D, Compton P, Hershon J et al. Right ventriculat function in an operating room model of mechanical left ventricular assistance and its effects in patients with depressed left ventricular function. Circulation 1985;72(6):1279-85

29 Chen JM, Rose EA. Management of periooperative right-sided circulatory failure. In: Goldstein DJ; Oz M, editors. Cardiac Assist Dewices. Armonk, NY: Futura Publishing, 2000:83-101 
$118 /$ Chapter 6 
LVAD suction | 119

\section{Chapter 7}

\section{Circulatory instability due to left ventricular assist device suction: Cause and remedy}

\section{Submitted as:}

Reesink KD, Dekket ALAJ, wan der Nagel T, Beghi C, Leonardi $F$, Botti P, De Cicco $\mathrm{G}_{\text {, }}$ Lorusso R, Schreuder JJ, van der Veen $\mathrm{FH}_{3}$ Maessen JG. Left ventriculat assist device suction: implications for device control and management. 


\section{ABSTRACT}

The current generation of left ventricular assist devices (LVADs) uses rotary impeller technology which promises features such as longevity, reliability, and biocompatibility. However, the physiological responsiveness of rotary blood pumps is inherently low, which may lead to hemodynamic imbalance, when such a device is used to assist the circulation. Such an imbalance is manifest during pump inlet suction which may cause excessive hemolysis, cavitation, and reduced device efficacy and efficiency. Little evidence is available, on how suction affects the circulatory function, and which factors determine its occurrence and the persistence of the associated hemodynamic collapse.

We investigated the mechanism of suction in two distinct hemodynamic models of the assisted circulation. Suction was intentionally elicited in 6 sheep during rotary LVAD support. A computer simulation protocol was conducted in parallel.

The results indicate that LVAD support is capable of unloading the pulmonary circulation and the right heart, in addition to bypassing the left heart. The efficacy of unloading was shown to depend on circulatory filling, right heart function, and pulmonary vascular condition. After an initial fitting iteration, the computer simulation model showed good correlation with the in vivo data. The dynamic evaluation of suction induced hemodynamic collapse and the subsequent recovery from it, revealed a nonlinear relationship: Depending on the existence of a predisposing condition, even a short period of suction could induce a prolonged state of persistent collapse. The contimuous and forced aspiration of the LVAD was a primary determinant of this behavior: Swift resolution of the collapsed state could be brought about by a transient pump shut down intervention.

Integrative analysis of the body of data shows, that the observed behavior can be explained by the concept of a suction induced starling resistor positioned at the site of vessel collapse. This concept sustains the gross effects of full suction, as well as the congestive character of imminent or 'silent' suction. 


\section{INTRODUCTION}

The left ventricular assist device (LVAD) has gained widespread use as a surgical treatment modality for heart failure patients and is employed to bridge patients to corrective surgery, heart transplantation, and recovery ${ }^{1.5}$. The use of the LVAD as a permanent support modality is considered feasible but remains under continuous investigation ${ }^{6-8}$. Major complications of LVAD support are: Infection, thrombo-embolism, device failure, and right-sided circulatory failure (RSCF). RSCF constitutes the more obscure and complex comorbidity in 15-30\% of LVAD recipients ${ }^{7.12}$. Both beneficial and deleterious effects of left ventricular assist on the right heart and pulmonary function have been reported 1.4,6,9,11.

A well-adjusted balance in pumping action, volume management, and medication are important to obtain a positive effect with LVAD support. The latest generation of assist devices is of the aspirator pump type. These devices produce a continuous flow and therefore lack inherently the typical sensitivity to preload of the native ventricle. As a result, these pumps need continuous adjustment or automatic feedback control to avoid over-aspiration which is associated with hemolysis, thrombus release, and tissue damage at the pump inlet ${ }^{12-18}$. When suction events do not occur, the patency of LVAD support is generally considered guaranteed based on the premise that the backward system (the pulmonary circulation) will comply. Little experimental and clinical data is available on the mechanics of suction. Furthermore, the impact of imminent or actual suction on the pulmonary circulatory function has not been investigated to great extent.

In the present study, we have intentionally elicited suction in two distinct hemodynamic models to scrutinize the determinants and predispositions involved. Through the analysis of LVAD aspiration and suction we have developed a mechanistic perspective on the probable causes and the role of RSCF in LVAD patients. 
$122 \mid$ Chaprer 7

\section{METHODS}

The experimental protocol was conceived as a pilot to obtain preliminary data. Computer simulations were then employed to further detail the experimental results.

\section{Experimental protocol}

\section{Animal preparation}

All animals received humane care in compliance with the "Guide for the Care and Use of Laboratory Animals" (NIH publication 86-23, 1985 revision; National Institutes of Health, USA). Six Sardinian sheep weighing $49 \pm 3 \mathrm{~kg}$ (mean \pm SD) were premedicated with acetylptomazine $(0.05$ $\mathrm{mg} / \mathrm{kg} \mathrm{i} . \mathrm{m}$.) and xylazine $(0.05 \mathrm{mg} / \mathrm{kg}$ i.m.). Anesthesia was induced with sodium pentothal (bolus $20 \mathrm{mg} / \mathrm{kg}$ i.v.) and maintained with a $1: 2$ mixture of $\mathrm{O}_{2}: \mathrm{N}_{2} \mathrm{O}$ and halothane (1.5-2.0\%). After administration of analgesic fentanyl (bolus $0.1 \mathrm{mg} / \mathrm{kg}$ i.v.) and muscle relaxant pancuronium bromide (bolus $0.1 \mathrm{mg} / \mathrm{kg}$ i.v.), a left thoracotomy was performed while maintaining a peak end-expiratory pressure (PEEP) of $5 \mathrm{~cm} \mathrm{H} \mathrm{H}_{2} \mathrm{O}$. Fluid management was through continuous infusion of Ringer's lactate $(10-20 \mathrm{ml} / \mathrm{kg} / \mathrm{h}$ i.v.). Coagulation was controlled through administration of heparin (bolus 200 $\mathrm{IU} / \mathrm{kg}$ i.v.). Monitoring included ECG, blood pressure, oxygen saturation and capnography. The animal was sacrificed through a pentobarbital overdose (bolus $80-100 \mathrm{mg} / \mathrm{kg}$ i.v.). The study was approved by the ethical committee of the University of Parma, Italy.

\section{Mechanical circulatory support systems}

Each animal received a rotary left ventricular assist device of the radial/centrifugal type (Arrow International, Reading, PA) ${ }^{18}$. The inflow extension of the pump was inserted into the left ventricular apex and the outflow graft was anastomosed to the aortic arch. The LVAD was powered and controlled by its proprietary power supply and notebook controller. Pump operation monitoring included rotary speed (set level and actual), estimated pump flow, and motor current ${ }^{18}$. 
A cardiopulmonary bypass circuit was used during partial aortic clamping. Arterio-venous $\mathrm{CPB}$ access was via $21 \mathrm{Fr}$ carotid and $24 \mathrm{Fr}$ jugular cannulae. The circuit's priming solution consisted of Hemacell, Ringer's lactate, and heparin. During prolonged CPB overall anesthetic maintenance was preserved by periodic Propofol infusion. Circulatory volume was controlled with the aid of the venous reservoir and guided by tight ventricular diastolic and aortic pressure monitoring.

\section{Instrumentation}

A Swan-Ganz thermodilution catheter with integrated tip sensor (Arrow International, Reading, PA) was advanced into the pulmonary artery via the left femoral vein. An additional solid-state pressure catheter (CD Leycom, Zoetermeer, the Netherlands) was introduced via the right femoral vein and positioned in the right ventricle. Two solid-state pressure catheters were placed in the ascending aorta and left ventricle via the femoral arteries. A left atrial solid-state pressure catheter was placed via a purse-string suture. Ultrasonic flow probes (Transonic Systems, Ithaca, NY) were placed on the pulmonary artery and on the outflow graft of the LVAD. A retractable tourniquet was placed on the proximal pulmonary artery to enable acute occlusion interventions.

\section{Measurements and analysis}

The steady-state hemodynamic effects of LVAD operation were evaluated during three conditions: Baseline (LVAD off and outflow graft occluded), minimum rotary speed (2200 rpm), and maximum rotary speed (3000 rpm). Suction was evaluated in a three step approach:

Full pulmonary artery occlusions (PAOC) were performed under LVAD off and on conditions. In selected subjects different durations of occlusion were obtained. Transient manipulations in LVAD rotational speed were made to evaluate the dynamic response of the circulation. If (impending) suction was noted upon a speed intervention, the adjustment was reversed subsequently to avoid full suction. To evaluate the influence of thoracic extravascular pressures on pulmonary flow during LVAD support (as a model for e.g. mechanical ventilation, coughing and sneezing, and 
Valsalva maneuver) manual lung ventilation was employed. The intervention included two ventilatory cycles within a 10 second episode.

All data were stored on a portable data acquisition system (IDEEQ, IDEE Instrumentation Services, University of Maastricht, The Netherlands) featuring a 16 channel, 12 bit PCMCIA A/D-converter (Keithley Instruments, Cleveland, $O H)$. Statistical comparisons included nonparametric Wilcoxon signed-ranks tests $(n=6)$. Significance was assumed if $\mathrm{p}<.05$.

\section{Computer simulations}

\section{Model description}

We developed a proprietary hemodynamic model of the circulation by implementing standard physiological data in an electronic circuit simulation program (PSpice, Cadence Design Systems, San Jose, CA) ${ }^{19}$. The model consists of four subsystems that are serially connected to form a loop (Fig. 7.1).

The partial models for the right and left heart contain: (1) a passive ventricular compliance in series with a preload sensitive systolic pressure source to simulate a contractile ventricle, (2) a finite capability to increase contraction force as demanded by preload, (3) a passive compliance for an atrium, and (4) gradient controlled switches for the valves $12,13,20$.

The vascular beds are structurally divided into proximal, central, and distal segments which feature lumped linear resistances and compliances. The central segments include additional gradient dependent resistors that mock starling resistor behavior and pressure homeostat response ${ }^{12,13,20-24}$. The extravascular nodes of each intra-thoracic compliance are connected to a pressure source which provides a negative intra-thoracic pressure of -4 $\mathrm{mm} \mathrm{Hg}$. Periodic variations of $+/-.2 \mathrm{~mm} \mathrm{Hg}$ and frequency $6 \mathrm{bpm}$ are superimposed to mock the respiratory impact on vascular volume and cardiac filling ${ }^{13,20,25}$. Circulatory volume is set to 5.7 liters. 

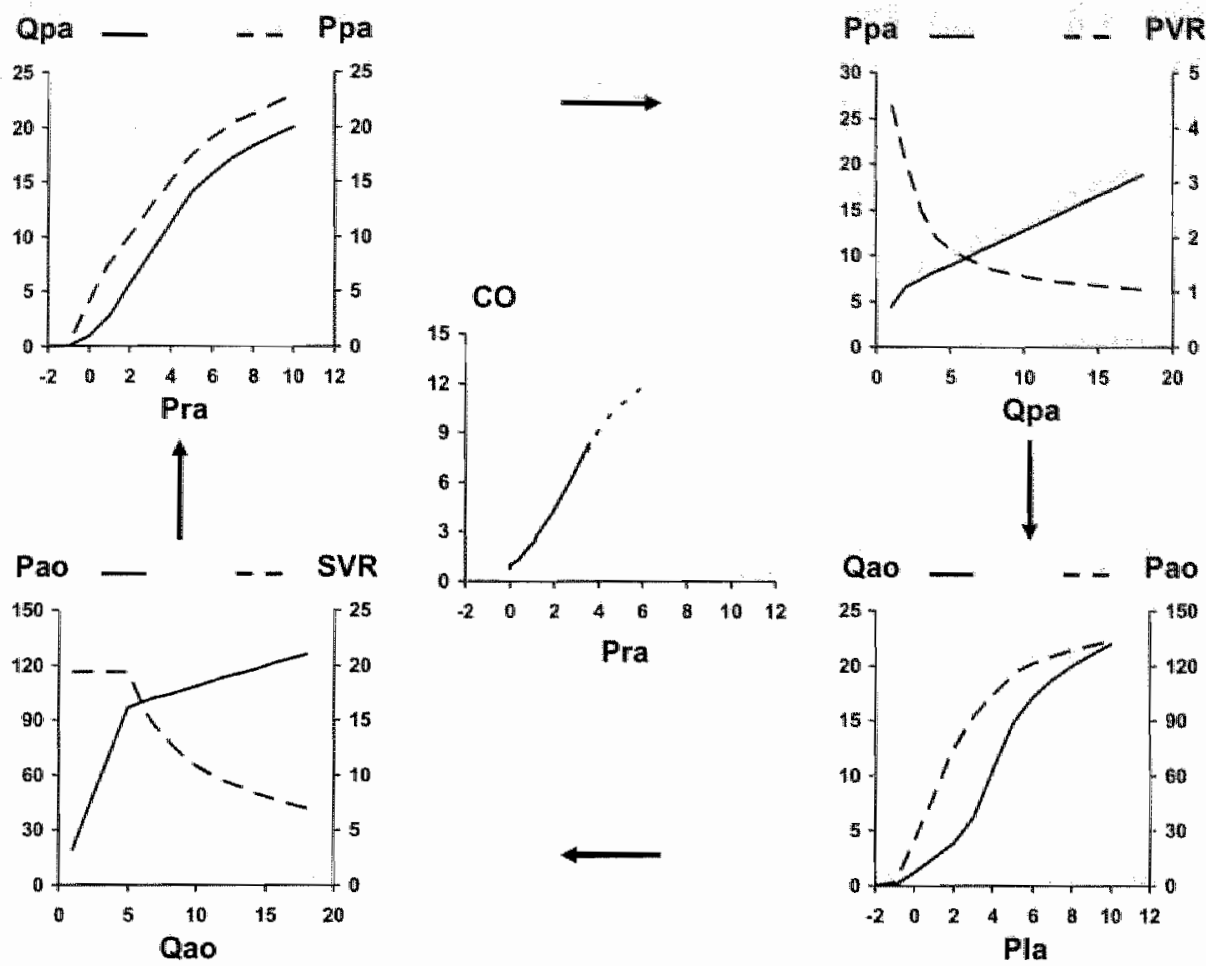

Figure 7.1 Functional diagram of the circulation simulation model. The subsystems are characterized by their respective input-output relationships. From the top-left, clockwise: Right heart, pulmonary wascular bed, left heart, and systemic vascular bed. In the center the aggregate response to venous return is presented in the form of a cardiac output curve. (Hetein the extrapolated portion was obtained with increased circulatory filling.) Qpa, pulmonary artery flow; $\mathrm{Ppa}$, pulmonary arterial pressure; Pra, tight atrial pressure; PVR, pulmonary vascular resistance; Qgo, aottic flow; Pao, aortic pressure; Pla, left atrial pressure; $C O$, cardiac output; SVR, systemic vascular resistance. All values along the axes: Pressures in $\mathrm{mm} \mathrm{Hg}$ flows in $\mathrm{l} / \mathrm{min}$.

Specific model elements that are included are: A lossy continuous flow source to model the LVAD, and a transmural pressure gradient controlled switch to model a collapsible vessel immediately preceding the mitral. valve element ${ }^{12,13,26}$.

The input-output relationships of the pulmonary and systemic vascular beds were obtained by simulating an in vivo physiology lab setup for isolated characterization. The vascular beds were perfused by an ideal 
continuous flow source of which the output was swept from 0 to $20 \mathrm{l} / \mathrm{min}$ 13. The cardiac subsystems were each preloaded by an ideal continuous pressure source and afterloaded by their respective vascular bed model. Preload was then varied from -2 to $12 \mathrm{~mm} \mathrm{Hg}$ and the output flow and pressure recorded (Fig. 7.1).

\section{Basic model characterization}

The dynamic properties of the computer model as a whole are shown in Figure 7.1. The center curve shows the well-documented response of cardiac output to variations in the resistance to venous return ${ }^{13,20}$. The model's extrapolated response is exemplaty for a condition of increased circulatory volume (extrapolated curve, center diagram, Fig. 7.1).

The results of a steady state simulation run (without. LVAD) under baseline conditions are given in Table 7.1. Al] hemodynamic values indicate a normal physiological status 10,13,20,24. Additional baseline (no LVAD) indices derived from the primary data are: Systemic vascular resistance, SVR $=16 \mathrm{~mm} \mathrm{Hg} \mathrm{min} / \mathrm{l}$; pulmonary vascular resistance, $\mathrm{PVR}=1.6 \mathrm{~mm}$ $\mathrm{Hg} \mathrm{min} / \mathrm{l}$; right ventricular ejection fraction, $\mathrm{EFrv}=66 \%$; left ventricular ejection fraction, $\mathrm{EF} \|_{v}=63 \%$.

\section{Mirrored interventions under LVAD suppont}

For each intervention described in the experimental protocol, a simulation counterpart was produced. If necessary, model parameters were changed to mirror the experimental results qualitatively. These model manipulations were carefully documented and were limited to the following parameters: LVAD flow, circulatory volume, PVR, and intra-thoracic pressure. All other parameters were kept constant and the intrinsic behavior of the (sub-) models was preserved.

\section{Parametric analysis}

Pulmonary artery occlusions of 4 different durations $(20,40,80$, and 160 seconds) were performed to obtain occlusion-recovery characteristics. For each of the following parameters such a series was obtained for 3 different 
parameter values: LVAD flow, circulatory volume, PVR, LV contractility, RV contractility, and the distribution of compliance around the site of vessel collapse. For each individual parameter perturbation was performed either symmetrically around the baseline set-point of the model (nominal LVAD flow of $3 \mathrm{l} / \mathrm{min}$ ) or asymmetrically to address specific pathological conditions (pulmonary hypertension, hypovolemia, and right and left ventricular failure).

Table 7.1 Baseline hemodynamic indices of the computer model

\begin{tabular}{|c|c|c|c|c|c|c|c|}
\hline Venous pressure. & mean. & 5.4 & $\min \mathrm{HLg}$ & $\begin{array}{l}\text { Intra-thorncic } \\
\text { pressure }\end{array}$ & $\begin{array}{l}\text { mean } \\
+1 /=\end{array}$ & $\begin{array}{l}-4 \\
0.2\end{array}$ & 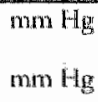 \\
\hline $\begin{array}{l}\text { Right atrial } \\
\text { pressure }\end{array}$ & mean & 2.8 & $\operatorname{mm} \mathrm{Hg}$ & Lett atrial pressuic & mean & 3.7 & mur $\mathrm{Hg}$ \\
\hline $\begin{array}{l}\text { Right venticulat } \\
\text { pressure }\end{array}$ & $\begin{array}{l}\text { sys } \\
\text { dias }\end{array}$ & $\begin{array}{l}22 \\
2.5\end{array}$ & $\begin{array}{l}\operatorname{mrn} \mathrm{Hg} \\
\mathrm{man} \mathrm{Hg}\end{array}$ & $\begin{array}{l}\text { Leti ventricular } \\
\text { pressure }\end{array}$ & $\begin{array}{l}\text { sys } \\
\text { dias }\end{array}$ & $\begin{array}{l}125 \\
2.4\end{array}$ & $\begin{array}{l}\text { mas } \mathrm{Fg} \\
\text { mom } \mathrm{Hg}\end{array}$ \\
\hline $\begin{array}{l}\text { Palmonay } \\
\text { arterial pressure }\end{array}$ & $\begin{array}{l}\text { sys } \\
\text { mean }\end{array}$ & $\begin{array}{l}21 \\
13\end{array}$ & $\begin{array}{l}\operatorname{mm} \mathrm{Hg} \\
\operatorname{mm} \mathrm{Hg}\end{array}$ & Aortic pressure & $\begin{array}{l}\text { sysi } \\
\text { mean }\end{array}$ & $\begin{array}{l}123 \\
92\end{array}$ & $\begin{array}{l}m \text { mg } \\
\text { man } H g\end{array}$ \\
\hline & dias & 10 & $\mathrm{~mm} / \mathrm{Hg}$ & & dias & 76 & $\mathrm{~mm} \mathrm{H} H$ \\
\hline $\begin{array}{l}\text { Palmonary } \\
\text { antucrial flow }\end{array}$ & $\begin{array}{l}\text { mean } \\
\text { peatin }\end{array}$ & $\begin{array}{l}5.7 \\
27\end{array}$ & $\begin{array}{l}1 / \min \\
1 / \min \end{array}$ & Aortic flow & $\begin{array}{l}\text { maean } \\
\text { peal }\end{array}$ & $\begin{array}{l}5.7 \\
37\end{array}$ & $\begin{array}{l}1 / \text { min } \\
1 / \text { mins }\end{array}$ \\
\hline $\begin{array}{l}\text { Right ventricular } \\
\text { volume }\end{array}$ & and-sys & 108 & $\mathrm{ml}$ & $\begin{array}{l}\text { Left ventricular } \\
\text { volume }\end{array}$ & $\begin{array}{l}\text { and-syss } \\
\text { and-dias }\end{array}$ & 108 & $\mathrm{ml}$ \\
\hline $\begin{array}{l}\text { Pulmonary } \\
\text { volume }\end{array}$ & & 1.7 & 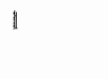 & Systemic wolume & & 40 & 1 \\
\hline
\end{tabular}




\section{RESULTS}

\section{Experimental data}

\section{LVAD support}

The hemodynamic effects of LVAD support are presented in Table 7.2. In the minimum speed mode (2200 $\mathrm{pm})$ only diastolic LV pressure ( $\mathrm{Plv}$, dias from 3.7 to $-1.6 \mathrm{~mm} \mathrm{Hg}$ ) and LV output $(-38 \%)$ were decreased significantly. These effects were increased after switching the LVAD on at $3000 \mathrm{rpm}$ : Plv, dias was $-8.3 \mathrm{~mm} \mathrm{Hg}$ and LVO decreased by $58 \%$ with respect to baseline (pump off). Additionally, tight ventricular diastolic pressure was lower than at $2200 \mathrm{rpm}(6.4$ versus $5.5 \mathrm{~mm} \mathrm{Hg}$ ) and aortic pulse pressure was significantly less $(-56 \%)$. Interestingly, the transpulmonary pressure gradient (TPG; calculated from: Ppa - Plv, dias) increased with LVAD operation: TPG $+15 \%$ and $+31 \%$ (minimum and maximum speed).

Figure 7.2 shows the relationship between the calculated pulmonary vascular resistance (PVR) and the flow through the pump (Qlvad) for each animal. Each curve shows a rightward direction with an increase in speed fiom 0 to 2200 to $3000 \mathrm{rpm}$. With LVAD support, PVR decreases in three cases (lower curves) and increases in the remainder (three upper curves). Extremely non-linear behavior is expressed by the upper curve of Figure 7.2: With the increase in speed from 2200 to $3000 \mathrm{rpm}$ PVR increases twofold and LVAD flow is actually less than at minimum speed.

For detailed evaluation of the dynamic differences between the subjects two representative cases were selected. The lower trace in Figure 7.2 (filled triangles) represents subject $A$ and the filled circles will represent subject $B$ in the following discussion. 
Table 7.2 Hemodynamic effect of LVAD support

\begin{tabular}{|c|c|c|c|c|c|c|c|}
\hline \multirow{2}{*}{$\begin{array}{l}\text { Variable } \\
\text { Plw,sys }\end{array}$} & \multirow{2}{*}{ units } & \multicolumn{2}{|c|}{ Baseline } & \multirow{2}{*}{ Mininaum $\frac{75}{75}$} & \multirow{2}{*}{$\frac{\text { speed }}{(15)}$} & \multirow{2}{*}{$\begin{array}{r}\text { Maxinum } \\
46\end{array}$} & \multirow{2}{*}{$\begin{array}{l}\text { speed } \\
(41)\end{array}$} \\
\hline & & 77 & (13) & & & & \\
\hline Plv, diass & $\mathrm{mm} \mathrm{Hg}$ & 3.7 & $(3.7)$ & -1.6 & $(9.4)$ & -8.3 & $(14)$ \\
\hline $\mathbb{P} P$ & $\mathrm{~mm} \mathrm{Hg}$ & 20 & $(3.5)$ & 15 & $(2.9)$ & 8.8 & $(31)^{\text {杜 }}$ \\
\hline Pao & $\mathrm{mm} \mathrm{Hg}$ & 62 & $(10)$ & 66 & (14) & 63 & (24) \\
\hline Pro,sys & $\mathrm{mm} \mathrm{Hg}$ & 40 & (11) & 38 & $(9.0)$ & 35 & (3.7) \\
\hline Prv, dias & $\mathrm{mm} \mathrm{Hg}$ & 4.5 & $(4.7)$ & 6.4 & $(4.2)$ & 5.5 & $(4.3)$ \\
\hline Ppa & $\operatorname{mm} \mathrm{Hg}$ & 29 & $(10)$ & 29 & $(10)$ & 25 & $(5.1)$ \\
\hline Qpa & $1 / \mathrm{min}$ & 4.5 & (1.3) & 5.4 & $(1.9)$ & 5.2 & $(2.4)$ \\
\hline Qlvad & $1 / \mathrm{min}$ & $0^{*}$ & & 2.6 & $(0.6)^{\text {新 }}$ & 3.3 & $(1.0)^{3+i}$ \\
\hline LVO & $1 / \min$ & 4.5 & (1.3) & 2.8 & $(1.9)^{3}$ & 1.9 & $(1.5)^{4 *}$ \\
\hline TPG & $\mathrm{mm} \mathbb{H g}$ & 26 & $(12)$ & 30 & $(13)$ & 34 & (17) 非 \\
\hline PVR & WU & 6.8 & (5.8) & 6.9 & $(5.9)$ & 10 & $(12)$ \\
\hline
\end{tabular}

Mean (SD), n=6. LVAD, left ventricular assist device; Maximum/minimum speed: $3000 / 2200 \mathrm{rpm}$. Plv,sys, left ventricular systolic pressure; Ply, dias, left ventricular diastolic pressure; PP, pulse pressure (maximum - minimum aortic pressure); Pao, mean aoticic pressure; Prv,sys, right ventricular systolic pressure; Prv,dias, right ventricular diastolic pressure; Ppa, mean pulnonary atterial pressure; Qpa, mean pulmonary artetial flow; Qlvad, mean LVAD flow; LVO, calculated left ventricular output (Qpa - Qlvad); TPG, transpulmonary pressure gradient (Ppa - Plv,dias); PVR, pulmonary vascular resistance (TPG/Qpa); WU, Wood units. \&VAD outflow graft occluded (no aortic regurgitation). Wilcoxon signed-ranks test. ${ }^{*} p<.05$ vs. baseline; ${ }^{t} \mathrm{p}<.05$ vs. minimum speed. 


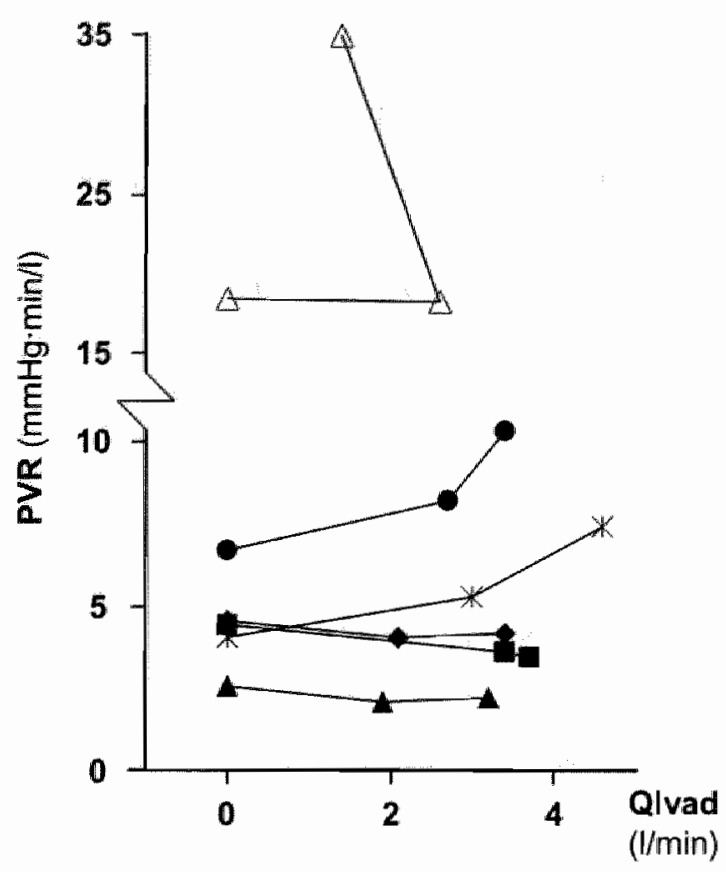

Figure 7.2 Individual relationships between pulmonary vascular resistance (PVR) and mean LVAD flow (Qlvad) as a result of LVAD operation at no LVAD flow and minimum and maximum speed $(n=6$ ). (PVR is calculated from: (mean pulmonary artery pressure minur diastolic left ventricular pressure) divided by mean pulmonary artery flow.)

\section{Pulnonary artery octusions}

In general, PAOC produced a marked increase in RV systolic pressure and strong decrease of pressures downstream the occlusion site. Without LVAD operation aortic pressure and systolic LV pressure jointly decreased, whereas with the LVAD on aortic pressure increased above LV pressure which became negative (suction). Typically, after the release of the occlusion subsequent hemodynamic recovery would occur within about the duration of the occlusion. 
We will now provide a more detailed presentation of the PAOC results based on Figures 7.3 and 7.4. The trends were obtained from subjects $A$ and $B$ defined above. During PAOC ventilation settings were kept constant.

Figure 7.3 shows a substantial rise in RV afterload $(+100 \%)$ and an acute cessation of pulmonary arterial flow upon occlusion. Due to the fact that the pulmonary arterial pressure sensor lay distal of the occlusion tourniquet, the Ppa trace indicates pulmonary wedge pressure during the occlusion. The obvious cut-off in the upstream flow generates the anticipated decay of arterial and ventricular pressures during the first 10 seconds of the occlusion. The resultant negative values of Plv (and Pla initially) indicate the suction due to continuous LVAD aspiration although

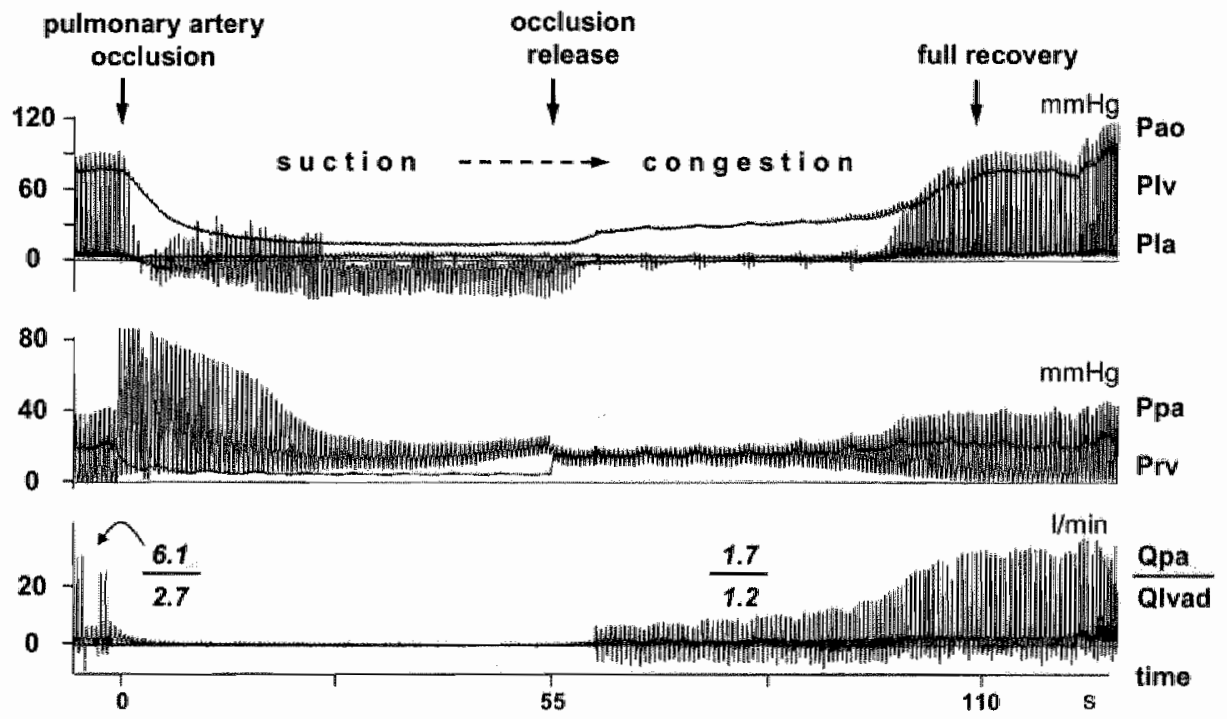

Figure 7.3 Pulmonary artery occlusion during LVAD speed $3000 \mathrm{rpm}$. Data from subject A. Pao, aortic pressure; Plv, left ventricular pressure; $P l_{2}$, left atrial pressure; Ppa, pulmonary arterial pressure; Prv, right ventricular pressure; Qpa, pulmonary arterial flow; Qlvad, LVAD flow. The occlusion during 55 seconds produces severe suction already after 5 heart beats. Upon release hemodynamic recovery remains incomplete for 30 seconds (congestion). Full hemodynamic recovery occurs spontaneously within 55 seconds after release of occlusion. In the lower graph the average pre occlusion values of Qpa and Qlvad and during congestion are printed. 
the net pump flow is attenuated. A steady mean pressure gradient of about $15 \mathrm{~mm}$ Hg between Pla and Plv is noted. Immediately before release of the toumiquet a strongly reduced RV contractility and systemic venous congestion ate apparent. After 55 seconds the release is initiated and pulmonary flow slowly regains its impetus. However, signs of congestion persist for another 30 seconds. Ultimately, full hemodynamic recovery rapidly ensues from the point where Plv touches Pla (Fig. 7.3).

In subject $B$ we obtained the trend depicted in Figure 7.4. Qualitatively, the response to the 20 second PAOC is comparable to that presented in Figure 7.3. However, hemodynamic recovery did not occur
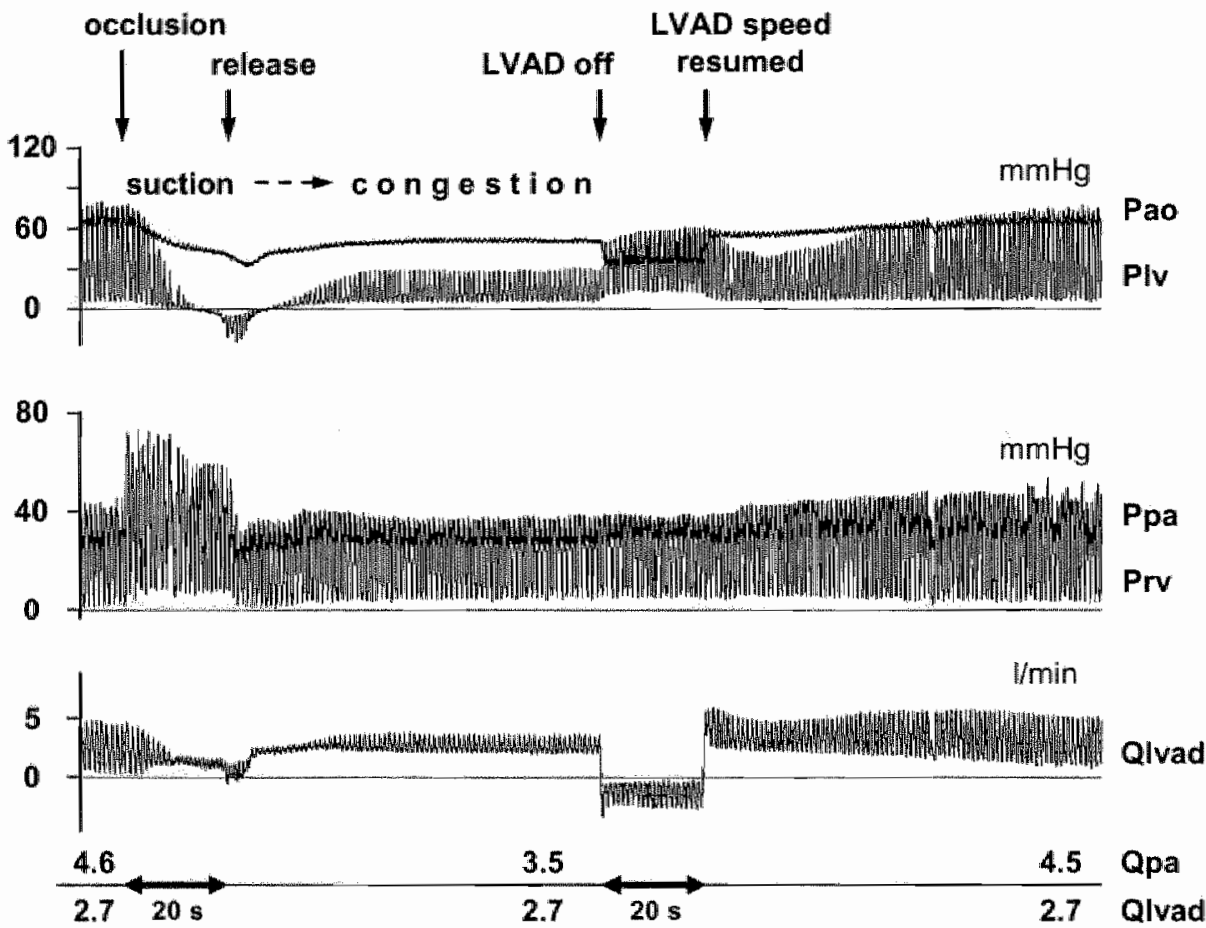

Figure 7.4 Pulmomary artery occlusion during LVAD support with $2200 \mathrm{upm}$ in subject B. Pao, aortic pressure; Plv, left ventricular pressure; Ppa, pulmonary arterial pressure; Prv, right wentricular pressure; Qlvad, LVAD flow; Qpa, pulmonary arterial flow (trace is omitred for the sake of brevity). The occlusion duration is 20 seconds. The Ppa sensor lies upstream of the occlusion site. Halfway the time line the LVAD speed is reduced to $0 \mathrm{tpm}$ for a period of 20 s (functional aorric insufficiency), after which operation is continued at the pre occlusion speed (2200 rpm). 
within the anticipated period, which prompted the intuitive intervention of turning the LVAD off for a brief period. 'This 'pump down' intervention without occluding the outflow graft produced aortic insufficiency as can be appreciated from the Qlvad trace in Figure 7.4. Subsequently, full recovery followed 'pump down' after a delay of 20 seconds. Any notion of a depressed RV function was absent as opposed to the previous case. In subject $B$ left atrial pressure measurements were not obtained.

\section{Pump speed manipulation}

The trend shown in Figure 7.5 was obtained in subject B after transiently switching the LVAD from minimum to maximum speed. Qlvad increased from 2.7 to $3.71 / \mathrm{min}$ and Plv decreased due to the augmentation in

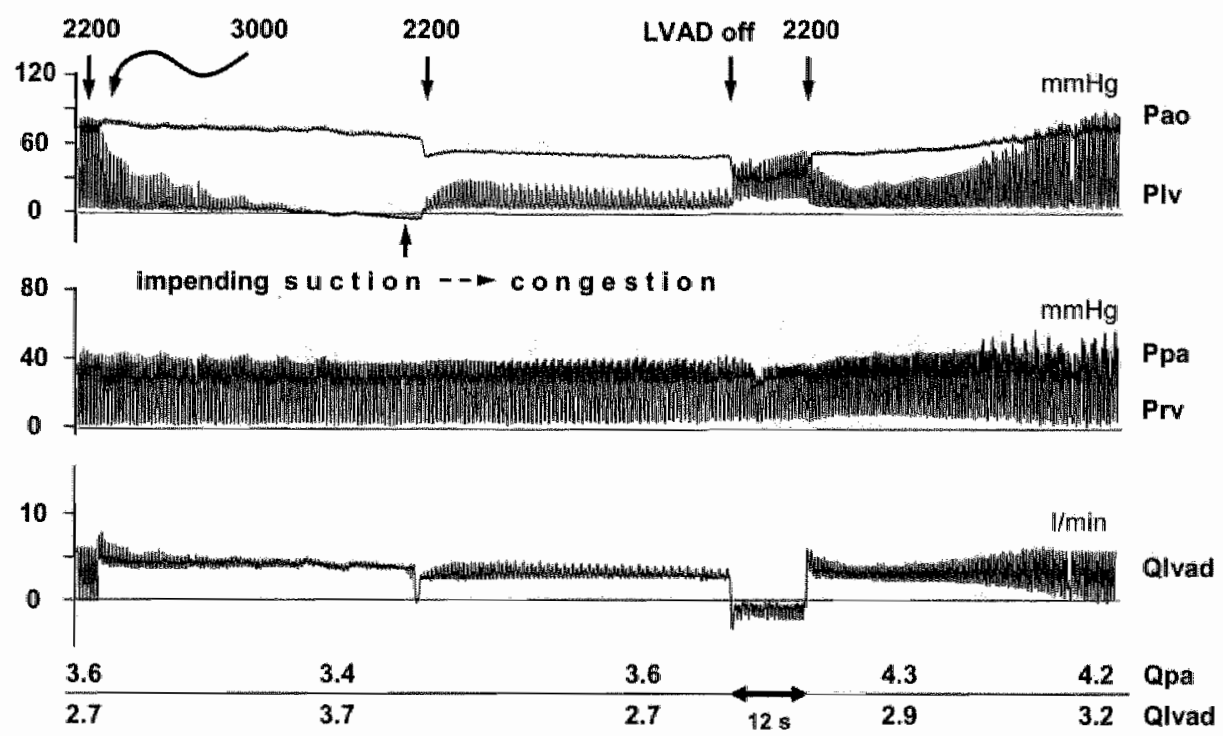

Figure 7.5 (Subject B) Hemodynamic tesponse after switching the LVAD speed from 2200 (Qlvad $2.7 \mathrm{l} / \mathrm{min}$ ) to $3000 \mathrm{~mm}$ (Qlvad $3.7 \mathrm{l} / \mathrm{min}$ ). Pao, aortic pressure; Plv, left ventricular pressure; Ppa, pulmonary arterial pressure; Prv, right ventricular pressure; Qlvad, LVAD flow; Qpa, pulmonary arterial flow. In maximum speed mode a gradual decrease in Pao, Plv, and Qpa is noted. To prevent the (impending) suction, the LVAD is switched back to minimum speed after 55 seconds. Return to the initial profile does not occur and Pao remains depressed. After another 55 seconds a 'pump down' intervention of 12 s duration is initiated. 
unloading (No forward fiow across the aortic valve). Although aortic pressure remained fairly stable, Plv showed impending suction (negative LV pressures). To counter the further progtess towards full suction the speed was then reduced to the initial level $(2200 \mathrm{rpm})$. Both Qlvad and Qpa retumed to their initial values, but the depression in Pao persisted. Electively, a 'pump down' intervention was performed after which the hemodynamic variables gradually retained their initial values. The episode ended in a compensatory state with increased flows and ventricular pressures (Fig. 7.5).

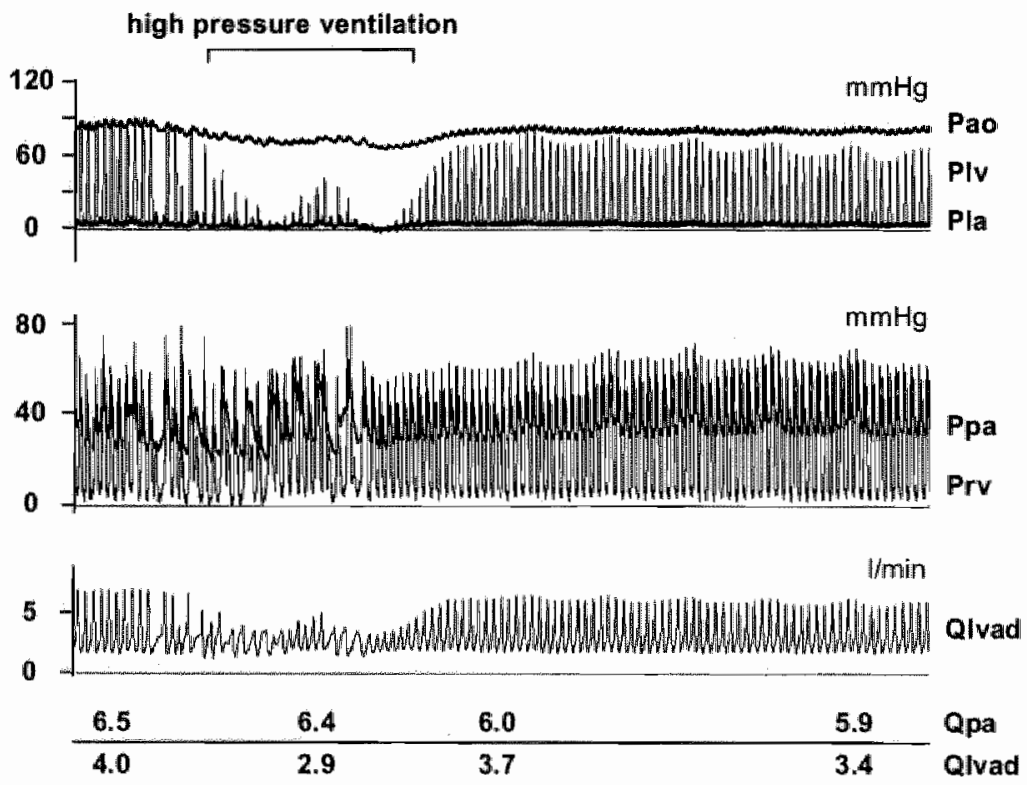

Figure 7.6 (Subject A) Hemodynatnic response to episodic manual ventilation. (Before and after the intervention identical ventilation settings are maintained.) $\mathrm{PaO}_{\mathrm{a}}$ nortic pressure; $\mathrm{Plv}$, left ventricular pressure; $\mathrm{Pla}$, left atrial pressure; $\mathrm{P}$ pa, pulmonary arterial pressure; Prv, right ventricular pressure; Qlvad, LVAD flow; Qpa, pulmonary arterial flow (Trace is omitted for the sake of brevity). 


\section{Manual ventilation}

Subject $A$ was subjected to an episode of manual ventilation during LVAD operation at $3000 \mathrm{rpm}$. Upon inspection of Figure 7.6 a post-interventional progressive decrease in Qpa, Qlvad, and systolic Plv was noted.

\section{Simulation results}

\section{Pulmonary artery occlusions}

Occlusion of the pulmonary artery was simulated by temporarily increasing the lumped resistance in the proximal section of the pulmonary vascular bed by a factor of 10 .

The pre-occlusion hemodynamic profile in Figure 7.7 is different from the model's baseline values given in Table 7.1. First of all the model included the LVAD set to deliver around $31 / \mathrm{min}$ under baseline conditions. Intra-thoracic pressure was increased from -4 to $-1 \mathrm{~mm} \mathrm{Hg}$ to mock the open-thorax setting in vivo. Furthermore, to obtain a faithful simulation of the experimental counterpart PVR was increased from 1.8 to 4.9 (mm $\mathrm{Hg} \mathrm{min}) / 1$ (or Wood units, WU) and circulatory volume was increased from 5.7 to 6.2 liters (i.e. $+500 \mathrm{ml}$ ). These adjustments created a pre- occlusion condition that featured elevated PVR and increased filling (to compensate for the loss in cardiac output and aortic pressure), which matched Figure 7.3 best.

Qualitatively, the dynamics of the 55 second PAOC resemble those presented in Figure 7.3, namely: Acute RV afterload elevation, downstream decay of pressures, initial negative Plv and Pla values and the subsequent gradient between these, and delayed spontaneous recovery. Second-order effects on RV contractility, aortic pressure, and pump flow however are absent (refer to Fig. 7.3). Qlvad, Qpa, and Pao do decrease during occlusion but are not as depressed as seen in vivo. 

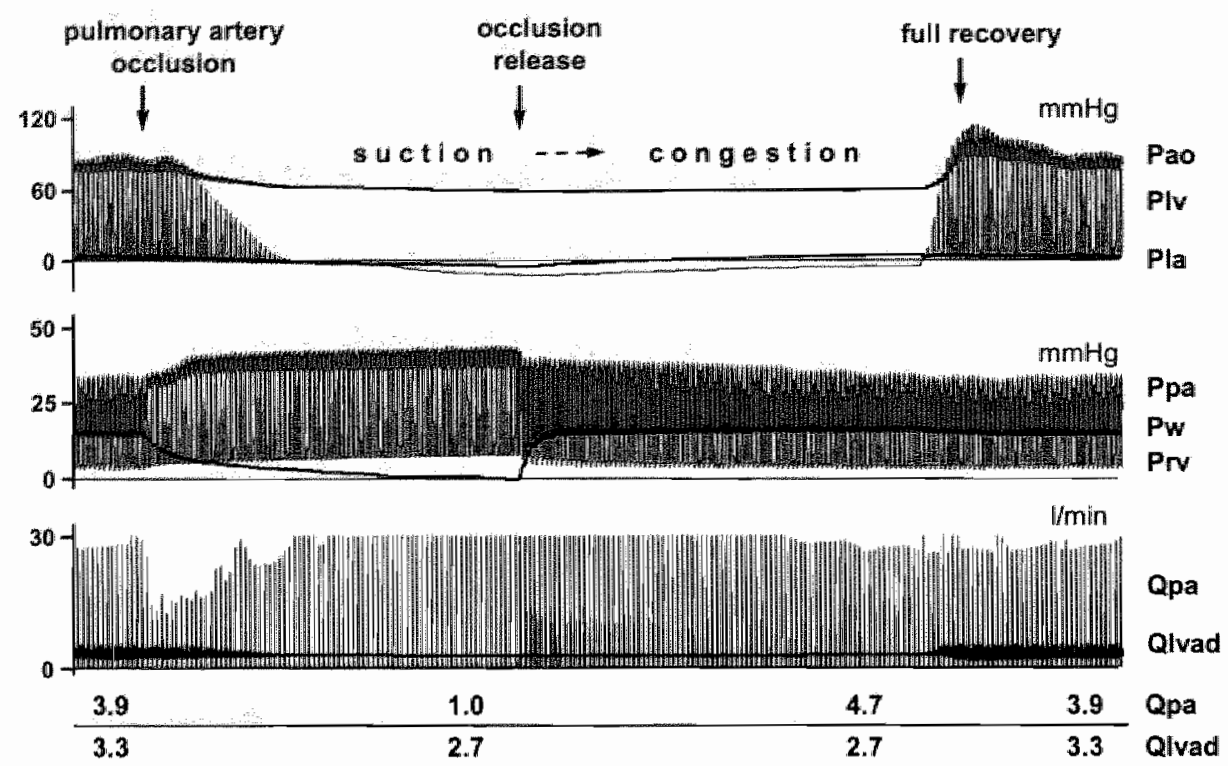

Figure 7.7 Simulated 55 second pulmonary artery occlusion. Pao, aortic pressure; Plv, left ventricular pressure; $\mathrm{Pla}$, left atrial pressure; $\mathrm{P}$ pa, pulmonary arterial pressure; $\mathrm{Pw}_{\mathrm{w}}$ pulmonary wedge pressure; Prv, right ventricular pressure; Qpa, pulmonary arterial flow; Qlvad, LVAD flow.

With the simulation run shown in Figure 7.8 the pre-occlusion PVR elevation was maintained. However, circulatory filling was left untouched as aortic pressure and cardiac output in vivo (Fig. 7.4) were less than in the previous case. When we compare the pre-occlusion conditions in Figures 7.7 and 7.8 , an increase in the level of LV bypass (100\% Qlvad/Qpa) from $85 \%$ to $97 \%$ is noted.

Again first-order dynamics appear to comply with the experimental case. However, in this simulation the LV remains empty after release of the occlusion. Furthermore, a 'pump down' intervention leads to hemodynamic recovery more rapidly than in vivo. A meta-simulation without pump intervention showed the congestion would have persisted at least 15 minutes. 

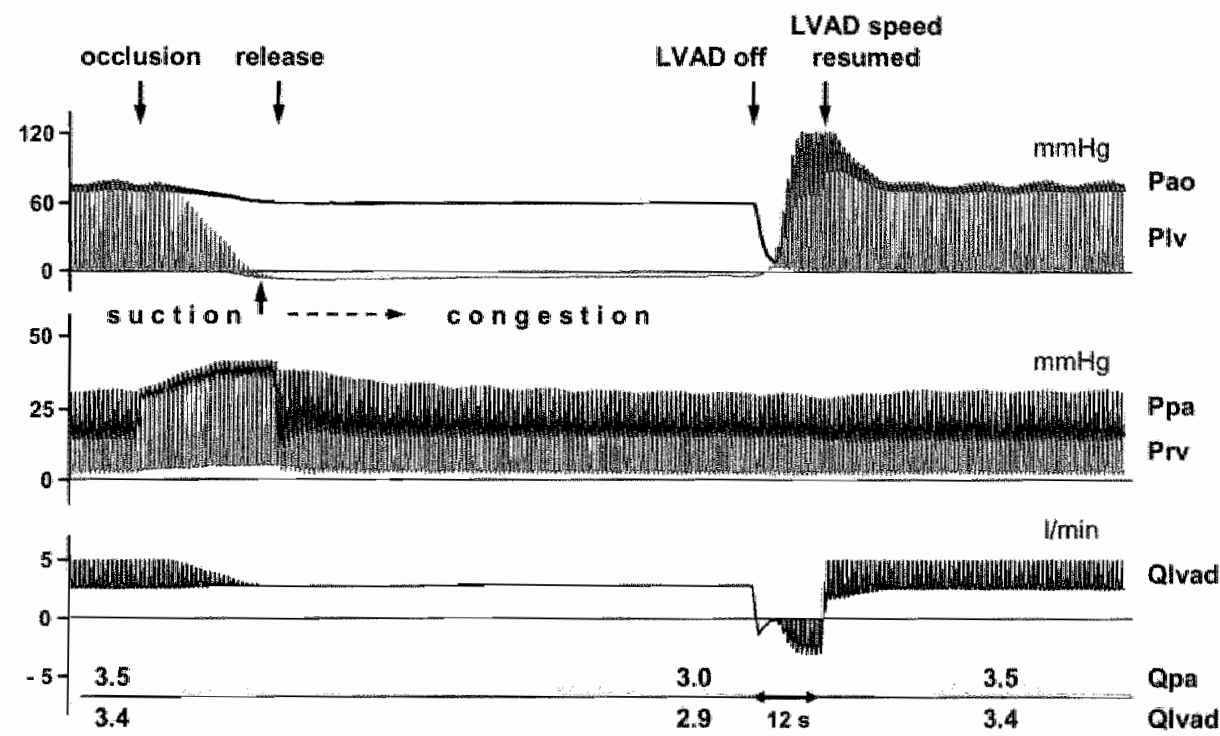

Figure 7.8 Pulmonary artery occlusion with no spontaneous recovery. Pao, aortic pressure; Plv left ventricular pressure; $\mathbb{P}$ pa, pulmonary arterial pressure; Prv, right ventricular pressure; Qpa, pulmonary arterial flow; Qlvad, LVAD flow. 


\section{Simulated speed manipulation}

The speed manipulation simulation (Fig. 7.9) was based on exactly the same initial conditions as with the PAOC, i.e. elevated PVR, opened thoracic cavity, and a LVAD set to $-31 / \mathrm{min}$. Upon inspection of Figures 7.5 and 7.9 the behavior of the pump flow (Qlvad) are similar and the general experimental and simulated dynamics appear congruent qualitatively. The pump intervention necessary to facilitate full hemodynamic recovery took 7 seconds. The meta-simulation without pump intervention showed the congestion would have persisted at least 15 minutes.

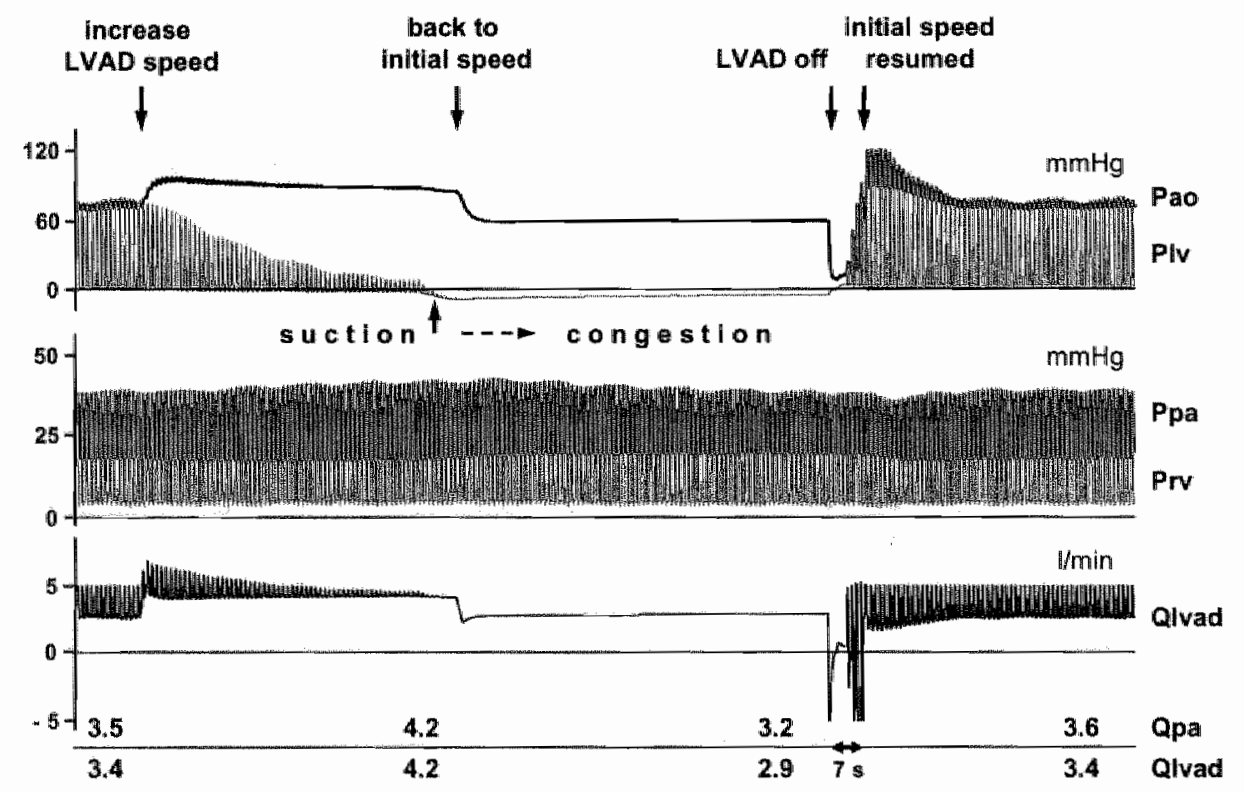

Figure 7.9 LVAD up-switch from 3.4 to $4.21 / \mathrm{min}$ in simulation. The pump flow setting is reversed as soon as suction becomes apparent. Subsequently, signs of congestion persist and a 'pump down' intervention is performed. $\mathrm{PaO}$, aortic pressure; Plv, left ventricular pressure; Ppa, pulmonaty arterial pressure; Prv, right ventricular pressure; Qpa, pulmonary arterial flow; Qlvad, LVAD flow. 


\section{Intra-thoracic preswe disturbance}

Three distinct modes of intra-thoracic pressure (Pit) disturbance were simulated: (1) sudden elevation of Pit from -1 to $+3 \mathrm{~mm} \mathrm{Hg}$ during 10 seconds involving all intra-thoracic vascular compliances, (2) an elevation as in (1) involving only the proximal pulmonary venous compliance, and (3) a drop in Pit from -1 to $-4 \mathrm{~mm} \mathrm{Hg}$ again only involving the proximal pulmonary venous compliance. All three simulations resulted in signs of congestion after the intervention. Figure 7.10 shows the trend following the third mode intervention. This trend was selected because it fit its experimental counterpart (Fig. 7.6) best qualitatively considering Pao and Plv. Filling of the LV after the intervention on Pit is marginal and forward flow runs only through the LVAD, i.e. the LV fully bypassed. The metasimulation again predicted an extended ( $>15$ minutes) recovery delay, therefore a sudden decrease in LVAD flow was initiated after which recovery rapidly evolved (Fig. 7.10).
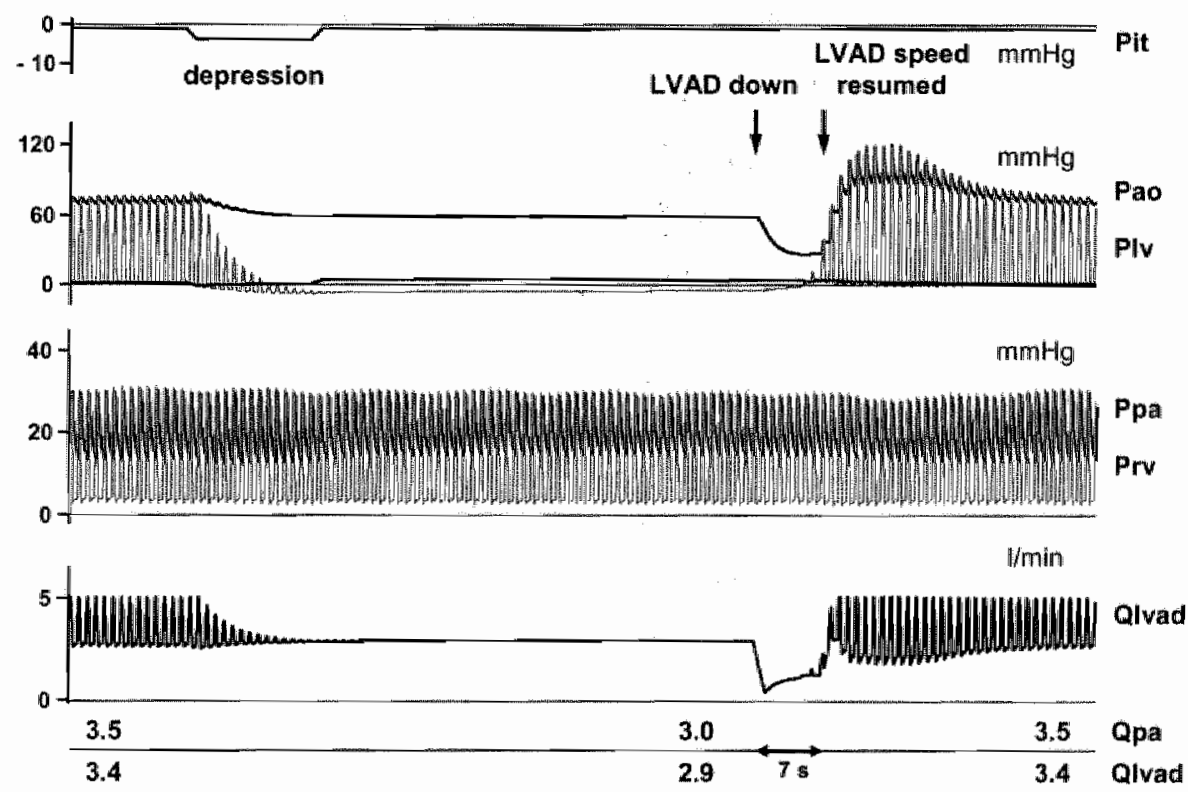

Figure 7.10 Respiratory artifact imposed on the post-capillary section of the pulmonary vascular bed (computer simulation). Pit, intra-thoracic pressure; Pao, aortic pressure; Plv, left ventricular pressure; Ppa , pulmonary arterial pressure; Prv, right ventricular pressure; Qpa, pulmonary arterial flow; Qlvad, LVAD flow. 


\section{Parametric analysis}

The PAOC of various durations produced recovery delays ranging from half the occlusion period (typically 8 seconds) to beyond 100 times the occlusion time. The occlusion-recovery relationship was dependent on the six evaluated parameters, namely: LVAD flow, circulatory volume, PVR, RV contractility, LV contractility, and the distribution of compliance around the site of vessel collapse. Evaluation of the last two parameters showed only marginal impact on the dynamics of suction as induced by PAOC. Compared to the nominal curve (contractility $100 \%$ ) a LV contractility of $25 \%$ increased recovery time with a mere 10 seconds. A similar change was produced by (any) redistribution of the lumped compliance values in the pulmonary venous section. Hence, no diagrammatic presentation is provided for these parameters. The characteristics of the remaining four are given in Figure 7.11.

In all four panels, the thick traces represent the simulation characteristic under nominal conditions. The parameter curves show exponential behavior: E.g. at a low LV bypass flow $(1.2 \mathrm{l} / \mathrm{min})$ hemodynamic recovery takes less than 20 seconds after periods of suction up to 160 seconds. However, with increasing flow settings tecovery delays increase disproportionately (Fig. 7.11, upper left panel). Reductions in circulatory volume and RV contractility, as well as increases in PVR increase the time necessary to overcome the signs of congestion (refer to Figs. 7.7 and 7.8). 


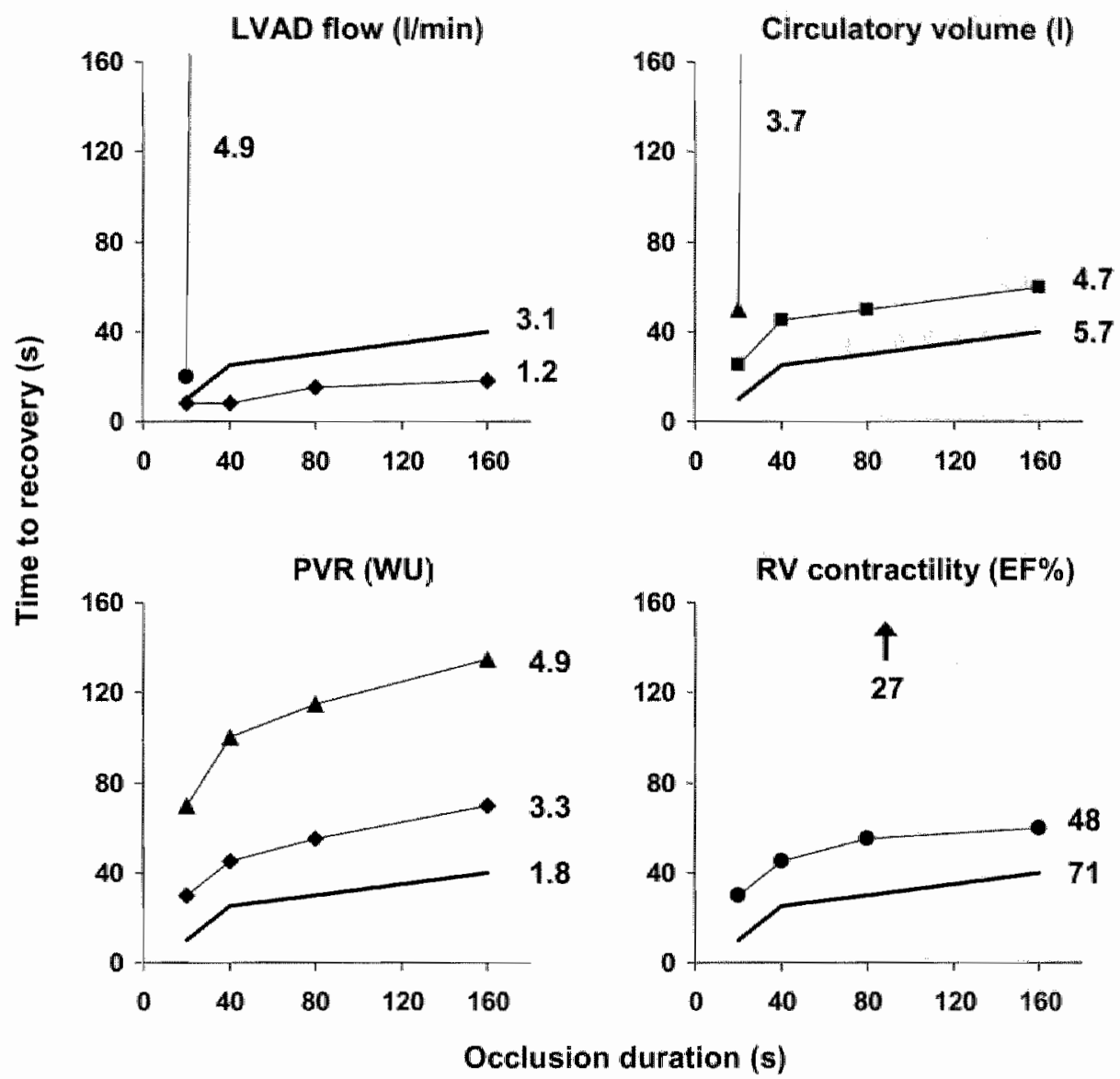

Figure 7.11 Dependency of the recovery time after pulmonary artery occlusion in the face of (singular) parameter variations. The release of the occlusion is defined as zero time to recover. PVR, pulmonary vascular resistance (in Wood units); RV, right ventricular; EFo, ejection fraction in percent. The simularion series with the nominal parameter values are depicted by the thick curres. The times-to-recover of the data points lying out of range in the upper wwo diagrams all exceed 2000 seconds. Curve for RV contractility (EF) of $27 \%$ lies entirely above 1000 seconds. 


\section{DISCUSSION}

\section{LVAD support in vivo}

In all six animals LVAD support decreased left ventricular preload, stroke volume, and afterload. With support at maximum speed decreased loading of the right ventricle was noted (Table 7.2), but due to the limited number of subjects only RV preload decrease achieved significance. Interestingly, the pressure gradient across the pulmonary bed increased by $30 \%$, and the calculated pulmonary vascular resistance (PVR) increased from 6.8 to 10 Wood units. From clinical experience it is, however, known that LVAD support unloads the right side and may thereby improve pulmonary hypertension and decrease PVR ${ }^{1-3}$. The origin of this inconsistency becomes clear upon examination of Figure 7.2. In three animals the response of PVR to LVAD support complies with the anticipated decrease in pulmonary circulatory loading, but in the remaining three (upper curves) the effect of support seems counterproductive. The latter is well illustrated by the upper curve which shows the LVAD actually overloads the right side. The LVAD flow-PVR analysis provides at least one clue to what causes the differences in dynamic: Pre-support PVR is correlated to the subsequent increase of PVR when the LVAD is switched on. The fact that elevated PVR and pulmonary hypertension predispose right-sided circulatory failure with LVAD recipients has been addressed in both clinical and experimental contexts ${ }^{9-11}$.

In order to explain the non-linear behavior identified in the flowresistance analysis we will now focus on the results obtained with pulmonary artery occlusions in vivo and by simulation.

\section{Determinants of suction}

Upstream blockage of flow proved to be an effective and reproducible means to elicit suction at the pump inlet (Figs. 7.3, 7.4, 7.7, and 7.8). During PAOC the continuous aspiration of the LVAD created negative left atrial and ventricular pressures in the early stage of suction and a stable pressure 
gradient of 10-20 $\mathrm{mm} \mathrm{Hg}$ between atrium and ventricle (Figs. 7.3 and 7.7). In these cases vessel collapse occurs in between the left atrium and the pump inlet, which decreases the forward flow but also limits the further decline of inlet pressure. Because the collapsed segment itself is held closed by the pump's continuous aspiration, the suction condition is self-stabilizing during PAOC. When the occlusion ends, however, resolution of the collapse does not immediately take place and a period of persistent congestion may follow (Figs. 7.3 and 7.7). Both the experimental and simulated trends show a slow time-dependent regression of the left ventricular pressure towards the left atrial pressure upon which hemodynamic recovery ensues. As the examples in Figures 7.4 and 7.8 show, the signs of congestion may last for an extended period, which could prove fatal in clinical situations. Only a slight shift in circulatory volume (in Fig. $7.8500 \mathrm{ml}$ less than in Fig. 7.7) produced congestion for more than 15 minutes during simulation. In addition to hypovolemia, PVR elevation was a concomitant factor for the extension of the recovery delay.

The parameter evaluations (Fig. 7.11) identified four main determinants of suction and their effect on the pulmonary circulatory function after an occlusive event upstream. From clinical experience it is argued that during full LV bypass, suction will occur more likely than with partial support ${ }^{12,16-18,26-29}$. The results of this study confirm this but also imply that the effectiveness of LV bypass strongly depends on the patency of the pulmonary circulation. Concomitant right ventricular failure and pulmonary hypertension are known complications of LVAD therapy $1,2,10$. Under pathophysiological conditions LV bypass ratios of even less than $50 \%$ may lead to suction problems when venous return is disturbed (Figs. 7.3 and 7.4 ).

So far, we have considered intentionally elicited suction by which we obtained results that are consistent with peer clinical and experimental research. We will now discuss the results obtained by the more realistic interventions on LVAD flow and extravascular pressure. 


\section{Suction induced collapse}

Sudden increases in pumping speed are associated with brief (oscillatory) episodes of suction ${ }^{1726,27}$. Either avoidance of transient manipulation or trial and error adjustment (Figs. 7.5 and 7.9) are usually regarded sufficient to avoid suction problems. However, the latter approach proves insufficient in Figure 7.5: Full-blown suction is avoided by the speed readjustment $(3000$ back to $2200 \mathrm{rpm}$ ), but signs of congestion ensue and appear to persist. When we compare the trend in vivo with the simulation (Fig. 7.9) we may conclude that the overall response is similar and attributable to vessel collapse. However, whereas the conditions for collapse are clear in simulation (as it is specifically designed), the gravity and location of collapse are less evident in vivo. Only from the notion of congestion can we derive that the upstream path of flow was altered. Because right ventricular loading and output apparently have not changed, we conclude that vascular resistance has increased. Additionally, left ventricular pressure is positive during the congestive period in vivo (Fig. 7.5), so the collapsed section can not be localized between atrium and pump inflow. Furthermore, the obstructive effect appears less severe compared with the computer model (Fig. 7.9). Thus, we may infer that aspiration induced congestion entails not only the obvious occlusive on-off dynamic.

Because pulmonary vascular resistance is by no means a straight forwatd concept ${ }^{20,24,30}$, discrimination of the partial resistances involved is mandatory. For the pulmonary vascular bed we assume the statling resistor/distension/recruitment concept to be appropriate ${ }^{13,21 \cdot 23,30}$. For the purpose of analysis the location of the collapsible vessel is assumed to lie somewhere in between the pulmonary venular region and the LVAD inlet. Figure 7.12 presents the implied configuration of a pulmonary starling resistor in series with a suction induced starling resistor (SISR). The pulmonary resistor sees the pressure upstream the SISR as its back pressure (Pim), while the pump inlet pressure (Plv) constitutes the back pressure for the SISR. The intra-thoracic pressure (Pit) is the effective extramural pressure of the SISR. It is obvious that if Plv becomes smaller than Pit the SISR closes, the flow through the pulmonary resistor ceases, and the 
pulmonary back pressure Pin becomes elevated. If we assume that the pulmonary resistor was initially operating in West zone II, SISR closure will induce a transition to West zone III ${ }^{23,30}$. This causal relationship remains tenable even when the SISR is not completely closed. In abnormal physiological situations the zonal conditions of the pulmonary bed remain dependent on the effective back pressure ${ }^{21 \cdot 24,30,31}$. The trend in Figure 7.5 illustrates that with partial collapse pulmonary hemodynamics may seem unaltered, presumably due to adaptations in PVR, but nonetheless right ventricular loading (Prv, dias up) is increased and LV/LVAD output reduced.

\section{Pulmonary circulatory "latch up"}

Analysis of the pump flow manipulation (Figs. 7.5 and 7.9) identified different hemodynamic profiles before and after flow increase. This implies that two distinct steady states may exist with equal values for the mean LVAD flow. Firstly, the transition seems to be brought about by the rise in pump flow and the flow readjustment made in anticipation of suction.

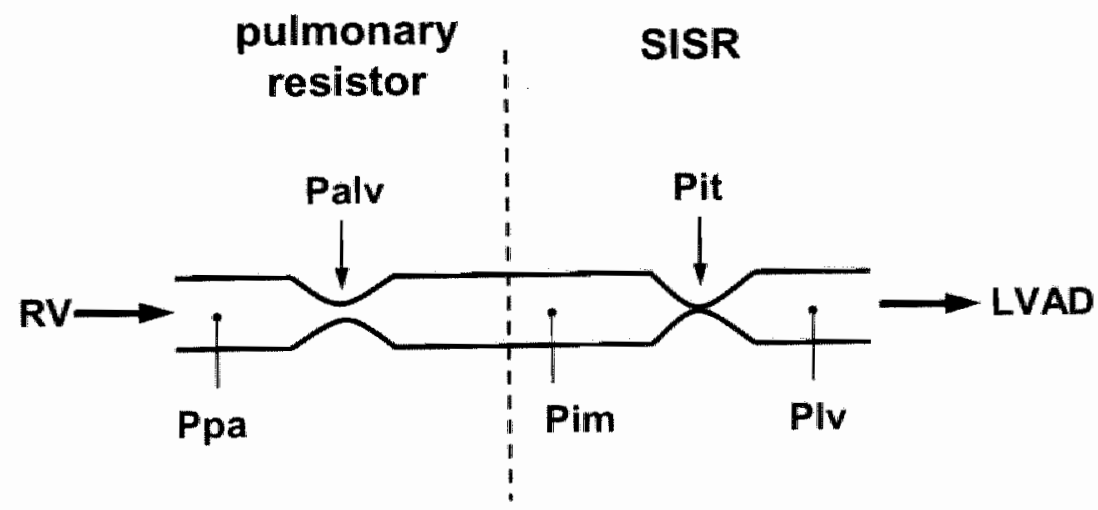

Figure 7.12 Coneept of a series connected pulmonary starling resistor and suction induced starling resistor (SISR); RV, right wentricle; Ppa, pulmonary artery pressute; Palw, alveolar pressure; Pim, intermediate pressure; Pit, intrathoracic pressure; $\mathrm{Pl}$, left ventricular pressure. 
Secondly, the models responded similarly to a temporary shut down of the LVAD which resulted in hemodynamic recovery. Apparently, acute manipulations of LVAD flow are capable of producing different functional states of the pulmonary circulation similar to the flip of a switch. This transitory behavior is summarized graphically in Figure 7.13.

The exposition in the input-output plane indicates hysteresis in the pressure-flow relationship. When we consider the direction in which the loop is exercised, it is evident that from state $A^{\prime}$ the way to recovery is via a pump flow decrease (via state $\mathrm{C}$ ). In these measurements the magnitude of the decrease in LVAD flow proved sufficient but should not be considered indicative necessarily, because the effect also depends on duration. The

\section{EXPERIMENT}

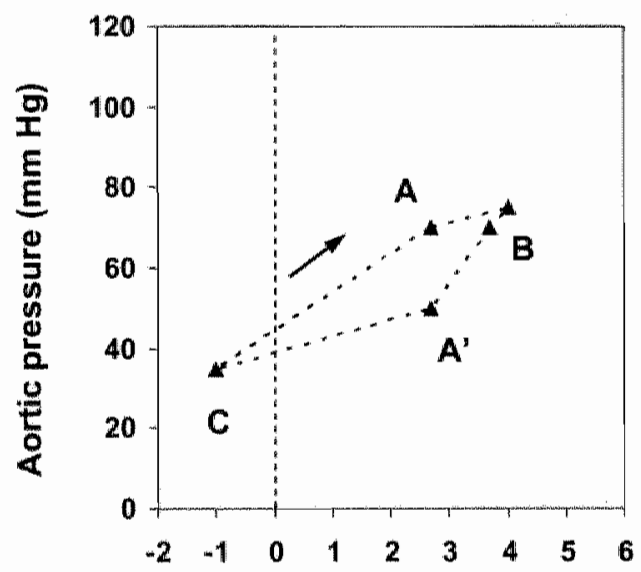

SIMULATION

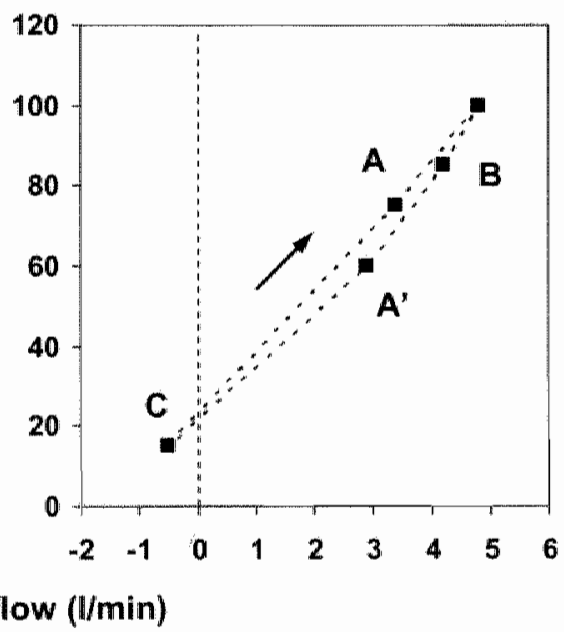

Figure 7.13 Relationship between LVAD flow and aortic pressure in both models. A: Stable partial assist. From point $A$ up and rightward the LVAD flow is increased transiently. B: Unstable situation (after initial rise in pressure) with increased LVAD flow progressing towards suction at the inlet. From point $B$ to $A^{\prime}$ the LVAD is intentionally set to the previous value (as in $A$ ) to prevent suction. A': Stable condition with depressed aortic pressure. From $A^{\prime}$ to $C$ the LVAD is switched off and aortic insufficiency ensues for a short period (LVAD flow is negative). Upon resumption of LVAD flow (as in A and $A^{\prime}$ ) point $A$ is reached again. 
common sense response to the depression in aortic pressure (Fig. $7.13 ; \mathrm{A}^{\prime}$ ), that is to retum to state A via B by increasing LVAD speed, will only elicit suction promptly with obvious results (refer to Figs. 7.3, 7.4, 7.7, and 7.8) ${ }^{32}$. Apparently, over-aspiration, imminent suction, and full suction can elicit "latch up" of the pulmonary vascular bed which may only be reversed by discontinuing pump aspiration.

\section{Extravascular pressure}

Our interventions on extravascular pressure resulted in the trends presented in Figures 7.6 and 7.10. It should be noted that in the computer simulation all conceivable extravascular pressures were represented by the intrathoracic pressure source $\mathrm{Pit}^{25}$. In vivo, alveolar pressure (as determined by mechanical ventilation) and intra-thoracic pressure (due to the opened thorax setting) constituted the effective surrounding pressure for the vessels.

After periodic manual pressure ventilation, progressively unstable ventricular unloading was noted (Fig. 7.6), comparable to the congestive episode in Figure 7.5. Unfortunately, the length of this measurement was restricted by the pilot protocol. The dynamics of the simulation comply with the basic trend in vivo, but appear exaggerated. Both responses sustain the mechanistic interpretation of "latch up" on the basis of a suction induced starling resistance (tefer to Figs. 7.12 and 7.13). Lastly, the simulated pump down intervention after respiratory artifact shows that full shut down of flow is not required to return to the initial hemodynamic state, as pointed out earlier.

Above, all mechanistic aspects of continuous aspiration induced collapse have been discussed. The implications for clinical practice and automatic feedback control design will be treated next.

\section{Clinical implications}

Recently, two studies were published on the clinical use of LVADs aimed at left ventricular unloading and normalization of pulmonary vascular 
resistance. Klotz et al, found comparable clinical results for patients supported with a non pulsatile LVAD $(n=10)$ and a pulsatile VAD $(n=21)$. However, their time regression analysis showed a significant decline in $\mathrm{LV}$ unloading efficacy in the non-pulsatile group between post-implant days 60 and 120 , which could not be explained. A group of six patients was studied by Martin et al: Five pulsatile device recipients and one continuous flow case ${ }^{3}$. Even with maximum speed support this last patient remained in NYHA class III and IV and showed insufficient progress in PVR normalization. The lack of output obtainable with the device was considered as the prime limitation and the patient was switched to a pulsatile device. The present study shows that the unloading efficacy by continuous aspiration depends strongly on the patency of the backward flow path. Moreover, the results indicate that aspiration itself may trigger unfavorable conditions for the pulmonary circulation as a result of speed maladjustment or thoracic disturbance (Figs. 7.5, 7.6, and 7.13). The effects of over-aspiration (Fig. 7.12) might explain the suboptimal results obtained with continuous flow LVADs in the studies mentioned above. The effect of an aspiration induced extra resistance on the development of cardiopulmonary (vascular) remodeling, may be comparable to that induced by left heart failure and congenital obstruction of pulmonary venous return $10,20,24,31,33$. Experimental studies on the physiological impact of abnormal pulmonary venous return can be found in the articles by Mitzner, Ducas, and Fang ${ }^{21-23}$.

Interestingly, the more prudent approach followed by the group of Salzberg, which appears particularly in line with the results of this paper, produced good results in six patients supported by a continuous flow device$^{4}$. Their protocol included controlled volume management and pharmacological support, low to moderate LVAD speeds in the early postoperative phase, and a subsequent increase in support when permitted by the patient's overall condition. To link this rationale to the results from this study, one may interpret their protocol as: First clear off any predisposing factors for suboptimal unloading and thereby shift the limitations (refer to Figs. 7.2 and 7.11), and then maximize support. 
From this we conclude that the setup and management of LVAD therapy should take in account the spurious effects and limitations of continuous flow (i.e. load insensitive) assist devices in view of the multifactorial and dynamic nature of the surrounding patho-physiological processes ${ }^{4,34}$. In clinical practice, suction or near suction may be induced by events such as acute pulmonary embolism, right coronary embolism, airway obstruction, and positive pressure ventilation.

Besides the limitations it poses, the observed non-linear behavior with pump speed manipulations as such could be put to use in the setup stage of LVAD support. The controlled envitonments of the operating room and intensive care unit permit a safe exploration of the limits on LVAD flow ${ }^{34}$. An intervention like in Figures 7.5, 7.9, and 7.13 can be employed to assess the dynamic response of the assisted circulation and thus may be of aid in diagnosing underlying tight-sided circulatory failure.

\section{Automatic feedback control}

During sustained LVAD support (patient on ICU, ambulatory, or in outpatient program) the therapeutic environment is distinctly different from the initial phase. Acute events are potentially more hazardous because timely specialist intervention may be unavailable. Furthermore, the systemic condition of the LVAD recipient changes with time owing to support itself (recovery, overload), as well as to concomitant adaptations due to e.g. exercise, complicatory disease, and life style. The uncertainty involved in the support environment puts specific requirements on the operation of continuous flow assist devices. Suction or over-aspiration should be avoided, and the assist system should meet any conceivable output demands. We believe that a control strategy build on using pulmonary venous return as the feedback variable would suit these requirements appropriately.

The impact of suction on pulmonary circulatory function has received little attention in scientific contributions of the engineering community. In line with the basic functions of the LVAD, i.e. LV unloading and peripheral perfusion, several control strategies have been developed 
with a focus on the output: Stabilization and maximization of LVAD output pressure and flow have been prioritized as the control aims ${ }^{17,35,36}$. Avoidance of suction has been investigated, implemented, and shown to be feasible in a number of papers ${ }^{15-17,26,27,37}$, but the explicit use of measures for pulmonary venous return has not been reported. The current methods for non-invasive measurement of the pressures around the LVAD and the flow through the device could be particularly suitable to implement pump adjustment on the basis of pump interventions as discussed in this paper. The problems with reliable long-term data acquisition rellated to invasiveness, have been tackeled by the use of non-invasive and computational estimation techniques $14,18,29,36,37$.

Preprogrammed periodic manipulations of pump speed may provide a means to monitor the dynamic performance of the assisted circulation over time. As the results of this paper show, specific aspects such as the filling status, the available reserve capacity, and the condition of the rightsided circulation could be evaluated and reported back to either the physician or a knowledge based LVAD controller ${ }^{16,18,37,38}$. Further research and development are mandatory to assess the feasibility, safety, and robustness of such a method.

\section{Limitations}

Although the present study produced interesting data and a detailed perspective on the mechanics of suction, the experimental arm contained definite limitations. The small series $(n=6)$ of experiments were set up as a pilot to evaluate a range of aspects, namely: The feasibility of surgical implantation, basic operation of the device, and the interventions protocol.

The ease of implantation and instrumentation varied with each animal. During the surgical preparations the position of the animal was manipulated and showed to have a considerable effect on hemodynamic status. Furthermore, cardiopulmonary bypass (CPB) was used as a backup, when necessary during implant, and as a means to control circulatory volume. In one animal device implantation could be performed without CPB assistance. In the animal presented with a substantially elevated PVR 
(Fig. 7.2) pulmonary air embolism could not be ruled out. In combination with mild hypovolemia and a minimum LVAD operating speed of 2200 rpm even minor embolism could result in abnormal conditions.

The observed low arterial pressures are associated with the use of CPB. Additionally, left ventricular contractile performance seemed impaired in some animals upon visual inspection. The mechanical load, longitudinal as well as rotational, imposed on the $\mathrm{LV}$ by the stiff inlet cannula and flange sutured to the apex could be responsible for systolic impaiment.

The simulation arm of the study was conceived as a hemodynamic model only. The properties of the model included Starling's law of the heart, pulmonary pressure regulation, baroreceptor response, thoracovascular coupling, and a continuous flow pump characteristic. The dynamics of e.g. neurohumoral feedback, (para-) sympathetic response, renal pressure regulation, and myocardial perfusion were not taken into account. These limitations, as clearly illustrated by Figures 7.3 and 7.7 , are considered responsible for the absence of second order responses. However, the very restrictions that the model design required proved particularly informative in the identification of the relevant factors and mechanisms involved in vivo. The first order congruence of the experimental and simulation results of this study provides strong evidence for the mechanistic nature of suction and its implications.

The clinical significance of the results partly depends on the validity of extrapolating time-course related dynamics like those pictured in Figures 7.3-7.11. Meta-simulations gave the opportunity to study beyond the duration of the acute measurements in vivo (up to 30 minutes). The predictive value of the model must be assumed to decline rapidly with extended simulation durations. However, one may speculate that higher order surrounding mechanisms like those mentioned above will ultimately determine whether or not "latch up", congestion, or suction alter the physiologic function of the patient assisted with a rotary LVAD. 


\section{Acknowledgement}

This study was sponsored by Arrow International Inc., Reading, PA. We want to thank Fabio Leonardi and colleagues at the Center for Veterinary Surgery in Parma for their cooperation and skills.

\section{References}

1 Klotz S, Deng MC, Stypmann J, Roeker J, Wilhelm MJ, Hammel D, scheld HH, Schmid C. Left ventricular pressure and volume unloading during pulsatile versus nonpulsatile left ventricular assist device support. Ann Thorac Surg 2004; 77:143149; discussion 149-150

2 Roell W, Goedje O, Vetter HO, Schmitz C, Dewald O, Reichart B. Improvement of heart-, lung-, and liver-performance during mechanical circulatory support by the Novacor-system. Eur J Cardiothorac Surg 1997; 11:1045-1051

3 Martin J, Siegenthaler MP, Friesewinkel $O$, Fader $T$, wan de Loo $A$, Trummer $G$, Berchtold-Herz $M$, Beyersdorf $F$. Implantable left ventricular assist device for treatment of pulmonary hypertension in candidates for orthotopic heart transplantation-a preliminary study. Eur I Cardiothorac Surg 2004; 25:971-977

4 Salzberg SP, Lachat ML, von Harbou K, Zund G, Turina MI. Normalization of high pulmonary vascular resistance with LVAD support in heart transplantation candidates. European Journal of Cardio-Thoracic Surgery 2005; 27:222-225 Hetzer R, Muller JH, Weng Y - g, Meyer R, Dandel M. Bridging-to-recovery. The Annals of Thonacic Surgery 2001; 71:S109-S113

6 Park SJ, Tector A, Piccioni W, Raines E, Gelijns A, Moskowitz A, Rose E, Holman W, Furukawa $S$, Frazier OH, Dembitsky W. Left ventricular assist devices as destination therapy: a new look at survival. J Thorac Cardiovasc Surg $2005 ; 129: 9-17$

7 Holmari WL, Park SJ, Long JW, Weinberg A, Gupta I, Tiemey AR, Adamson RM, Watson JD, Raines EP, Couper GS, Pagani FD, Burton NA, Miller LW, Naka $Y$. Infection in permanent circulatory support: experience from the REMATCH trial. J Heart Lung Transplant 2004; 23:1359 - 1365

8. Lazar RM, Shapiro PA, Jaski BE, Parides MK, Bourge RC, Watson JT, Damme L, Dembitsky W. Hosenpud JD, Gupta L, Tiemey A, Kraus T, Naka Y.

Neurological events during long-term mechanical circulatory support for heart failure: the Randomized Evaluation of Mechanical Assistance for the Treatment of Congestive Heart Failure (REMATCH) experience. Circulation 2004; $109: 2423-2427$ 

Yeager ML, Buda T, Hoercher KJ, Howard MW, Takagaki M, Doi K, Fukamachi $\mathrm{K}$. Predictors of severe right ventricular falure after mplantable lef tentricular assist device insertion: analysis of 245 patients. Circulation 2002; 106:I198-202 Mebazaa $A$, Karpati $P$, Renaud $\mathbb{E}$, Algotsson L. Acute right ventricular failurefrom pathophysiology to new treatments. Intensive Care Med 2004; 30:185-196

11 Park $\mathrm{CH}$, Nishimura $\mathbb{K}$, Kitano $\mathrm{M}$, Okamoto $\mathrm{Y}$, Ban T. Right ventricular performance is impaired by full assist of left heart bypass. Analysis of right ventricular performance against change in afterload in heart failure models. Asaio J 1994; 40:MBO3-308

12 Reesink K, Dekker A, van der Nagel T, Blom H, Soemers C, Geskes G, Maessen $\mathrm{J}$, van der Veen E. Physiologic-insensitive left ventricular assist predisposes rightsided circulatory failure: a pilot simulation and validation study. Artif Organs $2004 ; 28: 933-939$

13 Ross ]. Cardiovascular system. Chapter 17: Frameworks for analysis of ventricular and circulatory function: Integrated responses. In: West ], ed. Best and Taylor's Physiological basis of medical practice. Baltimore: Williams and Wilkins, 1991; 291-306

14 Trinkl J, Havlik P, Mesana T, Mitsui N, Morita S, Demunck JL, Tourres JL, Monties JR. Control of a rotary pulsatile cardiac assist pump driven by an electric motor without a pressure sensor to avoid collapse of the pump inlet. Asaio J 1993; 39:M237-241

15 Vollkron M, Schima H, Huber L, Benkowski R, Morello G, Wieselthaler G. Development of a suction detection system for axial blood pumps. Artif Organs 2004; $28: 709.716$

16 Holzer S, Scherer R, Schmidt C, Schwendenwein I, Wieselthaler G, Noisser R, Schima $\mathbb{H}$. A clinical monitoring system for centrifugal blood pumps. Artif Organs $1995 ; 19: 708-712$

17 Wu Y, Allaire PE, Tao G, Adams $M_{x}$ Lin $Y$, Wood H, Olsen DB. A bridge from short-term to long-term left ventricular assist device--experimental verification of a physiological controller. Artif Organs 2004; $28: 927.932$

18 Doi K, Golding I.A, Massiello AL, Kopcak MW, Jr., Gerhart RL, Schenk S, lnowe M, Ootaki Y, Fukamachi K. Preclinical readiness testing of the Arrow International CorAide left ventricular assist system. Ann Thorac Surg 2004; $77: 2103-2110$

19 Tuinenga P. SPICE: A guide to circuit simulation and analysis using PSpice. $3 \mathrm{ed}$ London: Prentice Hail International, 1995

20 Cosentino $A$, Martin $\mathbb{R}$, eds. Cardiothoracic interrelationships in clinical practice. Armonk, NY: Futura Publishing Company, 1997 
Mitzner W, Sylvester JT. Hypoxic vasoconstriction and fluid fltration in pig lungs. I Appl Physiol 1981; 51:1065-1071 Ducas J, Sehick U, Girling L, Prewitt RM. Effects of altered left atrial ptessure on pulmonary wascular pressure-flow relationships. Am J Physiol 1988; 255:H19-25 Fang $K$, Krahmer RL, Rypins EB, Law WR. Starling resistor effects on pulmonary artery occlusion pressure in endotoxin shock provide inaccuracies in left ventricular compliance assessments. Crit Care Med 1996; 24:1618-1625 Fishman A. Disorders of the pulmonary circulation. Chapter 82: The pulmonary circulation. In: Fishman A, ed. Fishman's pulmonary diseases and disorders. New York: McGraw-Hill, 1998; 1233-1259

Sun $Y$, Beshara M, Lucariello R], Chiaramida SA. A comprehensive model for right-left heart interaction under the influence of pericardium and baroreflex. Am J Physiol 1997; 272:H1499-1515

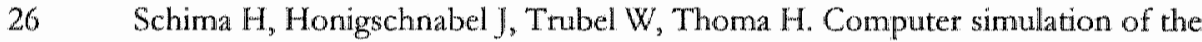
circulatory system during support with a rotary blood pump. ASAIO Trans 1990; 36:M252-254

27 Trinkl J, Mesana T, Havlik P, Mitsui N, Demunck $\ L$, Dion I, Candelon $B$, Monties JR. Control of pulsatile rotary pumps without pressure sensors. ASAIO Trans 1991; 37:M208-210

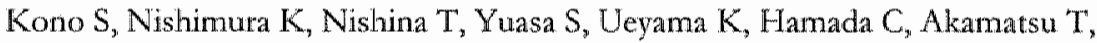
Komeda M. Autosynchronized systolic unlloading during left ventricular assist with a centrifugal pump. J Thorac Cardiovasc Surg 2003; 125:353-360 Schima H, Boehm H, Huber L, Schmallegger H, Vollkron M, Hiesmayr M, Noisser $\mathrm{R}$, Wieselthaler $\mathrm{G}$. Automatic system for noninvasive blood pressure determination in rotary pump recipients. Artif Organs 2004: 28:451 -457 Green J. Fundamental cardiovascular and pulmonaty physiology. 2 ed. Philadelphia: Lea and Febiger, 1987

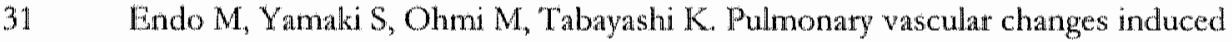
by congenital obstruction of pulmonary venous return. Ann Thorac Surg 2000; 69:193-197 Watzlawick P, Weakland J, Fisch R. Change. Principles of problem formation and problem resolution. New York: Norton, Inc, 1974

33 Gehlbach BK, Geppert E. The pulmonary manifestations of left heart failure. Chest 2004: 125:669-682

34 Wieselthaler GM, Schima H, Dworschak M, Quittan M, Nuhr M, Czerny M, Secbacher G, Huber L, Grimm M, Wolner E. First experiences with outpatient care of patients with implanted axial flow pumps. Artif Organs 2001; 25:331-335 Gitidharan GA, Pantalos GM, Gillars KJ, Koenig SC, Skliar M. Physiologic control of rotary blood pumps: an in vitro study. Asaio f 2004; 50:403 409 
36 Bullister $\mathrm{E}$, Reich S, Shuetz ]. Physiologic control algorithms for rotary blood pumps using pressure sensor input. Artil Organs 2002; 26.931-938

37 Ohuchi $K$, Kikugawa $D$, Takahashi $K$, Uemuna M, Nakamura M, Murakani T, Sakamoto T, Takatani S. Control strategy for totary blood pumps. Artif Organs $2001 ; 25: 366-370$

38 Becker K, Rau G, Kaesmacher $H$, Petermeyer M, Kalfi G, Zimmemann H-j. Fuzzy logic approaches to intelligent alarms. IEEE Engineering in Medicine and Biology 1994:710-716 
156| Chapter 7 
Chapter 8

General discussion 
The previous chapters have presented data and ideas concerning cardiac assist devices and their usage. In this chapter, the contents of the papers will be integrated in the graphical analysis presented in Chapter 1, section 1.4. The discussion will conclude with a summary of the current developments around the studied devices and will provide recommendations for further research in this field.

\subsection{Heart failure: remodeling and reverse remodeling}

The development of heart failure is a complex process in which many factors play a role ${ }^{1,2}$. Eventually, each of these contribute to the disease. Some will counteract the progress of disease, others will promote further decline. Some will be primarily responsible for the undesirable state of affairs, while others merely result from the original fault. Below, a conceptual model is proposed in which the development of disease is presented as a continuous process of adaptation. In this adaptive process (patho-) physiological loading is considered the primordial determinant. The processes of remodeling and reverse remodeling will be used as an example.

\section{Cbronic overload}

In many cases an initial cause for the development of heart failure can be identified, such as myocardial infarction, valve calcification, or hypertension. These etiological causes essentially form a predisposition for heart failure and may be characterized by chronic physiological overload. In Figure 8.1 the previously described intrinsic relationships are depicted. The notion of chronic overload is illustrated by the upward shift of the physiological working range. Furthermore, each relationship is assumed to have a finite gain which poses a limit to what level of performance can be obtained.

\section{Remodeling}

When the heart is predisposed for overload, this will occur more frequently and the heart will adapt to these requirements by remodeling. Initially, cardiac performance will increase to compensate. If overload persists, however, these compensatory mechanisms will tun out of resources and 


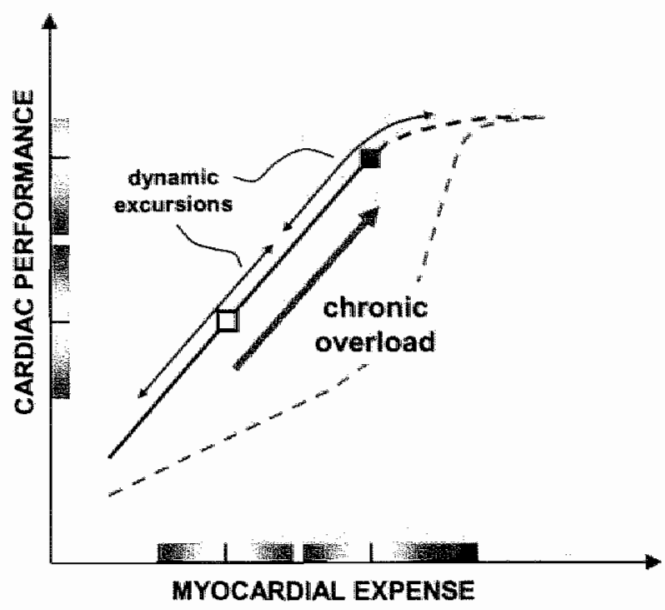

Figure 8.1 Non-linear representation of intrinsic input-output relationships (I). Chronic overload shifts the normal working range of the heart towards its maximum limit. Towards the limit, excessive requirements (expense) are necessary to increase performance (the associated excursions are depicted along the axes).

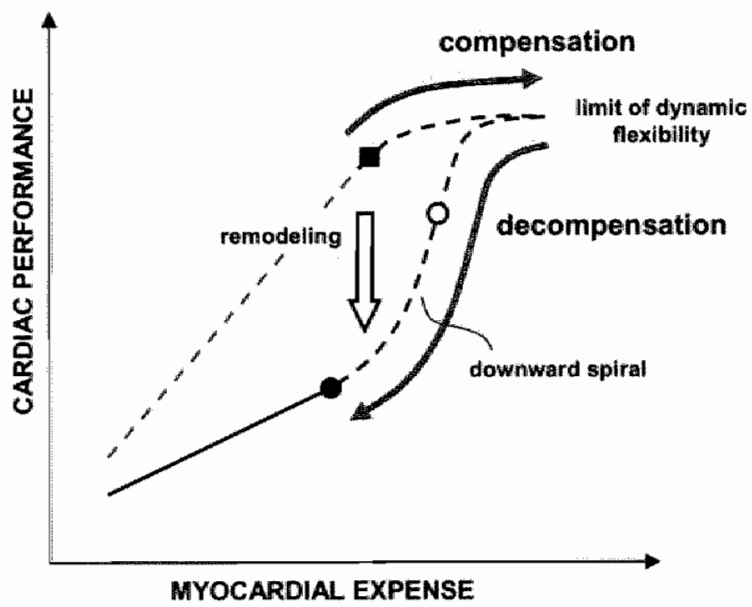

Figure 8.2 Non-linear representation of intrinsic input output relationships (II). Overload is inirially accommodated by compensatory cardiac remodeling of the heart. With persistent overload compensation will fail and progressive decompensation will ensue. The tesultant intrinsic cardiac capacity is decreased. 
decompensatory heart failure may be entered. Not only the heart itself - but the associated homeostatic circuits as well - will be forced to change their attitude to maintain their local balance. During decompensation, however, overall homeostasis can not be achieved because of persistent overload and therefore decompensation is characterized by instability (Fig. 8.2).

Figure 8.2 implies that the original overload condition has switched on the process of degradation, rather than being the cause of failure all the time. Even though the initial incentive for adaptation may not exist any longer (e.g. previous hypertension) an immediate return to the initial state may not occur (e.g. diastolic failure due to cardiac hypertrophy).

\section{State of disease}

When heart failure is established and diagnosed, therapy may be considered. The two-dimensional analysis presented in Figure 8.3 provides two distinct directions in which therapeutic measures may go. Any treatment that increases the loading of the heart may prove beneficial in terms of symptomatic improvement, but will eventually lead to further deterioration due to the prolongation of the overload condition (e.g. inotropic support during cardiogenic shock ${ }^{3,4}$. The alternative option, i.e. to unload the heart, causes increased inactivity and thus provides resolution of the persistent overload condition. In extreme cases, the entire physiological function of the heart can be taken over by mechanical circulatory support (MCS). The resultant complete off-loading is associated with reverse remodeling ${ }^{5-7}$.

Not only MCS is suitable to provide sufficient levels of unloading. Pharmacological intervention and cardiopulmonary rehabilitation have proved to be effective in the treatment of small myocardial infarctions and mild pulmonary hypertension ${ }^{\text {. }}$.

Essentially, in three steps we have arrived at a cyclic pattern that describes the formation of heart disease and provides hints to how the problem may be solved, and even how the disease should not be treated. Now that a framework exists, we will challenge the concept by looking at how it can be applied to the contents of the preceding papers. 


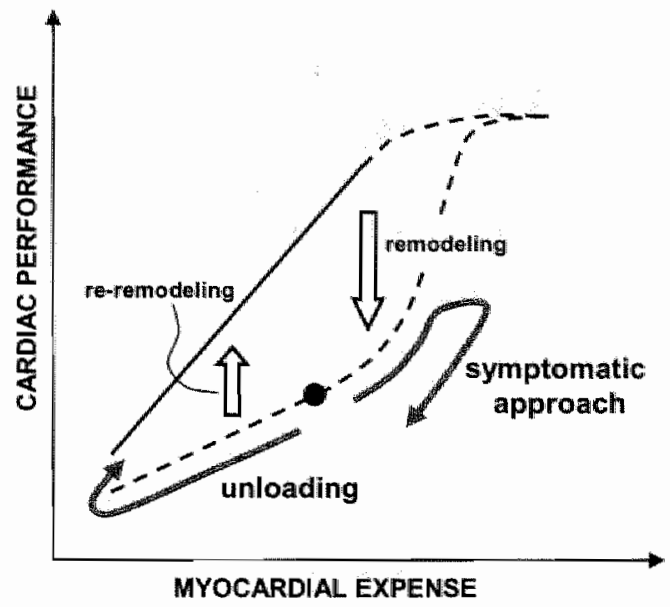

Figure 8.3 Non-linear representation of intrinsic input-output relationships (III). The intrinsic capacity is low which illustrates the heart failure status. The reverse remodeling process is associated with the return to a higher intrinsic capacity. The rationale of (exogenous) support therapies is illustrated by the 'unloading' path: unloading increases the inactivity, which ultimately promotes (endogenous) recovery. The counterproductive effect of therapies that increase cardiac loading is illustrated by the symptomatic approach' path.

\subsection{The young student}

The case report cited in the introduction may illustrate the applicability of the conceptual model.

The final diagnosis was that of an acute myocarditis, which resulted in the arduous clinical course of the patient ${ }^{2}$. His susceptibility to cardiac failure had been increased by the onset of myocardial inflammation (Fig. 8.4). Repetitive overload and gradual deterioration of myocardial function produced the extreme fatigue with which he consulted the GP. From then on, his condition declined rapidly and developed into cardiogenic shock. The inotropic support the patient received did spur the heart towards greater effort in order to maintain vital organ perfusion, but myocardial loading conditions became even worse, now that the amount of work was 


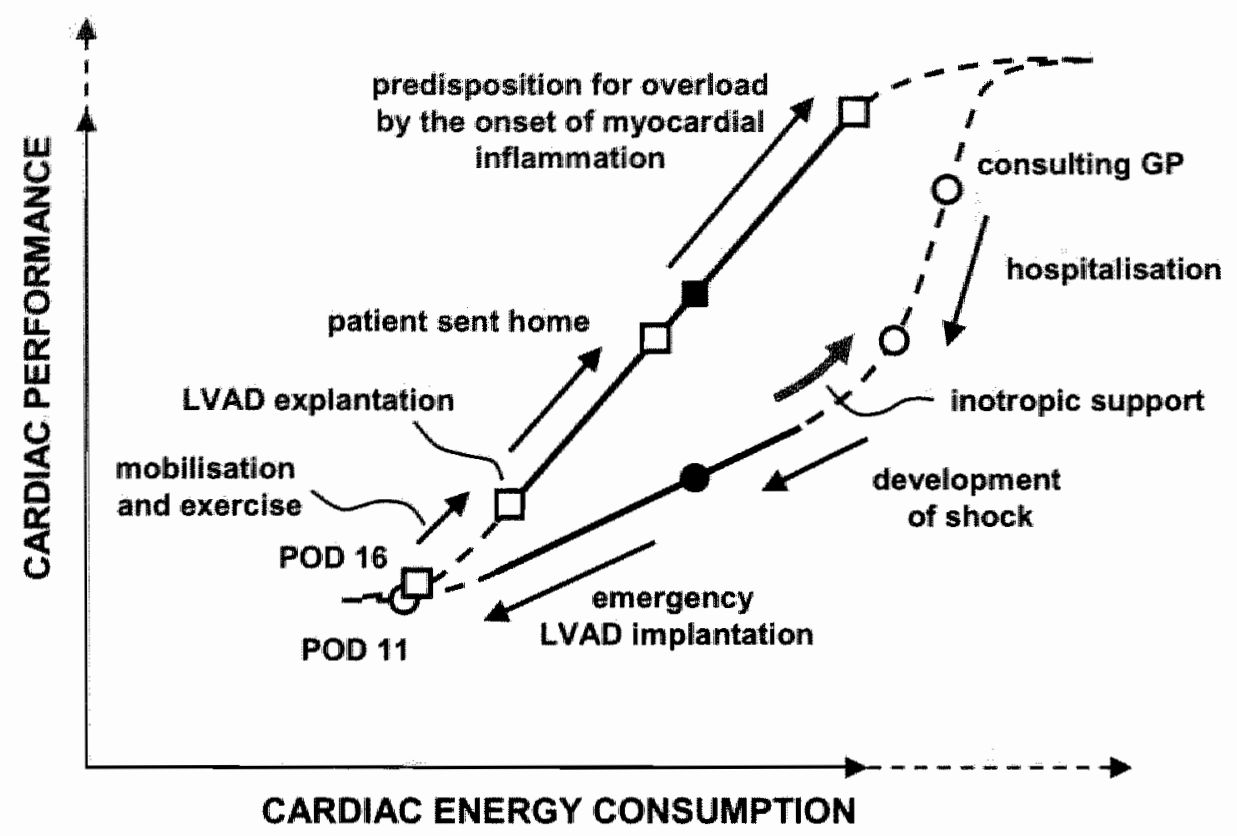

Figure 8.4 The case report: landmarks and events in the dewelopment of acute myocarditis and subsequent LVAD mediated recovery. LVAD, left ventricular assist device; GP, general practitioner; POD, postoperative day.

increased. Progressive deterioration called for an assist device. Ultimately, long term mechanical unloading produced the desired resolution of pump failure. A crucial aspect during support was the repetitious monitoring of cardiac function, which was of aid in deciding when to wean the patient from the device.

\subsection{Hemodynamic collapse due to LVAD suction}

The development of suction due to hypereffective LVAD pumping and its associated problems and resolution is recapitulated briefly in Figure 8.5. It should be noted, that the decision to reduce LVAD flow in anticipation of full suction (see $*$ in Fig. 8.5) in fact prevented the severe deteriotation when full suction would have occurred. The results presented in Chapter 7 also imply a relationship between the area within the loop and the presumed 
changes within the system. The loop area in the computer simulation case was considerably smaller than that of the in vivo experiment (Pig. 7.13). During simulation, only one parameter, the pulmonary venous resistance, changed. In vivo, higher order changes additional to vessel collapse included coronary insufficiency, and systemic vascular responses to hypoperfusion. Interestingly, the recovery process after the pump down intervention, took more time in vivo than shown by the computer simulation. Apparently, loop area, adaptation, and readaptation are closely linked.

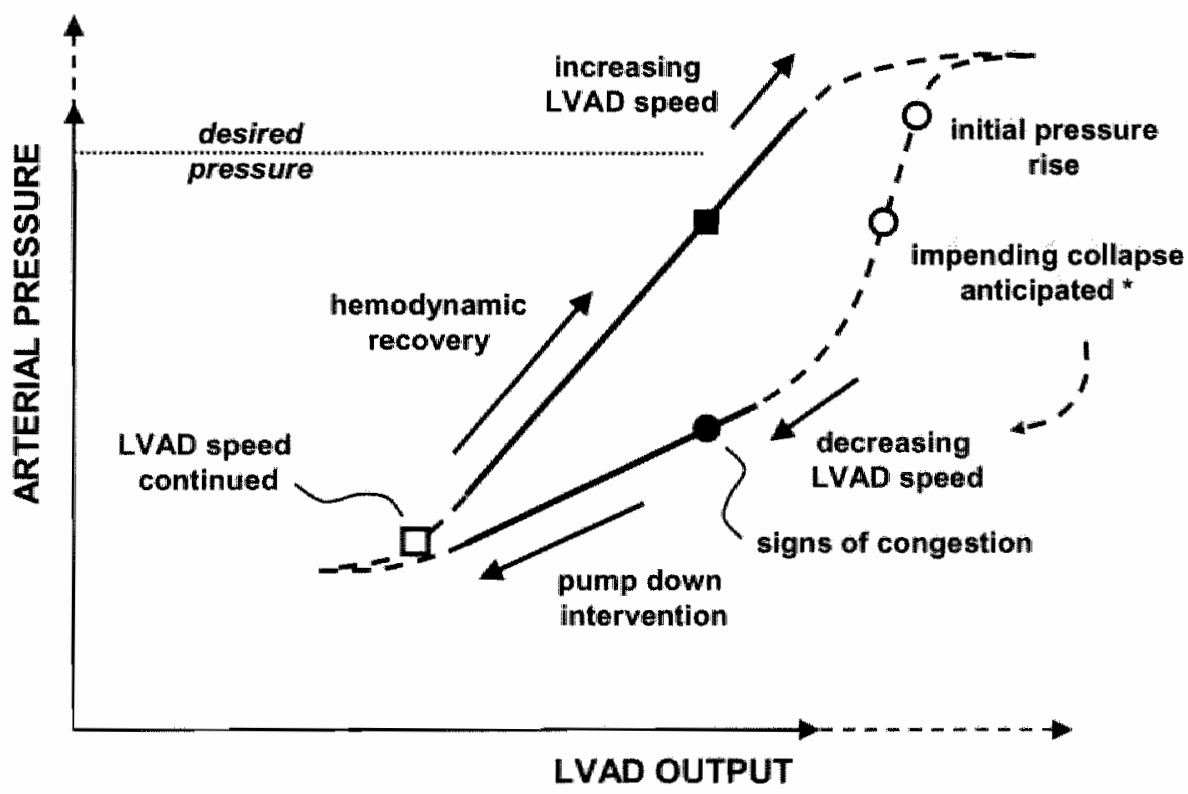

Figure 8.5 The development of hemodynamic collapse due to LVAD suction and the subsequent recovery following a pump down intervention. LVAD, left ventricular assist device. *The decision to decrease LVAD flow was based on the observation of declining inflow pressure (not explicitly incorporated in this analysis); the decision to increase LVAD initially was based on the desire to increase arterial pressure. 


\subsection{Optimization of mechanical support}

In Chapter 3 and 4 the optimization of left ventricular support through countetpulsation was investigated. In these papers on extracorporeal life support, the objective was to obtain a gain in unloading without compromising peripheral perfusion. In Chapter 6 , the unloading capability of an intracardiac device was shown to be greater than that of the intraaortic device. Unloading may be the only method of saving viable myocatdium in case coronary perfusion is impeded due to e.g. coronary lesions. The increase in unloading in the therapeutic context is illustrated in Figure 8.6.

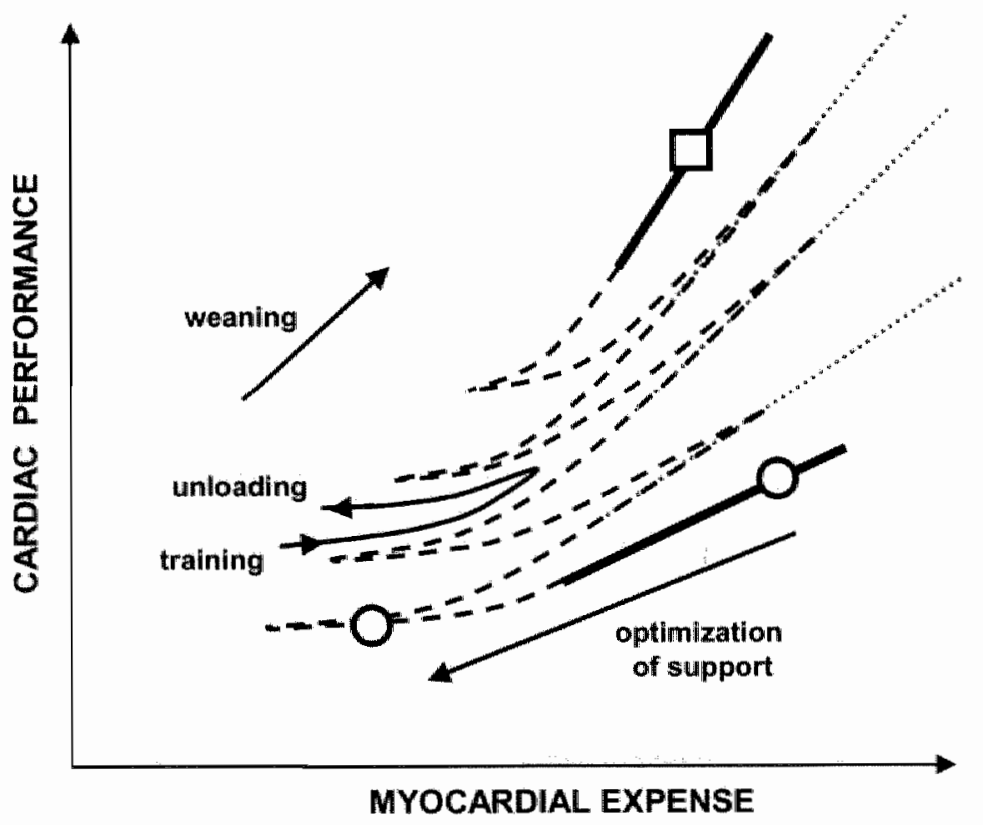

Figure 8.6 Optimization of support for cardiac unloading. The transition from the disease relationship towatds the healthy curve runs via intermediate, transitory states (grey dotted lines). The repetitious mechanical unloading and training pattern is related to these stages of partial cardiac recovery. 
The rationale behind the use of these devices is that cardiac recovery can be promoted or facilitated by decreasing the workload of the heart. Clearly, the unloading capacity required from the device depends on the degree of compromise present in the cardiac system. It remains however difficult to exactly determine the necessary amount of unloading ${ }^{10}$. Furthermore, full unloading alone does not lead to full recovery as it is associated with atrophy and limited reserve capacity ${ }^{8,11}$. Both the "titration" of unloading and the demand for training can be accommodated by switching the operation mode of a cardiac assist device. An impression of this dynamic interaction between device and heart is given in Figure 8.6. Here, unloading and training for recovery are associated with the weaning process ${ }^{6.11}$.

\subsection{Characteristics of the conceptual model}

In the previous paragraphs, we have applied a graphical approach to the analysis of heart failure and its treatment through mechanical circulatory support. By way of this method different aspects such as remodeling, physiologic overload, unloading, training, and the weaning problem have been shown to be interrelated. In Figure 8.7, a generic conceptual model is shown in which the cyclic pattern of change within 'a system' is described. Here, the six aspects that determine its applicability are briefly discussed.

\section{Noise}

If we are to track any change in the intrinsic property of a system, we should be able to quantify the relationship between the system's variables accurately. This implies that measurements need to be of sufficient numerical resolution and should be performed at an appropriate frequency ${ }^{12}$. However, the ultimate degree of accuracy that can be obtained will be limited by noise. Noise is generated by the natural stochastic variability of dynamic processes and the physical limitations involved in the sensory process, e.g. what one can not hear, see, etc.; transducer noise; limited data storage capacity; heat production. The impact of noise may be reduced by filtering data and by increasing the impact of measurement stimuli. For instance, when an ill or recovering heart is not challenged to perform a 
certain amount of work, no pertinent evaluation of its contractile function can be made ${ }^{10,13}$.

\section{Predisposition}

Under normal working conditions, the occurence of overload will be less frequent than when the system is compromised by chronic overuse or by a defect. In the current framework these predispositions are thought to cause no adaptation by themselves. Examples of predisposing factors for ventricular failure are for instance: the calcification of an aortic valve, hypertension. Often 'predisposition' may be synonymous to the 'etiology' of a disease.

\section{Adaptation}

When persistent (over-) loading exists, a system will respond by changing its intrinsic properties to cope with the increase in demand ${ }^{2,14}$. These changes may establish an increase in strength - or rather sensitivity - of the system to comply with demands, as is the case with e.g. ventricular hypertrophy. If this type of change is not possible, the system might change its attitude towards a lesser performance in order to maintain its own homeostasis (e.g. myocardial stunning and hibernation ${ }^{3,15}$ ). The greater system, however, is still required to perform according to demand and thus decompensation may ensue in spite of the adaptive effort.

Section 8.4 already addressed the subject of reversibility and the associated transitory states. The path along the intermediate curves (Fig. 8.7) expresses the dynamic nature of the adaptation and recurrent overload processes. It is important to take in account the time factor involved. If overload is only of short duration, little or no measurable adaptations may occur. With longstanding stimuli the number of small, consecutive changes will increase as well as their persistence upon resolution of the stimulus. The incorporation of transitory curves and the resultant area of the loop provide a graphical expression of the concept of adaptation (see also section 8.3).

A final remark should be made concerning the adaptive process. Change may occur either within the system of interest or its environment. 
Since the performance of the system is the inescapable result of its interaction with its environment, the study of adaptation and its reversal through the use of therapy (which is an environmental factor) should include the dynamic behavior of both system and environment ${ }^{1,16}$.

\section{Point of paradox}

When regarded in isolation, the state of disease is characterized by paradoxical behavior. The usual remedy for a lack of performance, i.e. to increase expense, does not only fail to obtain the desired performance but may even be counterproductive. In the example of the young man's myocarditis the use of inotropic support did help to satisfy systemic perfusion requirements for a while, but ultimately failed to promote the

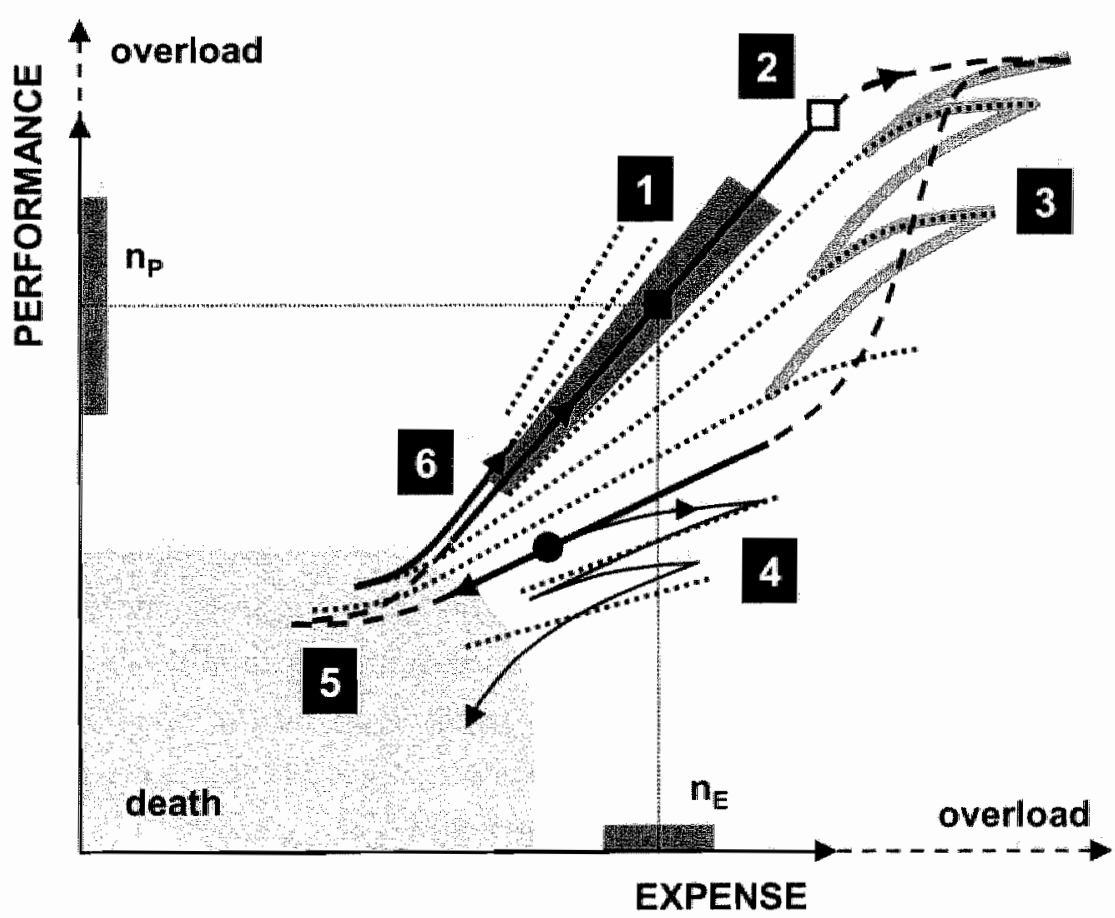

Figure 8.7 The characreristic aspects of the cyclyc partern in the conceptual model. 1: noise, 2: predisposition, 3: adaptation, 4: point of paradox, 5: readaption, 6: path uniqueness. $\mathrm{n}_{\mathrm{E}}$ and $\mathrm{n}_{\mathrm{p}}$, noise-band projections on the input (expense) and output (performance) axes. 
recovery of the myocardium. Another example that illustrates this concept is the ambiguous result obtained with revascularization. After such procedures, the heart hardly ever retains its function immediately and the direct exposure to a full workload may induce failure again 3,15 .

Apparently, 'more of the same' does not work in some cases, which calls for a change in scope. The paradox is untied by looking at the dynamic interaction of the system (whether it is a myocyte, a heart, or a LVAD) with that of its environment in the past and the present. The view as presented in Figure 8.7 connects the disease state with the preceding deterioration due to the incidence of overload, and thereby provides an explanation for the failure of the more-of-the-same approach. Consequently, this provides a clear rationale for those therapies seeking recovery through unloading. From section 8.3 we learned that hypoperfusion due to LVAD suction may only find a solution in decreasing pump flow, as opposed to the intuitive boost. Around the point of paradox 'less is more' seems appropriate ${ }^{16}$.

\section{Readaptation}

The applicability of rehabilitation techniques is unquestionably linked to the premise that reverse adaptive processes exist and that they can be brought about by external intervention ${ }^{17}$. When reversibility is assumed, then the same aspects discussed under adaptation apply to this 'corner' of the loop (Fig. 8.7), under the connotation that 'overloading' becomes 'unloading' and that 'deterioration' becomes 'augmentation', and so forth. In practice, psychological, economical, and organizational issues may limit the rigorous pursuit of readaptation. The mobilization and exercise in cardiopulmonary rehabilitation are good examples of therapies that seek durable recovery ${ }^{8.18}$. The time required for full recovery may be less than the development of the disease involved, or may exceed this. Presumably, an appropriate rule of thumb may be 'recovery time $\approx$ time to disease' (Chapter 7 ).

\section{Path uniqueness}

The cyclic pattern is actually not perfectly closed. In general, when recovery has been obtained, the gain relationship will not be exactly the same as 
before. The complexity of the human biological system and the multitude of interactions with its environment provide an infinite amount of possible states that can be marked as 'healthy'. The nature of this can be best described by the notion that 'through change an adaptive organism learns" $1,2,16,19$.

\subsection{Comments}

Our initial graphical analysis only used two variables, namely expense and performance. Evidently, these are only concepts expressing the net outcome of clusters of measurable variables. In practice, multiple variables define a system's state of operation. The cyclic pattern is recursive: any shift from one intrinsic relationship to another within the greater cycle may be regarded as cyclic as well. This property sustains the rationale for joint unloading and training in the weaning process (Figure 8.6). The proposed concept is largely qualitative in nature. For practical applications the quantitative validity is to be demonstrated. The work presented in Chapter 7 illustrates the numerical application of the conceptual model. The pursuit of our graphical analysis has provided a useful conceptual model for studying reversible heart failure and the role of mechanical circulatory support. It might be interesting to investigate whether the conceptual model can be applied to the evaluation of organizational, psychological, and economical processes of change ${ }^{1,16}$.

\subsection{Future work}

The current state of technology has provided numerous examples of reliable, biocompatible, and versatile blood pumps. The application of these devices is receiving more and more critical attention from engineering, experimental, and clinical communities. Still, further research is necessary to increase the safety and efficacy of artificial hearts.

With rotary ventricular assist devices the sensitivity to demand may be increased by feedback control. The robustness of the control should be of primary concern. Furthermore, the effect of pump suction on organ 
function, blood integtity, and safety has not been investigated to great extent. The results on LVAD suction presented in this thesis provide a clear incentive for the study of mallicious pump interactions in the field of ECLS and ordinary cardiopulmonary bypass.

With any support system the ultimate goal is to wean the patient from the device (destination therapy excluded). A vast amount of knowledge is still to be acquired or is only implicitly present in the minds of experts. Study of the dynamic interactions between the pathological circulation and the mechanical support system may be helpful to improve weaning success tates and long term outcome. This thesis has contributed its share on the functional and hemodynamic level. Future investigations should include the molecular, cellular, and organic levels as well. Presently, a method for advanced monitoring and training purposes through intentional. pump interventions is under development.

\subsection{Conclusion}

The papers in this thesis have touched upon the technological development, physiological interaction, optimization of support, diagnostics, and clinical use of mechanical circulatory support. The pressure sensing system evaluated in Chapter 2 is presently commercially exploited as an invasive diagnostic tool and a triggering and monitoring device for the intra-aortic balloon pump. Accurate pressure measurements have contributed greatly to the experimental work presented in this thesis. The improvement of support through timing optimization, as described in Chapter 3 and 4, relies strongly on the availability of stable and accurate pressure sensing techniques. The use of mechanical unloading as a treatment for heart failure is gaining interest worldwide as experience grows with bridging to recovery. Chapters 3-7 show how mechanical unloading can be achieved and improved, but they also addressed the potentially counterproductive effects. The analyses of the interaction between assist device and the native circulation suggest that under pathological conditions the assisted circulation as such may behave in a non-linear fashion. This non-linear 
behavior has a cyclic pattern, which appears to apply to the reversible disease process as well.

\section{References}

1 Bateson G. Steps to an ecology of mind. 2 ed. New York: Ballantine Books, 2000

2 Ashby W. Design for a brain. New York: Wiley, 1952

3 Hollenberg SM, Kavinsky CJ, Parrillo JE. Cardiogenic shock. Ann Intern Med $1999 ; 131: 47-59$

4 Hollenberg SM. "For every complex problem, there is a solution that is simple...and wrong" . Crit Care Med 2000; 28:3088-3089

5 Thohan V, Stetson SJ, Nagueh SF, Rivas-Gotz C, Koemer MM, Lafuente JAn Loebe M, Noon GP, Torre-Amione G. Cellutar and Hemodynamics Responses of Failing Myocardium to Continuous Flow Mechanical Circulatory Support Using the DeBakey-Noon Left Ventricular Assist Device: a Comparative Analysis With Pulsatile-Type Devices. J Heart Lung Ttansplant 2005; 24:566-575

6 Muller J, Wallukat $G$, Weng YG, Dandel M, Spiegelsbetger S, Semrau S, Brandes $\mathrm{K}$, Theodoridis $\mathrm{V}$, Loebe $\mathrm{M}$, Meyer $\mathrm{R}$, Hetzer $\mathbb{R}$. Weaning from mechanical catdiac support in patients with idiopathic dilated catdiomyopathy. Circulation 1997; 96:542-549

7 Hon $J \mathrm{~K}$, Yacoub MH. Bridge to recovery with the use of left ventricular assist device and clenbuterol. Ann Thorac Surg 2003; 75:S36-41

8. Pozeh B, Duncan K, Krueger S, VerMaas P. Adjunctive effects of exercise training in heart failure patients receiving maximum pharmacologic therapy. Prog Cardiovasc Nurs 2003; 18:177-183

9 Holman WL, Bourge RC, Kirkin JK Case report: circulatory support for seventy days with resolution of acute heart failute. J Thorac Cardiovasc Surg 1991; 102:932-934

10 Balady GJ, Larson MG, Vasan RS, Leip EP, O'Donnell CI, Levy D. Usefulness of exercise testing in the prediction of cotonary disease risk among asymptomatic persons as a function of the Framingham risk score. Circulation 2004; 110:19201925

11. Yacoub MH, Tansley P, Birks EJ, Banner NR, Khaghani A, Bowles C. A novel combinarion therapy to reverse end-stage heart failure. Transplant Proc 2001; 33:2762-2764

12 Goyal D, Macfadyen RJ, Watson RD, Lip GY. Ambulatory blood pressure monitoring in heart failure: a systematic review. Eur J Heat Fail 2005; 7:149-156 
13 Colson $P$, Ryckwaent $F$, Saussine $M$, Ferriete $M$, Mbat B. Montoring weaning from BVAD Thotatec with peak orygen consumption. Ann Thorac Surg 2004; 77:1808-1810

14. Bateson $G$. The role of somatic change in evolution. Steps to an ecology of mind. New York: Ballantine Books, 2000; 533

15 Mangano DT. Myocardial stunning: an overview. J Card Surg 1993; 8:204-213

16 Watzlawick P, Wealkland J, Fisch R. Change. Principles of problem formation and problem resolution. New York: Norton, Inc; 1974

17 Shavit $A$, Gutfinger $C$. Heat engines and the second law of themodynamics. Thermodynamics. From concepts to applications. London: Prentice Hall, 1995; $108-125$

18 Gordon NF, Gulanick M, Costa F, Fletcher G, Franklin BA, Roth Ej, Shephard T. Physical Activity and Exercise Recommendations for Stroke Survivors: An American Heart Association Scientific Statement From the Council on Clinical Cardiology, Subcommittee on Exercise, Cardiac Rehabilitation, and Prevention; the Council on Cardiovascular Nursing; the Council on Nutrition, Physical Activity, and Metabolism; and the Stroke Council. Circulation 2004; 109:2031. 2041

19 Jain S. Systems that learn: an introduction to leatning theory. Cambridge, MA: MIT Press, 1999 


\section{Summary}

Mechanical cardiac support plays an important role in the treatment of patients with end-stage heart failure. Currently, the most promising options are the use of mini heart-lung machines and rotary ventricular assist devices. The performance of these pump systems is reliable and effective technically, but the physiological interface with the circulation requires optimization. The interaction between a mechanical support system and the native circulation is the subject of this thesis.

In Chapter 2, the experimental validation of an optical blood pressure measurement system is described. Upon comparison with existing electrical transducers, the optical prototype showed comparable dynamic response characteristics and stability but a superior electromagnetic disturbance rejection. Currently, the system is used for the monitoring and triggering of intra-aortic balloon pumps.

In today's mini cardiopulmonary bypass circuits continuous flow technology is employed because it offers reliability and cost-effectiveness. However, if cardiac recovery is the target of treatment, these systems may not be suitable to control myocardial working conditions sufficiently. In Chapter 3, the pulse interaction between a pulsatile cardiopulmonary bypass circuit and the native left ventricle is studied in calfs. The results show that systolic co-pulsation is associated with increased left ventricular oxygen consumption while counterpulsation improves the myocardial oxygen balance. Further research is necessary to develop clinical protocols that enable the adjustment of mechanical cardiac unloading to the specific patient's needs. The use of intra-aortic balloon counterpulsation during non-pulsatile cardiopulmonary bypass is described in Chapter 4. In this study, the efficacy of balloon counterpulsation during femoral cannulation was compared to the thoracic cannulation case. In both cases, intra-aottic balloon pumping improved myocardial working conditions. In the femoral case, however, the diastolic augmentation of aortic pressure was attenuated while left ventricular afterload reduction was diminished with thotacic 
cannulation. Several clinical studies have shown the benefit of counterpulsation in partial cardiopulmonary bypass patients.

The development and validation of a computer model of the assisted circulation is described in Chapter 5. In this model, worst-case hemodynamic interactions between a rotary left ventricular assist device (LVAD) and the pulmonary circulation were simulated. The results support the existing theory and clinical evidence for right heart overload, in case of preload insensitive left ventricular unloading. The measurements and interventions were repeated in a calf. Both models showed good correlation under baseline and pulmonary hypertensive conditions, but responded differently to an increased filling intervention. Further development of the hemodynamic computer model should include the inclusion of a left ventricle and a systemic vascular bed.

In Chapter 6, the hemodynamic efficacy of a new intracardiac LVAD is compared to intra-aortic balloon pumping (IABP) in a bovine model of acute mitral insufficiency. The intracardiac device showed significantly better unloading properties than the balloon pump while IABP enhanced myocardial perfusion more than the impeller pump. The new device could fill the gap in the current range of support devices. A combination of the two concepts in one device seems promising.

The effect of totary LVAD suction on the circulatory function is investigated in Chapter 7. Acute suction events were simulated in a hemodynamic computer model and in sheep. The results show that a transient suction event may give rise to prolonged hemodynamic insufficiency and reduced device efficacy, depending on the presence of pulmonary circulatory pathophysiology. The use of dynamic pump interventions appears to be a promising tool, to both detect suction and to overcome the associated problems.

In Chapter 8, a conceptual model is developed in which the development of heart failure and the role of mechanical cardiac support as a treatment are brought into relationship. 


\section{Samenvatting}

$\mathrm{Bij}$ de behandeling van patienten met terminaal hartfalen speelt mechanische ondersteuning van het hart een belangrijke roll. Het meest veelbelovend zijn de mini hart-longmachine en het rotatie-steunhart van het roterende pomp type. Technisch gezien presteren de huidige pompen effectief en betrouwbaar, maar de functionele koppeling met de bloedsomloop is nog niet optimaal. Het onderwerp van dit proefschrift is de interactie tussen het mechanische ondersteuningssysteem en de natieve bloedsomloop.

In hoofdstuk 2 wordt de experimentele test van een optisch bloeddrukmeetsysteem beschreven. Het prototype van de optische sensor bleek een dynamische responskarakteristiek en een stabiliteit te hebben die vergelijkbaar was met bestaande sensoren. De electromagnetische storingsongevoeligheid van de optische sensor was echter veel groter. Het systeem wordt nu gebruikt voor het monitoren en aansturen van intraaortale ballon pompen.

In de huidige mini hart-longmachines worden konstantestromingspompen gebruikt, omdat deze betrouwbaar zijn en kosteneffectief. Deze systemen zouden minder geschikt kunnen zijn voor het reguleren van de werkomstandigheden van de hartspier, indien het herstel van het hart wordt beoogd. In hoofdstuk 3 wordt een dierstudie naar de interactie tussen de drukgolven van een pulserende hart-longmachine en de linker hartkamer beschreven. De resultaten laten zien dat de gelijktijlige ejectie van de pomp en het hart de zuurstofconsumptie van de linker hartkamer verhoogt, terwijl uit-fase ejectie juist de balans tussen zuurstof aanbod en verbruik verbetert. Voortgezet onderzoek is nodig om klinische protocollen te ontwikkelen die het mogelijk maken, om de mechanische ontlasting van het hart aan te passen aan de individuele patient. Het gebruik van intra-aortale ballon tegenpulsatie tijdens het ondersteunen met een nietpulserende hart-longmachine is bestudeerd in hoofdstuk 4 . In deze studie is gekeken naar de effectiviteit van ballon tegenpulsatie bij verschillende cannulatie methoden, namelijk die via de grote vaten en via de liesvaten. In 
beide gevallen verbeterde intra-aortale ballon tegenpulsatie de werkomstandigheden van de hartspier. Bij cannulatie via de liesvaten was de verhoging van de aortadruk tijdens de rustfase van het hart verminderd effectief. Hetzelfde kenmerkte de drukontlasting van de hartkamer maar dan in het geval van cannulatie via de grote vaten. Een aantal klinische studies heeft de voordelèn van tegenpulsatie gedurende de gedeeltelijke overname van de hart-longfunctie reeds aangetoond.

In hoofdstuk 5 wordt de ontwikkeling en validatie beschreven van een computer model dat de ondersteunde bloedsomloop representeert. Met dit model werd de interactie tussen een rotatie-steunhart en de longcirculatie gesimuleerd onder 'worst-case' condities. The resultaten ondersteunen de bestaande theorie en klinisch bewijs voor het optreden van overbelasting van de rechter harthelft ten gevolge van een mechanische hartkamer ontlasting die ongevoelig is voor aanbod. De metingen en interventies werden herhaald in een proefdiermodel. Beide modellen lieten een goede correlatie zien onder basale en pulmonaal hypertensieve condities, maar reageerden verschillend op het vergroten van het vullingsvolume. De verdere ontwikkeling van het hemodynamische computer model zou gericht moeten zijn op het inbouwen van een linker hartkamer en het systemisch vasculaire bed.

In hoofdstuk 6 wordt de hemodynamische effectiviteit van een nieuw intracardiaal hulppompje vergeleken met die van de intra-aortale ballon pomp (LABP) in een proefdiermodel waarin mitraalkleplek werd aangebracht. Het intracardiale pompje bleek beter in staat het hart te onlasten dan de IABP, terwijl de doorbloeding van de hartspier meer toenam tijdens het gebruik van de IABP. Het nieuwe pompje zou de leemte in het huidige arsenaal van ondersteuningssystemen kunnen vullen. Een combinatie van beide concepten in één product lijkt veelbelovend.

Het effect van zuiging bij rotatie-steunharten op de functie van de bloedsomloop wordt onderzocht in hoofdstuk 7. Acute episodes van overmatige zuiging werden gesimuleerd in een hemodynamisch computer model en in schapen. De resultaten laten zien dat, afhankelijk van de aanwezigheid van een afwijkende longcirculatie, een voorbijgaande periode van overmatige zuiging een voortdurend ontoereikende hemodynamische 
status en een verminderde pompeffectiviteit kan veroorzaken. Dynamische pomp interventies lijken een interessante mogelijkheid te bieden om zuiging te detecteren en om de problemen als gevolg van overmatige zuiging op te lossen.

In hoofdstuk 8 wordt een conceptueel model gepresenteerd waarin het ontstaan van hartfalen en de rol van mechanische ondersteuning bij de behandeling daarvan met elkaar in verband worden gebracht. 


\section{Dankwoord}

'Boekje klaar!' Maar niet zonder meer... De promotieperiode is een aaneenschakeling van projecten, reizen, mensen, lezen en schrijven, maar ook van vallen en opstaan. Alleen red je het niet, of is het gewoon niet bevredigend. De een was boegbeeld, de ander strohalm, weer een ander 'een aai over de bol', maar een ieder die me tijdens de afgelopen tijd heeft gesteund, geholpen of simpelweg deelgenoot is geweest wil ik hierbij hartelijk bedanken.

Een aantal van hen zou ik echter kort met naam willen noemen:

Erik, als co-promotor, collega en luisterend oor heb je altijd interesse getoond voor wat mij bewoog. Je hebt me bovenal de ruimte en de kans gegeven mijn eigen weg te vinden, en dat kan ik ten zeerste waarderen! Bedankt voor het mogelijk maken van de onderzoeksprojecten, de reizen, en je steun en suggesties bij het schrijven van de manuscripten. Jos, je hebt als promotor, ondanks je drukke agenda, de grote lijnen van het onderzoek gestalte gegeven. Bedankt voor je kritiek, suggesties en vertrouwen. Theo, improviseren is 'your middle name'; zonder dat waren een hoop metingen voor niks geweest; onze reizen zijn altijd goed gebleken voor een part sterke, maar echt gebeurde verhalen en zo'n vijf kilo kaas en salami. Bedankt voor de 'zwarte dozen', ik heb er dierbare herinneringen aan! André, kritische co-auteur en collega; bedankt voor het schtappen en meedenken, en voor het wijzen van 'Mango's', het jaar daarna heb ik dat goed kunnen gebruiken! Loes, bedankt voor je inzet en je bijdragen aan de twee papers; 32 kanalen data acquisitie in één keer foutloos aansluiten, dat is geloof ik nog niemand gelukt, maar jou wel! De overige co-auteurs, bedankt voor de plezierige samenwerking, jullie suggesties en kritick. Dennis, vriendschap, nuchterheid en prettig klankbord; bedankt voor de nodige koffie-dates en kook-avonturen, en dat ik je aan mijn zijjle mag hebben tijdens de verdediging! Thomas, mijn kamergenoot (op elk van de viif verschillende werkplekken binnen twee jaar die ik heb mogen aandoen); 
samen schrijven was zowel inspirerend alsook hilarisch op momenten; bedankt dat je mijn paranimf wilt zijn. De studenten, Alina, Annerie, Antoine, Caroline, Jack, Jasper, Robert, William, Wim: me dunkt, toch een hele lijst! Bedankt voor jullie bijdragen en inzet. Monique, bedankt voor je steun en enthousiasme! Jo, kamergenoot van het eerste uur, bedankt. De staf van de afdelingen Cardiothoracale Chirurgie, Extracorporale Circulatie zou ik willen bedanken voor hun hulp en de prettige samenwerking. Bas, bedankt voor je interesse en vertrouwen. De dames van het secretariaat, bedankt voor jullie hulp. Lysette, extra bedankt voor je inzet en steun tijdens de laatste loodjes en rond de aanstellingsperikelen! Barry, bedankt voor de software; zonder dat geen boekje...

Bij deze wil ik de beoordelingscommissie, Theo Arts, Ton Gorgels, Bart Meyns, Mark Post en Frans van de Vosse, bedanken voor hun interesse en goedkeuring.

Bedankt voor jullie steun, creativiteit en vriendschap: Michiel, Patrick, Ruud, Hein, Matthias, Arthur, Del Foob, Pinkie, Arjen, Carlo, Michael, Geert, Ron, Agnes, Linda, Natasja, de familie Curfs, en wiens naam ik nu even niet kan opkomen.

$\mathrm{Pa}, \mathrm{ma}$, This en Ard, bedankt voor jullie steun, liefde, and the occasional schop onder het achterwerk. Een dikke knuffe!!

Daan, lieverd, ik hoop dat je nog lang mijn 'co-auteur' blijft. Two bearts are better than one... 


\section{Curriculum Vitae}

Koen Damiel Reesink

born May 19, 1976 in Eindhoven, The Netherlands

Education

1988-1994 Gymnasium $\beta$, Eindhorens Protestants Lyceum, Eindhoven

1994-1998 BSc Electrical Engineering, Fontys Hogeschool, Eindhoven

1998-2002 MSc Electrical Engineering, Eindhoven University of

Technology. Acquisition of 1998 TUe/industry scholarship

Research

1997 Student-researcher, Innovation Centre, Wire Wound

Components division, Philips N.V.

1998-2002 Student-researcher, depts. of Cardiology and Cardiothoracic

Surgery, University of Maastricht

2002-2005 Part-time PhD-student, dept. of Cardiothoracic Surgery,

University of Maastricht (dr.ir. F.H. van der Veen, Prof.dr.

J.G. Maessen)

2005- Postdoctoral fellow, dept. of Biophysics, University of

Maastricht (Prof.dr.ir. A.P.G. Hoeks)

\section{Extracurricular}

1996-

Semi-professional drummer and band member in the Sonic

Blast Big Band, Eighty Eight, Del Foob, and various Jazz

and Rock combos. International performances in the United

Kingdom, Poland, France, Germany, Zwitserland, and

Singapore. 


\title{
Publications
}

\author{
Articles
}

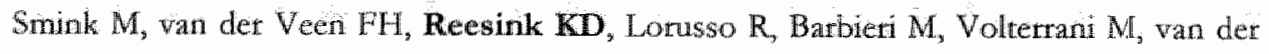
Nagel T, Carraro U. Imaging of skeletal muscle contraction after cardiomyoplasty. Basic Appl Myol 1999; 9:223-227

Reesink KD, van der Nagel T, Bowelander J, Jansen JR, wan der Veen FH, Schreuder JJ. Feasibility study of a fiber-optic system for invasive blood pressure measurements. Catheter Cardiovasc Interv 2002; 57:272-276

Dekker $A$, Reesink K, van der Veen $\mathbb{E}$, Van Ommen V, Geskes $G$, Soemers C, Maessen J. Efficacy of a new intraaortic propeller pump rs the intraaortic balloon pump: an animal study. Chest 2003; 123:2089-2095

Dekker AL, Reesink KD, vath der Veen FH, van Ommen GV, Geskes GG, Soemers $A C$, Maessen $J G$. Intra-aottic balloon pumping in acute mitral regurgitation reduces aortic impedance and regurgitanr fraction. Shock $2003 ; 19: 334-338$

Reesink K, Dekker A, van der Nagel T, Blom H, Soemers C, Geskes G, Maessen J, van det Veen E. Physiologic-insensitive left ventricular assist predisposes tight-sided circulatory failure: a pilot simulation and validation study. Artif Organs 2004; 28:933-939

Reesink KD, Dekker AL, Van Ommen V, Soemers C, Geskes GG, van der Veen FH, Maessen JG. Miniature intracandiac assist device provides more effective cardiac unlonding and circulatory support during severe left heart failure than intraartic balloon pumping. Chest $2004 ; 126: 896-902$

Reesink KD, Sauren LDC, Dekker AL, Severdija E, wan der Nagel T, Geskes GG, wan der Veen FH, Maessen JG. Synchronously counterpulsating extracorporeal life support conances myocardial wotking conditions regardless of systemic perfision pressure. Eur J Cardiothorac Surg 2005; in press 


\section{Published abstracts}

Dekker AL, Reesink KD, wan der Veen $\mathrm{FH}_{3}$ van Ommen GV, Geskes $G \mathrm{G}$, Soemers $\mathrm{AC}_{\text {, }}$ Maessen JG. The new jomed reitan catheter pump ws. iabp during acute mitral regurgitation. Neth Heart ] 2002; 10 (Suppl 1):24

Dekker AL, Reesink KD, van der Veen FH, van Ommen GV, Geskes GG, Soemers AC, Maessen JG. Comparison of IABP and the new Jomed Reitan catheter pump in acute mitral regurgitation. Eur Heart ) 2002; 23 (Abstract suppl):710

Geskes GG, Maessen JG, Dekker AL, Reesink KD, Soemers AC, van der Veen FH, wan Ommen $G V$. The new jomed reitan catheter pump vs. iabp during acute mitral regurgitation. Heart Surg Forum 2002; 5:S176

Reesink KD, Dekker $A L$, van der Nagel $T$, van der Veen FH, van Ommen GV, Ganushchak Y, Geskes GG, Soemers AC, Maessen JG. New Impella intracardiac minipump supports the left heart significantly better than aortic balloon pumping. Neth Heart J 2002; 10:485

Reesink KD, Dekker $A L$, van der Nagel $T$, van der Veen $F H$, wan Ommen $G V$, Ganushchak Y, Geskes GG, Soemers AC, Maessen JG. New Impella intracardiac minipump supports the acutely failing left heart significantly mote effective than intraortic balloon pumping. J Am Coll Cardiol 2003; 41 (6 Suppl A):215A

Reesink KD, Dekker AL, wan der Nagel $T$, van der Veen FH, van Ommen $G V$, Ganushchak Y, Geskes GG, Soemers AC, Maessen JG. Intracatdiac minipump provides significantly effective cardiac and circulatory support during severe acute cardiogenic shock. Eur I Heart Failure Suppl 2003; 2:145

Reesink KD, Dekker $A L$, van der Nagel $T$, Blom JA, Soemers AC, Geskes GG, Maessen JG, van der Veen FH. Physiologic Insensitive Left Ventricular Assist may Predispose Right-sided Circulatory Failure: A Pilot Simulation and Validation Study. Cardiovascular Engineering 2003; 8:33-34

Sauren LD, Reesink KD, Dekker AI, van der Nagel T, Severdija E, Soemers AC, Geskes GG, Maessen JG, van der Veen FH. Pulsatile extracorporeal life support may provide versatility in optimizing cardiac and circulatory support. Cardiovascular Engineering 2003; $8: 49$ 